\title{
The role of E3 ubiquitin ligase FBXO31-SCF in neuronal morphogenesis
}

\author{
Ph.D. Thesis \\ in the partial fulfilment of the requirements \\ for the degree "Doctor of Natural Sciences (Dr. rer. nat.)" \\ in the Neuroscience Program \\ at the Georg August University Göttingen, \\ Faculty of Biology
}

submitted by

Mayur Vadhvani

born in

Katihar, India

Göttingen 2012

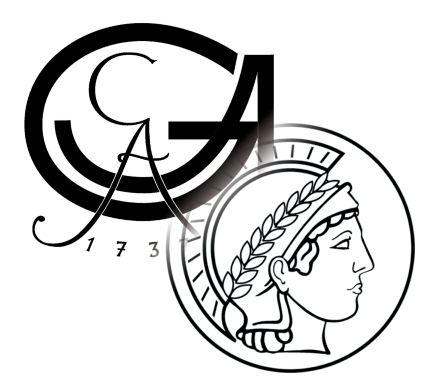


Members of the Thesis Committee:

Dr. Judith Stegmüller, Reviewer

Max Planck Institute of Experimental Medicine

Cellular and Molecular Neurobiology Group, University of Göttingen

Prof. Klaus-Armin Nave, Reviewer

Max Planck Institute of Experimental Medicine

Department of Neurogenetics, University of Göttingen

Dr. Till Marquardt

European Neuroscience Institute

Developmental Neurobiology Laboratory, University of Göttingen

Date of the oral examination: $\quad 24^{\text {th }}$ October, 2012 


\section{Affidavit}

I hereby declare that this $\mathrm{PhD}$ thesis "The role of E3 ubiquitin ligase FBXO31SCF in neuronal morphogenesis" has been written independently with no other aids or sources than quoted.

Mayur Vadhvani

August, 2012

Göttingen, Germany 


\section{Contents}

Contents

Acknowledgements viii

Abstract $\quad x$

Abbreviations $\quad$ xi

List of Figures . . . . . . . . . . . . . . . . . . xv

List of Tables . . . . . . . . . . . . . . . . . . . xviii

1 Introduction $\quad 1$

1.1 Neuronal development . . . . . . . . . . . . . . . . . . . . 1

1.1.1 Progenitor proliferation and differentiation . . . . . . . . . 2

1.1.2 Neuronal migration . . . . . . . . . . . . . . . . . 2

1.1.3 Neuronal polarity . . . . . . . . . . . . . . . . . . . . 3

1.1.4 Axon/Dendrite growth and guidance . . . . . . . . . . 6

1.2 Ubiquitin Proteasome System (UPS) . . . . . . . . . . . . 7

1.3 Anaphase Promoting Complex (APC) . . . . . . . . . . . . . . . 11

1.3.1 APC in neuronal development . . . . . . . . . . . . . . . 11

1.4 Skp1-Cullin1-F-box protein (SCF) complex . . . . . . . . . . . 15

1.4.1 SCF complex in neuronal development . . . . . . . . . . 16

1.4.2 SCF complex in neurodegenerative disorders . . . . . . . . . 20

1.5 Aim of the study . . . . . . . . . . . . . . . . . . . 22

2 Materials and Methods $\quad 24$ 


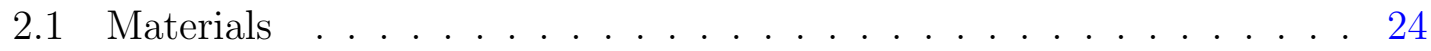

2.1.1 Antibodies ...................... . . 24

2.1.2 Chemicals, Enzymes and Kits . . . . . . . . . . . 24

2.1.3 Mammalian cell lines and bacterial strains . . . . . . . . . 28

2.1.4 Softwares used in the study . . . . . . . . . . . . . . 29

2.2 Methods . . . . . . . . . . . . . . . . . . . . . 29

2.2.1 Molecular biology and biochemical methods . . . . . . . . 29

2.2 .2 RNA interference . . . . . . . . . . . . . . . 31

2.2 .3 Site-directed mutagenesis . . . . . . . . . . . 33

2.2.4 Quantitative analysis of gene expression ......... 34

2.2.5 Biochemical assays ................... 34

2.2.6 Cell culture . . . . . . . . . . . . . . . . . . . . . . . 37

2.2.7 Transfection of primary and secondary cells . . . . . . . . 39

2.2.8 Immunocytochemistry . . . . . . . . . . . . . 40

2.2.9 Survival assay for cerebellar granule neurons . . . . . . . . . . 41

2.2 .10 In vivo electroporation . . . . . . . . . . . . . . . . . . . 41

2.2.11 Immunohistochemistry . . . . . . . . . . . . . . . 42

2.2.12 Morphometry . . . . . . . . . . . . . . 43

2.2 .13 Statistical tests . . . . . . . . . . . . . . 43

$\begin{array}{lll}3 & \text { Results } & 44\end{array}$

3.1 FBXO31 is a centrosomal E3 ubiquitin ligase . . . . . . . . . . . 44

3.1.1 Widespread expression of $F B X$ genes . . . . . . . . . . . . . 44

3.1.2 FBXO31 is brain-enriched F-box protein . . . . . . . . . . 44

3.1.3 FBXO31 is localized at the centrosome . . . . . . . . 46

3.2 Functional characterization of F-box protein FBXO31 in the nervous system . . . . . . . . . . . . . . . . . . . . . . 4 49

3.2.1 FBXO31 loss-of-function inhibits axon and dendrite growth in cerebellar granule neurons . . . . . . . . . . . . . . . . . 49 
3.2.2 FBXO31 gain-of-function promotes axon and dendrite growth in cerebellar granule neurons . . . . . . . . . . . . 56

3.2.3 FBXO31 loss-of-function reduces axon and dendrite growth in hippocampal and cortical neurons . . . . . . . . . . . 60

3.2.4 FBXO31 regulates the establishment of axon-dendrite polarity in neurons . . . . . . . . . . . . . . . . . 6 60

3.2.4.1 FBXO31 overexpression leads to a polarization defect in cerebellar granule neurons . . . . . . . . . . 60

3.2.4.2 FBXO31 regulates neuronal polarity in hippocampal neurons ...................... 62

3.2.5 FBXO31 promotes dendrite growth and neuronal migration in developing cerebellum . . . . . . . . . . . . 63

3.3 Par6c is a substrate of FBXO31-SCF in control of axon growth . . . . 68

3.3.1 FBXO31 interacts with Par/aPKC complex . . . . . . . 68

3.3.1.1 Par6c but not $\mathrm{PKC} \zeta$ is degraded by the ubiquitin proteasome system in granule neurons . . . . . . . 73

3.3.1.2 FBXO31 regulates the stability of Par6c . . . . . . 74

3.3.1.3 Par6c is polyubiquitinated and targeted for proteasomal degradation by FBXO31 . . . . . . . . . 75

3.3.2 Par6c acts as a suppressor of axon growth but not dendrite

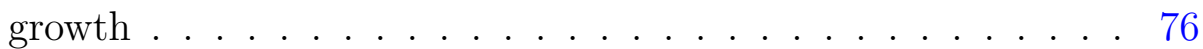

3.3.2.1 Par6c gain-of-function suppresses axon growth but not dendrite growth in neurons . . . . . . . . . 77

3.3.2.2 Par6c loss-of-function promotes axon but not dendrite growth . . . . . . . . . . . . . 79

3.3.3 Par6c acts downstream of FBXO31 in control of axon but not dendrite growth . . . . . . . . . . . . . . 83

3.4 FBXO31 interacts with Cdh1-APC in control of axon growth . . . . . 83

3.4.1 FBXO31 interacts with Cdh1 . . . . . . . . . . . 85 
3.4.2 Cdh1 regulates the stability of FBXO31 f . . . . . . . 86

3.4.3 FBXO31 acts downstream of Cdh1 in control of axon growth . 87

4 Discussion

4.1 Role of FBXO31 in neuronal morphogenesis . . . . . . . . . . . . . . 89

4.1.1 FBXO31 is a centrosomal E3 ubiquitin ligase . . . . . . . . . 89

4.1.2 FBXO31-SCF promotes of axon and dendrite growth in neurons 91

4.1.3 FBXO31-SCF regulates neuronal polarity . . . . . . . . . . . 92

4.1.4 FBXO31-SCF is essential for neuronal migration in the devel-

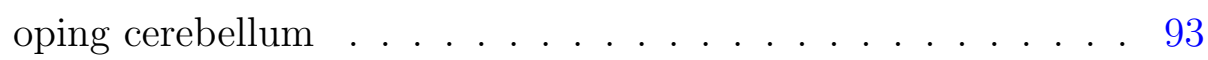

4.2 Insights into FBXO31-SCF-regulated neuronal morphogenesis . . . . 94

4.2.1 Par6 is a novel substrate of FBXO31-SCF in control of axon growth ............................ 94

4.2.2 FBXO31 is a novel interaction partner of E3 ubiquitin ligase Cdh1-APC . . . . . . . . . . . . . . 98 


\section{Acknowledgements}

As I walk 'down the memory lane' to write this important part of my thesis, I am overwhelmed to find an unending list of people who have supported me and walked with me during my journey in the last few years.

First of all, a sincere thanks to my supervisor Judith for her support and her trust in my abilities. I could enjoy my work in the lab as she encouraged me to try some 'random' experiments for science is not fun without them. I would also like to thank her for the lively discussions we had over science and research life. Thank you Judith, its been a pleasure working with you.

I thank my thesis committee members Prof. Klaus-Armin Nave and Dr. Till Marquardt for their advice and for critically evaluating my project during the scheduled meetings.

I am grateful to Prof. Michael Hörner, Sandra Drube and the Neuroscience program for their support and excellent environment they created for the PhD students.

The life in the lab would have been dull without the wonderful labmates I had. Thank you Nicola, David, Chaitali, Lidija and Shan for your help with the project. I enjoyed the scientific and non-scientific discussions with all of you. I am also grateful to Anna, Annika, Madhu, Shih-Ju and Siv (also for proof-reading my thesis) for creating a delightful lab environment and for withstanding my jokes now and then.

I also thank Dr. Hiroshi Kawabe for his discussion over the project and also for providing reagents to try some experiments. 
During my stay at Göttingen, I met many people who influenced me scientifically and personally. Thank you all for your support whenever it was needed. A sincere thanks to all my friends beyond Göttingen for their love and affection.

Last but not the least, I would like to thank my family - di, sunny, ma, papa and others - for trusting my abilities, for the faith they have in me and for everything they have done for me. I will cherish your love...forever! 


\section{Abstract}

Neuronal development is coordinated by the interplay of extrinsic cues and intrinsic factors. These extrinsic cues act through multiple intracellular signaling pathways to regulate the cytoskeleton machinery of the neuron that is essential during neuronal morphogenesis. Recent evidence identifies the ubiquitin proteasome system (UPS) as a crucial cell-intrinsic regulator of neuronal development. The Skp1-Cullin1-F-box protein (SCF) E3 ubiquitin ligase and in particular the substrate-recruiting adaptor subunit F-box proteins have emerged as essential modulators of diverse aspects of neuronal development including progenitor proliferation, migration, axon and dendrite growth and synaptogenesis. In this study, I identified the brain-enriched centrosomal F-box protein FBXO31-SCF as a novel regulator of neuronal morphogenesis both in vitro and in the developing cerebellum. While my study identifies FBXO31-SCF as a regulator of axonal identity, I also find that FBXO31-SCF promotes of axon and dendrite growth in neurons. To gain mechanistic insight into the FBXO31-regulated phenotypes, I uncovered the polarity protein Par6c as a novel interaction partner and a bona fide substrate of FBXO31. Further analysis revealed that FBXO31-SCF acts upstream of polarity complex protein Par6c to regulate axon growth but not dendrite growth in neurons. Taken together, my study gives a systematic insight into FBXO31-regulated events in developing neurons and thus introduces the E3 ubiquitin ligase FBXO31-SCF as a key regulator of neuronal development. 


\section{Abbreviations}

aa Amino acid(s)

AIS Axon initial segment

ANOVA Analysis of variance

aPKC Atypical protein kinase $\mathrm{C}$

APC Anaphase promoting complex

APP Amyloid precursor protein

APS Ammonium persulfate

ATP Adenosine triphosphate

BDNF Brain-derived neurotrophic factor

bHLH Basic helix loop helix

BME Basal medium eagle

BSA Bovine serum albumin

${ }^{\circ} \mathrm{C}$ degree Celcius/Centigrade

Cdc20 Cell division cycle protein 20

Cdc42 Cell division cycle protein 42

Cdh1 Cell division cycle 20 homologue 1

cDNA complementary deoxyribonucleic acid

CGN Cerebellar granule neuron

CNS Central nervous system

CRIB Cdc42/Rac interaction-binding

D-box Destruction-box

DBM Destruction-box mutant

DIV Days in vitro 
DLL Delta-like

DMEM Dulbecco's modified eagle's medium

DMSO Dimethyl sulfoxide

DNA Deoxyribonucleic acid

DTT Dithiotheritol

E.coli Escherichia coli

EDTA Ethylenediaminetetraacetic acid

FBX F-box protein

FCS Fetal calf serum

FGF Fibroblast growth factor

GFP Green fluorescent protein

GLR1 Glutamate receptor subunit 1

GSK3 $\beta \quad$ Glycogen synthase kinase 3 beta

GTPase guanosine $5^{\prime}$-triphosphate hydrolase

HBSS Hank's Balanced Salt Solution

HECT Homologous to E6AP C-terminus

HEPES 4-(2-Hydroxylethyl)piperazine-1-ethanesulfonic acid

hrs hours

HRP Horseradish peroxidase

HS Horse serum

IPC Intermediate precursor cell

IPTG Isopropyl $\beta$-D-thiogalactopyranoside

$\mathrm{kDa} \quad$ kilodalton

L-LTP Late long term potentiation

MDa Megadalton

$\min \quad$ Minute(s)

$\mathrm{mL} \quad$ milliliter

$\mathrm{mM} \quad$ millimolar

mRNA messenger ribonucleic acid

ms millisecond

$\mu \mathrm{g} \quad$ microgram 
$\mu \mathrm{L} \quad$ microliter

$\mu \mathrm{m} \quad$ micrometer

$\mu \mathrm{M} \quad$ micromolar

NEM N-ethylmaleimide

NCAM Neural cell adhesion molecule

NICD Notch intracellular domain

NGF Nerve growth factor

PAGE Polyacriamide gel electrophoresis

PB1 Phox/Bem1

PBS Phosphate buffer saline

PCR Polymerase chain reaction

PFA Paraformaldehyde

PI3K Phosphoinositide 3-kinase

PLL Poly-L-lysine

PTSD Post-traumatic stress disorder

REST Repressor element 1 silencing transcription factor

RING Really Interesting New Gene

RGC Radial glial cell

RNAi RNA interference

rpm Rotations per minute

RT Room Temperature

SCF Skp1-Cullin-F-box protein

SD Standard deviation

SDS Sodium dodecyl sulphate

sec Second(s)

SEM Standard error of mean

TEMED $\quad \mathrm{N}^{\prime}, \mathrm{N}^{\prime}, \mathrm{N}^{\prime}, \mathrm{N}^{\prime}$-tetramethylethylenediamine

TGF $\beta \quad$ Transforming growth factor beta

UPS Ubiquitin proteasome system

WT Wild type 


\begin{tabular}{|c|c|c|}
\hline Amino acids & Abbreviation & Single letter code \\
\hline Alanine & Ala & $\mathrm{A}$ \\
\hline Arginine & Arg & $\mathrm{R}$ \\
\hline Asparagine & Asn & $\mathrm{N}$ \\
\hline Aspartic Acid/Aspartate & Asp & $\mathrm{D}$ \\
\hline Cysteine & Cys & $\mathrm{C}$ \\
\hline Glutamine & Gln & $\mathrm{Q}$ \\
\hline Glutamic Acid/Glutamate & Glu & $\mathrm{E}$ \\
\hline Glycine & Gly & G \\
\hline Histidine & His & $\mathrm{H}$ \\
\hline Isoleucine & Ile & $\mathrm{I}$ \\
\hline Leucine & Leu & $\mathrm{L}$ \\
\hline Lysine & Lys & $\mathrm{K}$ \\
\hline Methionine & Met & M \\
\hline Phenylalanine & Phe & $\mathrm{F}$ \\
\hline Proline & Pro & $\mathrm{P}$ \\
\hline Serine & Ser & S \\
\hline Threonine & Thr & $\mathrm{T}$ \\
\hline Valine & Val & $\mathrm{V}$ \\
\hline Tryptophan & $\operatorname{Trp}$ & $\mathrm{W}$ \\
\hline Tyrosine & Tyr & $\mathrm{Y}$ \\
\hline
\end{tabular}

Nucleotide Single letter code

\begin{tabular}{ll}
\hline Adenine & A \\
Cytosine & C \\
Guanine & G \\
Thymine & T \\
Uracil & U \\
\hline
\end{tabular}




\section{List of Figures}

1.1 Stages of neuronal polarization in vitro . . . . . . . . . . . . . . 4

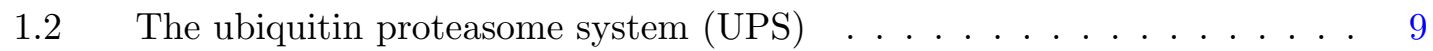

1.3 The anaphase promoting complex $(\mathrm{APC}) \ldots \ldots \ldots \ldots$

1.4 The Skp1-Cul1-F-box protein $(\mathrm{SCF})$ complex . . . . . . . . . . . . . 16

3.1 Expression of various $F B X$ genes in tissues of adult mouse . . . . . . . 45

3.2 Quantitative PCR analysis of FBXO31 expression in rat tissues . . . . . 46

$3.3 \quad$ FBXO31 is localized at the centrosome . . . . . . . . . . . . . 47

$3.4 \quad$ Validation of FBXO31 antibody . . . . . . . . . . . . . . . . . 48

$3.5 \quad$ FBXO31 deletion mutants and their localization . . . . . . . . . . 49

3.6 60-274 aa region is required for centrosomal localization of FBXO31 . . . 50

3.7 Targeting regions of FBXO31 short-hairpin RNAs . . . . . . . . . . 51

$3.8 \quad$ Validation of FBXO31 RNAi constructs . . . . . . . . . . . . . 52

$3.9 \quad$ FBXO31 knockdown reduces axon and dendrite growth in cerebellar

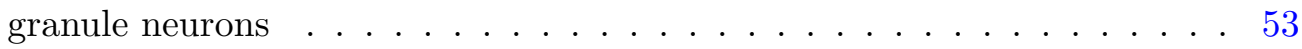

3.10 Generation and validation of FBXO31-Res plasmid . . . . . . . . . 54

3.11 FBXO31-Res reverses the FBXO31 RNAi phenotype on axon and dendrite growth in cerebellar granule neurons . . . . . . . . . . . . . 55

3.12 FBXO31 regulates neuronal survival . . . . . . . . . . . . . . . 56

3.13 FBXO31 overexpression promotes axon and dendrite growth in cerebellar granule neurons . . . . . . . . . . . . . . . . . . 5 57

3.14 FBXO31 $\Delta \mathrm{F}$ does not form a functional SCF complex . . . . . . . . 58

3.15 FBXO31-SCF ligase activity is essential for axon and dendrite growth in cerebellar granule neurons . . . . . . . . . . . . . . . . . . . . 59 
3.16 FBXO31 knockdown reduces axon and dendrite growth in hippocampal and cortical neurons . . . . . . . . . . . . . . . . . . 61

3.17 FBXO31 regulates neuronal polarization in cerebellar granule neurons . . 62

3.18 FBXO31 gain-of-function promotes multiple axon-phenotype in hippocampal neurons . . . . . . . . . . . . . . . . . . . . . 64

3.19 FBXO31 knockdown induces no-axon phenotype in hippocampal neurons 65

3.20 FBXO31 knockdown decreases dendrite growth of cerebellar granule neurons in vivo . . . . . . . . . . . . . . . . . . . . . 66

3.21 FBXO31 promotes neuronal migration of cerebellar granule neurons in developing cerebellumin vivo . . . . . . . . . . . . . . . . . . 67

$3.22 \quad$ FBXO31 interacts with Par6c . . . . . . . . . . . . . . . . 69

3.23 Par6c-PDZ domain interacts with FBXO31 . . . . . . . . . . . 70

3.24 Par6c and Par6b share a high sequence similarity in their PDZ domains . 70

3.25 FBXO31 interacts with Par6b . . . . . . . . . . . . . . . 71

3.26 FBXO31 interacts with $\mathrm{PKC} \iota$ and $\mathrm{PKC} \zeta \ldots \ldots . \ldots . \ldots . \ldots 72$

3.27 FBXO31 does not interact with Par3b . . . . . . . . . . . . . . 72

3.28 Par6c but not $\mathrm{PKC} \zeta$ is degraded in a proteasome-dependent manner in cerebellar granule neurons . . . . . . . . . . . . . . . . . . . 73

3.29 FBXO31 regulates the stability of Par6c . . . . . . . . . . . . . . 74

3.30 Par6c is polyubiquitinated by FBXO31-SCF $\ldots \ldots \ldots$. . . . . 76

3.31 Par6c polyubiquitination by FBXO31-SCF is K48-linked and not K63linked . . . . . . . . . . . . . . . . . . . . 77

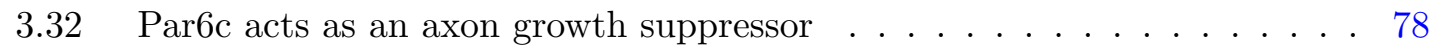

3.33 Validation of Par6c RNAi . . . . . . . . . . . . . . . . . . . 79

3.34 Par6c loss-of-function promotes axon but not dendrite growth . . . . . . 80

3.35 Generation and validation of Par6c-Res construct . . . . . . . . . . 81

3.36 Par6c-Res reverses the Par6c knockdown phenotype in axon growth of cerebellar granule neurons . . . . . . . . . . . . . . . . . . . . 82

3.37 Par6c acts downstream of FBXO31-SCF in axon but not dendrite growth

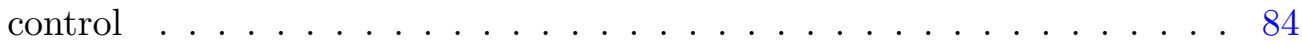

3.38 D-box motifs and their mutants in FBXO31 . . . . . . . . . . 85 
3.39 FBXO31 interacts with Cdh1 . . . . . . . . . . . . 86

3.40 Cdh1 interacts with FBXO31 D-box mutants . . . . . . . . . . . 86

3.41 Cdh1 regulates stability of FBXO31 . . . . . . . . . . . . 87

3.42 FBXO31 acts downstream of Cdh1 in control of axon growth in cerebellar granule neurons . . . . . . . . . . . . . . . 88

4.1 FBXO31-SCF promotes axon and dendrite growth in neurons . . . . . 98 


\section{List of Tables}

1.1 APC activators Cdh1 and Cdc20 in neuronal development . . . . . . . . . 14

$1.2 \quad$ F-box proteins in neuronal development . . . . . . . . . . . . . . . 20

$1.3 \quad$ F-box proteins in neurodegenerative diseases . . . . . . . . . . . . . . 22

2.1 Antibodies used in this study . . . . . . . . . . . . . . 25

2.2 Buffers and solutions used in the study . . . . . . . . . . . 26

2.3 Enzymes used in this study . . . . . . . . . . . . . . . . . . 27

$2.4 \quad$ Commercial kits used in this study . . . . . . . . . . . . . . . . . . 28

2.5 Cell lines and bacterial strains employed in the study . . . . . . . . . 28

$2.6 \quad$ List of softwares used in the study . . . . . . . . . . . . . . . . . . 29

$2.7 \quad$ short-hairpin RNA used in the study . . . . . . . . . . . . . . . 31

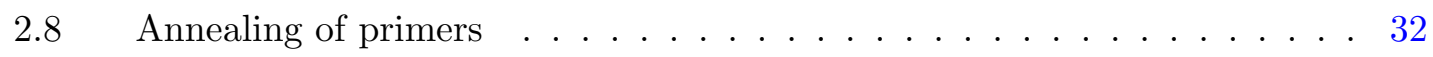

2.9 Site-directed mutagenesis PCR . . . . . . . . . . . . . . . 33

2.10 Primers for quantitative PCR . . . . . . . . . . . . . . . 34

A.1 List of plasmids used in the study . . . . . . . . . . . . . . . . . . 119

A.2 List of primers used for FBX gene expression study . . . . . . . . . . . 120

A.3 List of primers for site-directed mutagenesis . . . . . . . . . . . . . . 122

A.4 List of primers used for cloning . . . . . . . . . . . . . . . 123 
Carl Sagan

\section{Introduction}

\subsection{Neuronal development}

The mammalian brain is composed of billions of neurons and glial cells that act together in a fine-tuned network. Neurons are highly specialized cells with a polarized morphology consisting of two structurally and functionally distinct domains - axon and dendrites. Neurons make synaptic connections to relay the information. This flow of information occurs unidirectionally from dendrite to cell body to axon. Here, synaptic inputs from the connected neurons are integrated by dendrites, resulting in generation of an action potential at the cell soma. This is further propagated along the axon to the target cells. While neurons play an important role in information transfer, glial cells provide a supporting framework for neurons. Both neurons and glia are generated from a common pool of precursor cells in the developing brain. These neural precursor cells proliferate by symmetric division and subsequently undergo asymmetric division to generate neurons and glia. During neuronal development, neurons undergo dramatic changes in their morphology. Immature neurons derived from the precursor cells migrate over long distances to reach their final target. During the process of migration, neurons become polarized with distinct leading and trailing processes that eventually differentiate into dendrites and axon, respectively. These processes are further refined and fine-tuned enabling the integration of an individual neuron into the functional network. The phases of neuronal development and morphogenesis are elaborated in the subsequent sections. 


\subsubsection{Progenitor proliferation and differentiation}

During early embryonic development, the neuroectoderm gives rise to the neural tissue. As a result of neural induction, precursors are generated from the ectoderm under the control of diffusible neural inducers including chordin, noggin, follistatin and cerberus (Lamb et al., 1993; Hemmati-Brivanlou et al., 1994; Sasai et al., 1995; Biben et al., 1998; Streit and Stern, 1999). These cells undergo elongation as neuroepithilial cells along the embryonic axis and form the neural tube, which later differentiates into brain and spinal cord.

As neurogenesis proceeds, neuroepithilial cells undergo massive proliferation to generate more precursor cells by symmetric divisions. These progenitors are polarized into apical and basal domains with their nuclei undergoing interkinetic movement along the apico-basal axis during cell division (Götz and Huttner, 2005). Neuroepithilial progenitors give rise to radial glial cells (RGCs). Recently, it has been shown that the RGCs are the major source of neurons during development (Malatesta et al., 2000; Noctor et al., 2001, 2002; Anthony et al., 2004). RGCs span the width of the entire developing cortex with their soma remaining close to the apical/ventricular surface and a long radial process maintaining contact with the basement membrane of the pial surface (Cameron and Rakic, 1991). These divide asymmetrically to generate another RGC and an intermediate precursor cell (IPC) or a neuron. These IPC undergo another round of symmetric division to generate two neurons. These immature neurons then migrate along the processes of the radial glial cell to reach their final destination (Fishell and Kriegstein, 2003).

\subsubsection{Neuronal migration}

Coordinated neuronal migration is a major hallmark of the developing brain. Newly generated neurons migrate from ventricular zone to their final position under the influence of secreted guidance cues. In the developing cortex, the neurons migrate along radial glial cells from the sub-ventricular zone towards the cortical plate 
in an inside-out manner with newly arriving cells migrating radially past the existing neurons. In contrast, in the developing cerebellum, granule neurons migrate from the germinal zone in the external granule layer towards the internal granule layer (Hatten, 1999). The migration of neurons is achieved by the extension of cellular protrusions in the direction of migration referred to as the leading process, followed by nuclear movement in the direction of migration. This glial-guided neuronal migration is mediated by several cell-adhesion molecules such as integrins, neuregulins and astrotactins (Fishell and Hatten, 1991; Anton et al., 1997, 1999; Solecki, 2012).

Besides the cell-adhesion molecules, neuronal migration is also regulated by several extrinsic cues including growth factors such as NGF (nerve growth factor), BDNF (brain-derived neurotrophic factor), FGF (fibroblast growth factor) and secreted molecules such as slits, netrins, semaphorins and reelin (Marin, 2003; Casazza et al., 2007; Lai Wing Sun et al., 2011). At the marginal zone, the Cajal-Retzius cells secrete reelin that upon binding to its receptor activates downstream signaling cascade, which induces neurons to settle at their appropriate cortical layers (Soriano and Del Río, 2005). The reeler mouse mutants show a cortical lamination defect with the neurons aligned in an outside-in fashion (Hatten, 1999). As a result of consorted action of these secreted molecules, neurons migrate to their final destinations.

\subsubsection{Neuronal polarity}

Neuronal polarization is fundamental to formation of two structurally and functionally distinct domains in the neurons - axons and dendrites. Many studies have implicated various signaling cascades controlled by extracellular signals that lead to cytoskeletal remodeling and localization of specific proteins in one of the compartments. Cultured hippocampal neurons have been widely used to study the signaling cascades that guide neuronal polarization in vitro. These neurons display specific morphological characteristics that were first described by Dotti and colleagues (Dotti et al., 1988). At stage 1, neurons display intense lamellipodial and filopodial-protrusions, which leads to emergence of multiple neurites (Stage 2). 
Stage 3 represents a critical step when the neuronal symmetry breaks and one neurite grows rapidly to become the axon while other neurites acquire dendritic identity. Stage 4 is characterized by rapid growth of axon and dendrites, while stage 5 involves terminal differentiation of neurons and formation of dendritic spines (Craig and Banker, 1994; Barnes and Polleux, 2009) (Figure 1.1).

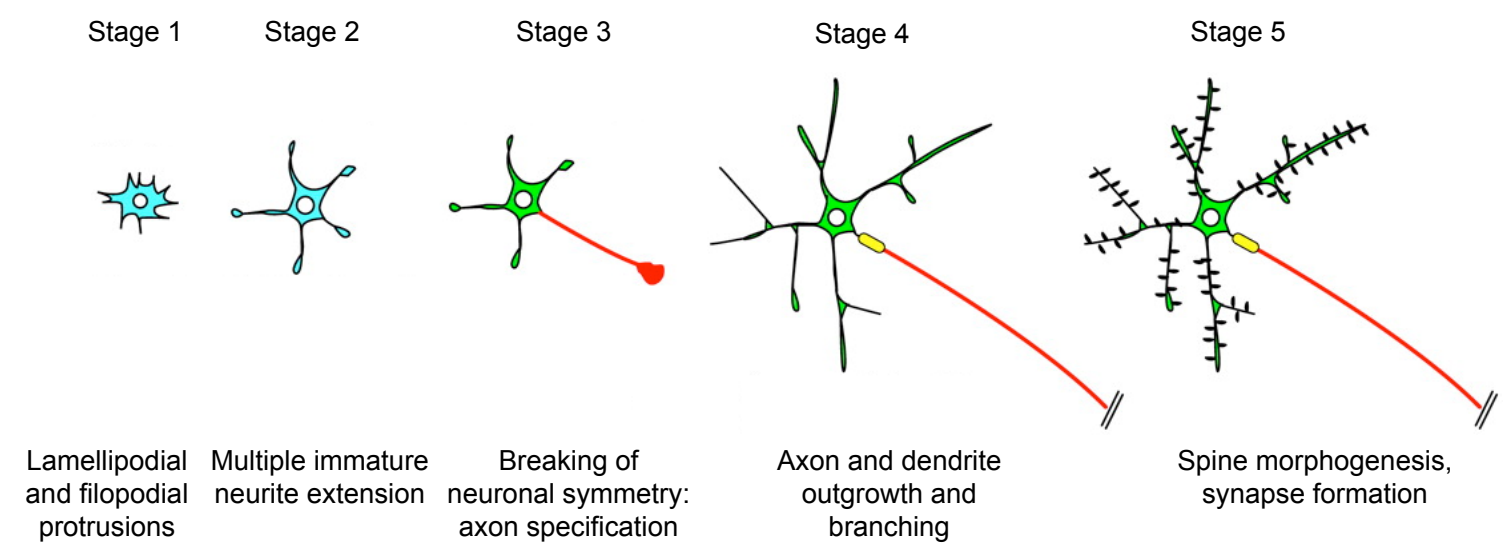

Figure 1.1: Stages of neuronal polarization in vitro. Schematic representation of neuronal polarization in vitro. Soon after plating, the neurons develop lamellipodial/filopodial protrusions (stage 1) that leads to emergence of several immature neurites (stage 2). At stage 3, neuronal symmetry breaks and one neurite grows rapidly to form the axon (red) whereas other neurites acquire dendritic identity. Stage 4 is characterized by rapid axon and dendrite growth. In stage 5 , the neurons are terminally differentiated with dendritic spines and synapses. Modified from (Polleux and Snider, 2010).

This morphological compartmentalization of neurons into distinct domains is governed by several extrinsic cues including growth factors and secreted molecules such as BDNF, neurotrophins, TGF $\beta$ and Wnt (Zhang et al., 2007; Yi et al., 2010; Cheng et al., 2011). The information from these cues is relayed via several intracellular signaling pathways that converge on the key mediators of neuronal polarity PI3K/GSK3 $\beta$ and par polarity complex (Arimura and Kaibuchi, 2007). While both these mediators lead to cytoskeletal remodeling, the par polarity complex has been well studied for its role in establishment of neuronal polarity. 
The par complex was first identified in a genetic screen based on asymmetric divisions occurring in the C. elegans zygotes (Kemphues et al., 1988). The study identified six par genes whose mutations showed an abnormal cleavage pattern and subsequent studies identified the molecular functions of these par proteins. Par1 and Par4 encode serine/threonine protein kinases (Morton et al., 1992; Guo and Kemphues, 1995). Par2 has a RING finger domain that may act in the ubiquitination pathway (Levitan et al., 1994). Par3 and Par6 contain PDZ domains suggesting their role as scaffolding proteins (Etemad-Moghadam et al., 1995; Watts et al., 1996). Par5 is a member of 14-3-3 family that binds to phosphorylated serines and threonines (Morton et al., 2002). Tabuse and colleagues identified the seventh member of the par gene family encoding atypical protein kinase $\mathrm{C}$ (PKC-3) (Tabuse et al., 1998). RNAi-induced knockdown of PKC-3 in the C. elegans embryos induced lethality including defects in early symmetric divisions that were similar to par-like phenotypes.

The Par/aPKC complex primarily comprises Par6 (Par6 $\alpha$, Par6 $\beta$ and Par6 $\gamma$ ), Par3 and aPKC (PKC $\iota$ and $\mathrm{PKC} \zeta)$. Par6 proteins are structurally similar and form a complex with Par3 and aPKC. They consist of three conserved domains: The N-terminal Phox/Bem1 (PB1) domain that binds to other PB1 domain containing proteins such as aPKC, the Cdc42/Rac interaction-binding (CRIB) motif that binds to $\mathrm{Cdc} 42$ or Rac GTPases in their active, GTP-bound state and the PDZ domain that binds to other proteins such as Par3. Aside from the PB1 domain, aPKCs harbour a catalytically active kinase domain at the C-terminus which mediates the phosphorylation of several proteins including Par3 (Assémat et al., 2008).

The function of the par protein complex in epithelial and neuronal cell polarity is highly conserved among species (Shin et al., 2006). The first evidence confirming the role of the par complex in neuronal polarity was illustrated in hippocampal neurons (Shi et al., 2003). Par6 is a predominant centrosomal protein and as neurons polarize, Par6 together with Par3 is selectively enriched at the tip of future axon (Solecki et al., 2004, 2009; Mori et al., 2009; Cheng et al., 2011). Disruption 
of the polarized distribution of either Par6 or Par3 impairs axon specification in hippocampal neurons. Since then, there has been mounting evidence that supports the role of the Par6/Par3/aPKC complex in neuronal polarity (Shi et al., 2004; Nishimura et al., 2005; Schwamborn et al., 2007; Vohra et al., 2007; Yi et al., 2010). The primary defect in neuronal polarization caused by disrupting the function of the par complex is the failure of neurites to differentiate into either axons or dendrites. Neuronal polarity defects manifest in various ways including no axon or dendrite formation, multiple axons or multiple dendrites with no axon.

\subsubsection{Axon/Dendrite growth and guidance}

Once neuronal polarity is established, the axonal process grows rapidly under the influence of extracellular factors that are required for gene transcription and cytoskeletal assembly (Lentz et al., 1999; Goldberg et al., 2002; Ozdinler and Macklis, 2006). Several extrinsic factors namely NGF, BDNF, FGF, NCAM (neural cell adhesion molecule) and N-cadherin have been well characterized for triggering several intracellular signaling pathways in axon growth control (Bixby and Harris, 1991; Zhou and Snider, 2006). Axon growth is simultaneously facilitated by its guidance to the target and integration into the functional circuitry. The axonal guidance and pathfinding is regulated by several guidance cues including netrins, slits, semaphorins, ephrins, hedgehog, Wnt and TGF $\beta$, which are highly conserved amongst various species (Tessier-Lavigne and Goodman, 1996; Araújo and Tear, 2003; Huber et al., 2003; Evans and Bashaw, 2010). These guidance cues act primarily on the growth cones of the axon. The leading edge of the axon growth cone is a motile structure that constantly undergoes remodeling and is enriched with the cytoskeletal protein actin (Dent and Gertler, 2003; Lowery and Van Vactor, 2009). Actin assembly is the key process that controls steering of the growth cones. While the attractants promote actin polymerization in the growth cones, the repellents decrease the polymerization of actin. Actin assembly is itself regulated by several RhoGTPases - Cdc42/Rac1/RhoA and their downstream effectors. 
Besides axons, dendrites also undergo constant growth and pruning in order to establish synaptic contacts with their target synapses. Dendrites are highly branched structures, that form depending on the neuronal cell type most elaborate arbors. The complex dendritic structure is also regulated by several extrinsic cues such as BDNF, notch, slits and Wnt that allow dendritic growth as well as retraction (Kim and Chiba, 2004; Dijkhuizen and Ghosh, 2005). In addition, the growth and refinement of the dendritic tree are strongly influenced by synaptic activity and calcium signaling (Zhang and Poo, 2001). As dendrites mature, they form functional synapses with their partner axon. Thus, appropriate morphogenesis of individual neurons at different stages of development is crucial for their integration into the functional network.

\section{$1.2 \quad$ Ubiquitin Proteasome System (UPS)}

As introduced in the previous sections, extrinsic cues including growth factors, guidance proteins and other extracellular cues regulate the overall design of the neuronal shape as well as fine structural elements by mediating cellular responses that directly influence cytoskeletal dynamics. Accumulating evidence also supports the role of cell-intrinsic mechanisms in neuronal morphogenesis (Frank and Tsai, 2009; de la Torre-Ubieta and Bonni, 2011). These mechanisms are primarily inherited pathways that operate largely independent of the cellular environment and orchestrate neuronal responses to extrinsic cues. The regulation of gene expression by transcription factors represents a major mode of cell-intrinsic control of neuronal morphogenesis (Chédotal and Rijli, 2009; Moore et al., 2011). Transcription factors govern entire sets of developmental programs by inducing or repressing gene expression both spatially and temporally. Besides transcription factors, the ubiquitin proteasome system has recently emerged as an important cell-intrinsic regulator of neuronal morphogenesis (Frank and Tsai, 2009; Yang et al., 2010). 
The ubiquitin proteasome system is the major pathway for the control of intracellular protein degradation, first described in rabbit reticulocytes by Hershko and colleagues (Hershko et al., 1979). This spatial and temporal control of protein degradation is fundamental to cellular physiology including cell cycle regulation, various developmental programs and responses to external cues. Protein degradation by the UPS occurs via sequential activity of three enzymes namely E1 ubiquitin-activating enzyme, E2 ubiquitin-conjugating enzyme and E3 ubiquitin ligase, eventually leading to ubiquitination of the target protein, which is recognized by the $26 \mathrm{~S}$ proteasome for degradation (Hershko and Ciechanover, 1998).

Ubiquitin is a highly conserved 76 amino acid protein that is activated for conjugation in an ATP-dependent manner by the E1 ubiquitin-activating enzyme, which catalyzes a thioester linkage between the C-terminal glycine residue in ubiquitin and a cysteine residue in the E1 ubiquitin-activating enzyme. The activated ubiquitin is linked to the E2 ubiquitin-conjugating enzyme by a transesterification reaction. Finally, a specific E3 ubiquitin ligase recruits the E2-Ub conjugate and the substrate to mediate the transfer of ubiquitin from the E2 to the substrate. Ubiquitin forms an isopeptide bond with the target lysine residue of the substrate (Hershko and Ciechanover, 1998) (Figure 1.2). Conjugation of ubiquitin chains can occur via different lysine residues in ubiquitin. Lysine 48-linked polyubiquitination is often associated with proteasomal degradation, while lysine 63-linked polyubiquitin chains represent a non-proteolytic modification of proteins. Ubiquitin can also be conjugated through other lysine residues including Lys6, Lys11, Lys27, Lys29 and Lys33 (Ikeda and Dikic, 2008). Although all lysine residues in ubiquitin have been shown to participate in chain formation, only a few studies have addressed their biological significance. Whereas Lys63-linked polyubiquitin chain formation is involved in DNA repair and signal transduction (Spence et al., 1995; Kerscher et al., 2006), Lys11 and Lys63 mixed linkage chains function as a signal for internalization of MHC I (major histocompatibility complex class I) through its interaction with epsin 1 (Goto et al., 2010). 


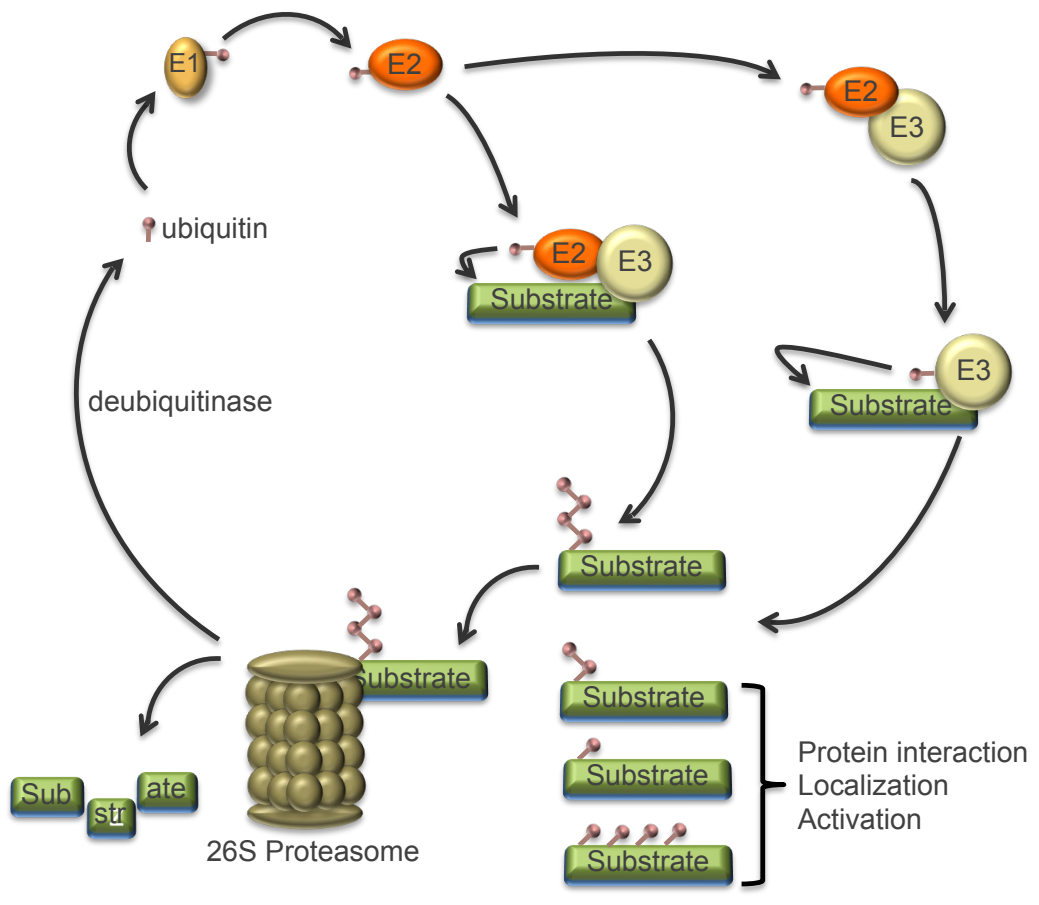

Figure 1.2: The ubiquitin proteasome system (UPS). Ubiquitin is activated by $\mathrm{E} 1$ ubiquitinactivating enzyme in an ATP-dependent manner. Activated ubiquitin is then linked to the E2 ubiquitin-conjugating enzyme that associates with the specific E3 ubiquitin ligase. Activated ubiquitin is transferred from E2 conjugating enzyme to the substrate either directly or through an E3 ubiquitin ligase resulting in either mono-, di- or polyubiquitination of the substrate. While polyubiquitinated substrates are directed to the $26 \mathrm{~S}$ proteasome for degradation, mono- and diubiquitination affect protein interaction, localization and activation. Deubiquitinating enzymes replenish ubiquitin to the cellular pool of ubiquitin.

The most remarkable feature of the UPS is the large number of E3 ubiquitin ligases. With more than 600 E3 ubiquitin ligases encoded by the human genome, they outnumber the family of protein kinases (Cohen and Tcherpakov, 2010). Although E3 ligases confer substrate specificity, individual E3 ligases can recruit more than one substrate (Peters, 2006). Conversely, individual substrates may be targeted by more than one E3 ligase (Kanie et al., 2012). E3 ligases have been classified mainly into two families: the HECT domain E3 ligases and the RING domain E3 ligases.

The HECT (Homologous to the E6AP C-terminus) E3 ligases form a thiol ester intermediate with activated ubiquitin. The HECT domain (approximately 350 
amino acids) binds the E2-Ub intermediate and accepts ubiquitin at a conserved cysteine residue. This ubiquitin is then transferred to the substrate by covalent linkage. HECT E3 ligases are involved in cancer progression, cardiovascular and neurological disorders (Pickart and Eddins, 2004). For example, HECT E3 ligase NEDD4-1 (neuronal precursor cell expressed and developmentally downregulated protein 4-1) targets PTEN (Phosphatase and tensin homolog) and Rap2 (Wang et al., 2007; Kawabe et al., 2010). While NEDD4-1 mediates polyubiqutination and degradation of tumor suppressor PTEN to regulate tumorogenesis, it brings about ubiquitination of Rap2A to promote dendrite growth. The HECT E3 ligase Smurf1 controls cell polarity and axon growth by targeting Rho GTPase RhoA for degradation (Wang et al., 2003; Cheng et al., 2011). In addition to refinement of neuronal circuitry, the HECT ligases have been well-studied in context of neurodevelopmental disorders. A mutation in the Ube3a gene encoding E3 ubiquitin ligase E6AP is associated with Angelman syndrome characterized by developmental delays, intellectual disability and speech impairments (Mabb et al., 2011). E6AP has been implicated in synapse development and maintenance (Dindot et al., 2008; Greer et al., 2010; Margolis et al., 2010).

The RING (Really Interesting New Gene) E3 ligases are characterized by the presence of a RING domain that consists of a short motif rich in cysteine and histidine residues, which coordinate two zinc ions (Pickart and Eddins, 2004). These RING E3 ligases act as monomers or form a multimeric complex to mediate ubiquitination and degradation of the target protein. The multimeric RING ligases Anaphase Promoting Complex (APC) and Skp1-Cullin1-F-box protein (SCF) complex have been characterized primarily for their function in cell cycle progression and maintenance (Peters, 1998). Both APC and SCF are discussed in the subsequent sections. 


\subsection{Anaphase Promoting Complex (APC)}

$\mathrm{APC}$ is a $1.5 \mathrm{MDa}$ multimeric RING E3 ligase complex that is composed of at least 12 subunits including the cullin protein APC2 and the RING-finger protein APC11 (Peters, 2006) (Figure 1.3). APC2 and APC11 form the catalytic part of the substrate that can mediate ubiquitination in vitro with reduced substrate specificity (Tang et al., 2001). The APC activity is stimulated in a cell-cycle dependent manner upon binding to the activator proteins Cdc20 or Cdh1. While the association of APC with Cdc20 is required for its activity during early mitosis, Cdh1 associates with APC during late mitosis and G1 phase (Harper et al., 2002; Peters, 2002). Besides stimulating the activity of the core complex, Cdc20 and Cdh1 confer substrate specificity to the complex. For example, during early mitosis, Cdc20-APC targets cyclin B1 and securin for degradation to initiate metaphase-to-anaphase transition. Meanwhile, Cdh1-APC targets other APC substrates including mitotic cyclins and Cdc20 to exit the mitotic cycle. During G1, Cdh1 promotes degradation of the the F-box protein and SCF complex subunit Skp2 to prevent premature entry into the S-phase (Hu et al., 2011).

Both APC coactivators - Cdh1 and Cdc20 - contain a C-terminal WD40 domain that mediates the interaction of APC to the substrates. Cdh1 and Cdc20 mediate recognition and recruitment of substrates harboring D-box (destruction box) or KEN box recognition motifs (Peters, 2006). While most substrates harboring these degrons are recruited to the APC complex for ubiquitination, some proteins harboring these degrons are not degraded by APC. For example, although Emi1 harbors a D-box domain, it acts as a pseudosubstrate of Cdh1 and inhibits APC activity by competing with other Cdh1 substrates (Miller et al., 2006).

\subsubsection{APC in neuronal development}

In 1999, Gieffers and colleagues described the expression and ligase activity of Cdh1, a crucial cell cycle regulator, in the post-mitotic neurons (Gieffers et al., 


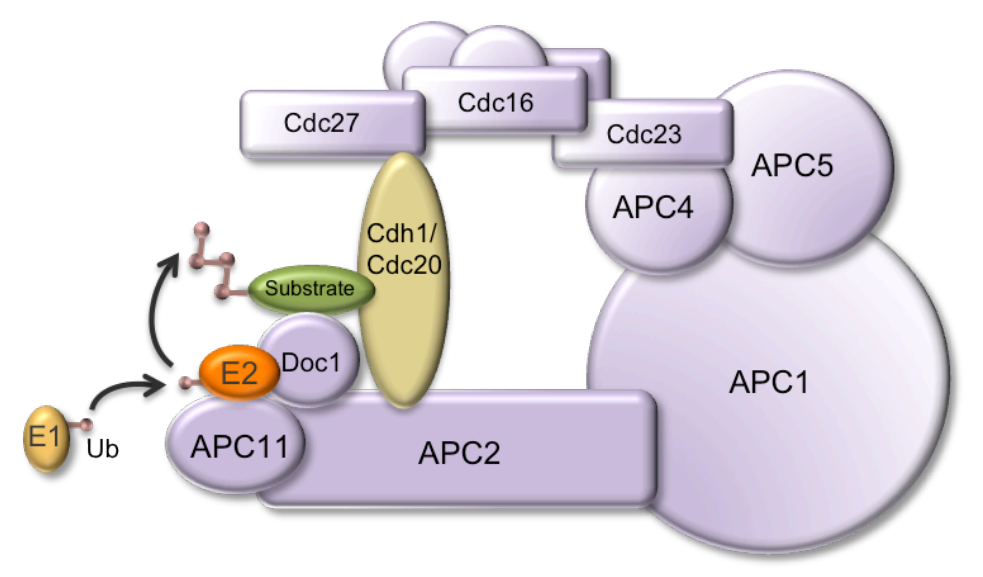

Figure 1.3: The anaphase promoting complex (APC). The APC complex consists of at least 12 different subunits including the scaffolding protein APC2 and the RING-finger protein APC11. The co-activators - Cdh1 or Cdc20 - bind to the substrates harboring D-box or KEN box motifs to recruit them to the APC core. Cdc27/APC3 binds to Cdh1 or Cdc20 and Doc1/APC10 is essential for substrate recognition and the processivity of the complex. The other subunits including Cdc16, Cdc23, APC1, APC4 and APC5 are involved in protein interactions. Modified from (Peters, 2006).

1999). Since then, several reports have attributed Cdh1 function in neuronal development (summarized in Table 1.1). Cdh1 has emerged as a prominent regulator of axon growth in neurons (Konishi et al., 2004). It suppresses axon growth by targeting SnoN, Id2 and Smurf1 for degradation (Lasorella et al., 2006; Stegmüller et al., 2006; Kannan et al., 2012). The degradation of SnoN by Cdh1-APC in neurons is regulated by the TGF $\beta$-Smad2 pathway. Accordingly, TGF $\beta$-Smad2 signaling restricts axon growth. In this context, SnoN, a negative regulator of TGF $\beta$ signaling, associates with transcriptional co-activator p300. Both SnoN and p300 are required for the regulation of Ccd1. Ccd1, an actin-binding protein, localizes to the axon terminals and loss-of-function studies suggest that Ccd1 mediates SnoN-dependent axon growth in granule neurons (Stegmüller et al., 2006, 2008; Ikeuchi et al., 2009). Simultaneously, Cdh1-APC activation results in degradation of another transcription factor Id2 to regulate axon growth (Lasorella et al., 2006). Id2 D-box mutant, which is resistant to Cdh1-APC-mediated degradation, enhances axon growth in granule neurons and overcomes the myelin inhibitory signals for growth. In the 
cytoplasm, Cdh1-APC targets the E3 ubiquitin ligase Smurf1 for degradation to suppress axon growth (Kannan et al., 2012). Smurf1 in turn promotes axon growth by targeting the small GTPase RhoA for degradation (Cheng et al., 2011).

Aside from its axon growth-suppressing function, Cdh1-APC also modulates synaptic strength and plasticity. Cdh1-APC regulates the expression of GluR1 subunit of the AMPA receptor by proteasomal degradation (Fu et al., 2011). Downregulation of GluR1 and reduction in glutamatergic transmission is mediated by Eph4Cdh1-APC-dependent signaling in response to prolonged activity at the synapse. In invertebrates, Fzr1, the Drosophila homologue of mammalian Cdh1, has also been shown to regulate synapse development by mediating ubiquitination of Lipirin- $\alpha$ (van Roessel et al., 2004). Lipirin- $\alpha$ interacts directly with Dlar, a receptor tyrosine phosphatase, to modulate synaptic bouton number, underscoring the role of Cdh1 in synapse formation.

Moreover, recent work by Silies and Klämbt has shown that glial migration in Drosophila along the axon is regulated by Cdh1-APC (Silies and Klämbt, 2010). They report that Drosophila Cdh1/Fzr is essential for establishment of graded axonal distribution of the immunoglobulin superfamily cell adhesion molecule Fasciclin2 (Fas2). In Drosophila motor neurons, Fas2 interacts homophilically with glial Fas2 and glial migration is initiated along axonal segments with lower levels of Fas2, but stalls in axonal domains with high levels of Fas2. Thus, Cdh1-APC has emerged as an important mediator in coordinating the migration of neuronal and glial cells during development.

An emerging role of Cdh1-APC is in learning and memory. Garcia-Higuera and colleagues found deficits in the neuromuscular coordination and memory in the Cdh1 conditional knockout ( $C d h 1^{+/-}$;Sox2-Cre) animals shown by the tightrope test and the novel-object recognition test, respectively (García-Higuera et al., 2008). Moreover, conventional Cdh1 heterozygous animals show impairment in late-phase long term potentiation (L-LTP) that is evoked by multiple high-frequency stimulation in Schaffer collateral-CA1 synapses. In concordance with these findings the 
animals show deficits in contextual fear memory, a hippocampus-dependent task (Li et al., 2008). The requirement of Cdh1-APC in higher cognitive functions is further supported by the memory deficits observed in the APC2 knockouts (Kuczera et al., 2011). These knockout animals show impairment in spatial memory and extinction of fear memories. Defective fear extinction, in particular, is a characteristic of anxiety disorders such as phobia or post-traumatic stress disorder (PTSD).

Table 1.1: APC activators Cdh1 and Cdc20 in neuronal development

\begin{tabular}{|c|c|c|c|}
\hline Activator & $\begin{array}{l}\text { Neuronal } \\
\text { substrate }\end{array}$ & Function & Reference \\
\hline \multirow[t]{10}{*}{ Cdh1 } & SnoN & Axon growth & (Stegmüller et al., 2006) \\
\hline & $\operatorname{Id} 2$ & Axon growth & (Lasorella et al., 2006) \\
\hline & Smurf1 & Axon growth & (Kannan et al., 2012) \\
\hline & Skp2 & Neuronal differentiation & (Harmey et al., 2009) \\
\hline & GluR1 & Synaptic plasticity & $\begin{array}{l}\text { (Juo and Kaplan, 2004; } \\
\text { Fu et al., 2011) }\end{array}$ \\
\hline & Cyclin B1 & Neuronal survival & (Almeida et al., 2005) \\
\hline & Lipirin- $\alpha$ & Synaptic size and activity & (van Roessel et al., 2004) \\
\hline & Cdk5 & Neuronal cell cycle & (Zhang et al., 2012) \\
\hline & $\begin{array}{l}\text { Fascicilin2 } \\
\text { (putative) }\end{array}$ & Glial migration & (Silies and Klämbt, 2010) \\
\hline & Pfkfb3 & Glycolysis & $\begin{array}{l}\text { (Herrero-Mendez et al., } \\
2009)\end{array}$ \\
\hline \multirow[t]{2}{*}{$\operatorname{Cdc} 20$} & $\mathrm{Id} 1$ & Dendrite growth & (Kim et al., 2009) \\
\hline & NeuroD & $\begin{array}{l}\text { Presynaptic } \\
\text { differentiation }\end{array}$ & (Yang et al., 2009) \\
\hline
\end{tabular}

Besides Cdh1, Cdc20-APC is also present in post-mitotic neurons, where it plays a role in dendritic morphogenesis (Kim et al., 2009; Puram et al., 2011). Knockdown of Cdc20 impairs the formation of dendritic arbors in granule neurons 
(Kim et al., 2009). Cdc20 is a centrosome-associated protein that is activated by interaction with HDAC6. This interaction of Cdc20-APC with HDAC6 drives the differentiation of dendrites in post-mitotic neurons. Phosphorylation of Cdc20-APC by CaMKII $\beta$ leads to its dissociation from the centrosome and to retraction of dendrites (Puram et al., 2011). Cdc20 also triggers the degradation of the transcription factor NeuroD2 to promote presynaptic differentiation (Yang et al., 2009). The NeuroD2 target gene Complexin2 encodes for a regulator of synaptic vesicle fusion and thus mediates the ability of NeuroD2 to suppress presynaptic differentiation (Huntwork and Littleton, 2007). In summary, both coactivators of the APC, Cdh1 and Cdc20, have emerged as important regulators of neuronal development.

\subsection{Skp1-Cullin1-F-box protein (SCF) complex}

The SCF complex is another multimeric RING E3 ligase that plays an essential role during cell cycle and is structurally related to the APC complex. This complex is composed of the scaffolding protein Cullin (Cul), S-phase kinase associated protein 1 (Skp1), the RING domain containing protein Rbx1/Roc1 and an interchangeable component, the F-box protein, that binds to Skp1 and Cul1 through its F-box domain (Zheng et al., 2002) (Figure 1.4). F-box proteins are responsible for substrate recognition. There are sixty-nine F-box proteins identified in humans that serve as adaptors of the SCF complex and thus provide the basis for a large number of substrate-specific ubiquitination pathways (Kipreos and Pagano, 2000).

F-box proteins have been classified into three subfamilies: those with WD40 domains (FBXWs), those with leucine-rich repeats (FBXLs) and those that harbor other domains (FBXOs) (Kipreos and Pagano, 2000; Jin et al., 2004). Notably, only a minority of the sixty-nine F-box proteins has been well-characterized. The beststudied F-box proteins are FBXW1 and FBXW11 (also referred to as $\beta$-TrCP1 and $\beta$-TrCP2, respectively), which act as key players in cell cycle regulation (Frescas and Pagano, 2008). Both FBXW1 and FBXW11 have similar substrate specificities and 


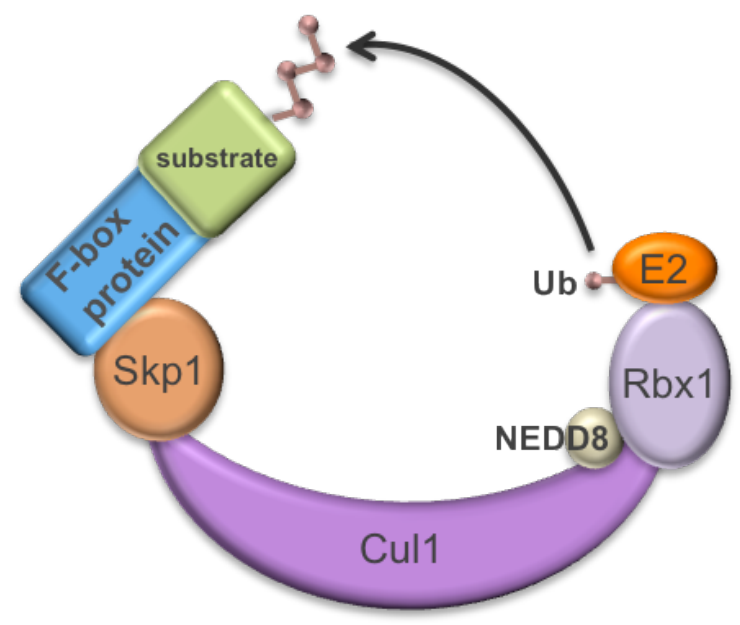

Figure 1.4: The Skp1-Cullin1-F-box protein (SCF) complex. The SCF complex consists of the scaffolding protein Cullin1, S-phase-associated protein kinase 1 (Skp1), RING finger protein $\mathrm{Rbx} 1$ and the adaptor protein F-box protein. F-box proteins bind to Skp1 and Cul1 through their F-box domains and recruit substrates for ubiquitination.

frequently function redundantly (Guardavaccaro et al., 2003; Shirogane et al., 2005). During cell cycle transition, $\beta$-TrCP acts as both positive and negative regulator of the cell cycle by inducing the degradation of Cdc25A (cell division cycle 25A), Wee1a and Emi1 (a pseudosubstrate of APC) (Frescas and Pagano, 2008). An alteration in FBXW1 or FBXW11 activity contributes to unrestrained proliferation and cancer (Branzei and Foiani, 2008). Another F-box protein, FBXL1 (also referred to as SKP2), also plays a crucial role in cell cycle progression where FBXL1 ubiquitinates the tumor suppressor p27 phosphorylated at Thr187 by Cdks (Tsvetkov et al., 1999). Besides regulating p27 levels, FBXL1 also mediates the degradation of several cell cycle and oncogenic proteins including cyclin E, cyclin D1, myc and BRCA2 (Frescas and Pagano, 2008).

\subsubsection{SCF complex in neuronal development}

As outlined earlier, the SCF complex has been largely studied in cell cycle regulation. The quest for neuronal F-box proteins has only recently begun and has 
revealed important functions of F-box proteins in the brain including neurogenesis, neuronal migration, axon growth, dendritic patterning and synaptogenesis (summarized in Table 1.2).

The F-box proteins FBXW1 and FBXW11 $(\beta$-TrCP1 and $\beta$-TrCP2, respectively), in agreement with their role in cell cycle, regulate proliferation and differentiation of neural progenitors. FBXW1 and FBXW11 interact with and facilitate degradation of $\beta$-catenin, a downstream mediator of Wnt signaling. In the canonical Wnt pathway, the binding of the ligand Wnt to its receptor Frizzled causes activation of Disheveled (Dvl) that in turn inhibits GSK3 $\beta$ activity. This results in translocation of $\beta$-catenin into the nucleus, where its binding to the LEF/TCF transcription complex activates Wnt responsive genes. In absence of Wnt, active GSK3 $\beta$ phosphorylates $\beta$-catenin that is recognized by $\beta$-TrCP for degradation (Hart et al., 1999; Latres et al., 1999). GSK3 $\beta$ activity is inhibited by DISC1 (disrupted in schizophrenia 1), which also plays a critical role in progenitor proliferation. This inhibition of GSK3 $\beta$ activity by DISC1 in the progenitor leads to stabilization of $\beta$-catenin that promotes proliferation (Mao et al., 2009). Besides regulating $\beta$-catenin stability, $\beta$-TrCP also mediates the degradation of REST (repressor element 1 silencing transcription factor) to promote neurogenesis. REST is a DNA-binding protein that recognizes motifs in the promoter of many neuronal genes. REST acts together with the co-repressor CoREST to silence genes in non-neuronal cells (Ballas and Mandel, 2005; Ooi and Wood, 2007). A recent study by Westbrook and colleagues found that REST is polyubiquitinated and degraded in neural stem cells by $\beta \operatorname{TrCP} 1$ to promote neurogenesis (Westbrook et al., 2008).

Moreover, the C. elegans homologue of $\beta$-TrCP, Lin-23 was originally identified to restrain cell proliferation in response to developmental cues (Kipreos et al., 2000). It was later identified as a cytoplasmic protein that is essential for axon growth (Mehta et al., 2004). Null alleles of Lin-23 gene and a point mutation in the Cterminal tail of the protein both show a defect in axon outgrowth. In addition to axon outgrowth-regulating function, Lin-23 regulates the abundance of glutamate 
receptor subunit GLR1 in the ventral nerve cord of C. elegans. Lin-23-mediated regulation of GLR1 abundance results from degradation of the $\beta$-catenin homologue, BAR-1 (Dreier et al., 2005).

Similar to $\beta \operatorname{TrCP}$, neural differentiation is also promoted by another F-box protein FBXW7. FBXW7 is a cell cycle regulator that targets substrates including Notch1, c-jun, c-myc, cyclin E and mTor for degradation (Welcker and Clurman, 2008). In the context of neural stem cell differentiation, FBXW7 has been shown to promote ubiquitination and degradation of Notch1. Notch signaling is triggered by the intercellular interaction of the ligands delta-like (DLL) or jagged and the Notch receptor. This interaction results in the activation of $\gamma$-secretase that acts on the intracellular domain of Notch receptor to generate NICD (notch intracellular domain) protein. NICD then translocates into the nucleus to activate the bHLH (basic helix loop helix) family of transcriptional repressors, which inhibit neuronal differentiation into neurons (Pierfelice et al., 2011). By promoting the degradation of Notch1, FBXW7 drives neuronal differentiation. Genetic loss of FBXW7 in the neural progenitors in the conditional knockout $F B X W^{\gamma / f / f} ; N e s t i n-C r e\left(\mathrm{FBXW}^{\Delta \mathrm{N}}\right)$ mouse results in decreased neurogenesis and impaired differentiation. This block in neural differentiation is alleviated by downregulation of Notch in FBXW7 ${ }^{\Delta \mathrm{N}}$ background (Hoeck et al., 2010). Besides its role in neuronal differentiation, FBXW7 is identified as a crucial regulator of neuronal migration of granule neurons and thus cerebellar development. FBXW7 conditional knockout mice $\left(F B X W^{r}{ }^{\Delta C b}\right)$, with FBXW7 inactivated in the cerebellar anlage, showed a reduced cerebellar size, reduced Purkinje cell number and aberrant progenitor cell migration. In these mice, Notch1 and N-terminally phosphorylated c-Jun levels are also upregulated. Moreover, deletion of c-jun, a substrate of FBXW7, rescued the Purkinje cell number and arborization in the $F B X W^{r}{ }^{\triangle C b}$ background, suggesting an important role for FBXW7 in cerebellar development (Jandke et al., 2011).

The primary knowledge of F-box proteins regulating neuronal differentiation and migration is derived from studying FBXW1/FBXW11 and FBXW7, that form 
the conventional SCF complex. Recent genetic evidence implicates the F-box protein FBXO45 in neuronal migration. FBXO45 is a brain-abundant F-box protein that forms an atypical ubiquitin ligase with the RING domain protein PAM (protein associated with myc) instead of Rbx1/Roc1. FBXO45 knockout animals show neuronal migration defects in the brain and the spinal cord. The migration defects in the $\mathrm{FBXO}_{4} 5^{-/-}$animals were established by BrdU-pulse labeling, which showed an impaired migration of the cortical neurons (Saiga et al., 2009). Further analysis of the $\mathrm{FBXO}_{4} 5^{-/-}$mice revealed the requirement of FBXO45 in formation of axon tracts and neuromuscular junctions. Impaired formation of neuromuscular junction supports the requirement of FBXO45 in regulating synaptic function. In this context, Tada and colleagues have identified that FBXO45 triggers the degradation of Munc13-1, a synaptic vesicle-priming factor, to regulate synaptic activity (Tada et al., 2010). This is further supported by a study in C. elegans. Fsn-1, the homologue of FBXO45, is located at the pre-synaptic site where it targets receptor tyrosine kinase ALK (anaplastic lymphoma kinase) and stabilizes synapse formation (Liao et al., 2004). Another F-box protein, FBXL20 (SCRAPPER), localized at the presynaptic membrane, induces degradation of RIM1 (Rab3-interacting molecule), a vesicle priming protein (Yao et al., 2007). In scrapper-knockout neurons, RIM1 has a longer half-life and thus an altered synaptic activity is observed with increased frequency of excitatory postsynaptic currents.

Adding to this small family of F-box proteins regulating neuronal morphology, FBXW8 has been the only identified F-box protein regulating dendrite growth in neurons. FBXW8 is localized at the golgi complex and associates with Cul7 where it targets the golgi protein Grasp65 for degradation (Litterman et al., 2011). Thus, only a selected number of F-box proteins have been studied in the context of neuronal development and an even lesser number have been associated with neurological disorders, which are discussed in the next section. 
Table 1.2: F-box proteins in neuronal development

\begin{tabular}{|c|c|c|c|c|}
\hline $\begin{array}{l}\text { F-box pro- } \\
\text { tein }\end{array}$ & $\begin{array}{l}\text { Subcellular } \\
\text { localization }\end{array}$ & $\begin{array}{l}\text { Neuronal } \\
\text { substrate }\end{array}$ & Function & Reference \\
\hline $\begin{array}{l}\text { FBXW1/ } \\
\text { FBXW11 }\end{array}$ & Cytoplasm & $\beta$-catenin & $\begin{array}{l}\text { Progenitor } \\
\text { proliferation }\end{array}$ & (Mao et al., 2009) \\
\hline $\begin{array}{l}(\beta \operatorname{TrCP} 1 / \\
\beta \operatorname{TrCP} 2)\end{array}$ & Cytoplasm & REST & $\begin{array}{l}\text { Neural } \\
\text { differentiation }\end{array}$ & $\begin{array}{l}\text { (Westbrook et al., } \\
2008 \text { ) }\end{array}$ \\
\hline \multirow[t]{2}{*}{ Lin-23 } & Cytoplasm & $?$ & Axon growth & $\begin{array}{l}\text { (Mehta et al., } \\
2004)\end{array}$ \\
\hline & Synapse & Bar-1 & Synaptic activity & $\begin{array}{l}\text { (Dreier et al., } \\
2005)\end{array}$ \\
\hline \multirow[t]{2}{*}{ FBXW7 } & Cytoplasm & Notch & $\begin{array}{l}\text { Neural } \\
\text { differentiation }\end{array}$ & $\begin{array}{l}\text { (Hoeck et al., } \\
2010)\end{array}$ \\
\hline & Cytoplasm & c-jun & $\begin{array}{l}\text { Neuronal } \\
\text { migration }\end{array}$ & $\begin{array}{l}\text { (Jandke et al., } \\
2011 \text { ) }\end{array}$ \\
\hline FBXW8 & Golgi complex & Grasp65 & Dendrite growth & $\begin{array}{l}\text { (Litterman et al., } \\
\text { 2011) }\end{array}$ \\
\hline $\begin{array}{l}\text { FBXL20 } \\
\text { (SCRAP- } \\
\text { PER) }\end{array}$ & Synapse & RIM & Synaptogenesis & (Yao et al., 2007) \\
\hline \multirow[t]{2}{*}{ FBXO45 } & $?$ & $?$ & $\begin{array}{l}\text { Neuronal } \\
\text { migration }\end{array}$ & (Saiga et al., 2009) \\
\hline & Synapse & Munc13-1 & Synaptogenesis & (Tada et al., 2010) \\
\hline Fsn-1 & Synapse & $?$ & Synaptogenesis & (Liao et al., 2004) \\
\hline
\end{tabular}

\subsubsection{SCF complex in neurodegenerative disorders}

Since ubiquitin ligases play a crucial role in neuronal development, a dysregulation of the UPS in the nervous system is often associated with neurological disorders (Mabb et al., 2011). Recently, the SCF complex has been implicated in 
Alzheimer's disease (AD), Parkinson's disease and schizophrenia (summarized in Table 1.3). Alzheimer's disease is characterized by progressive memory loss and severe brain atrophy as a result of deposition of $\beta$-amyloid $(\mathrm{A} \beta)$ plaques (Huang and Mucke, 2012). A $\beta$ is derived from sequential cleavage of amyloid precursor protein (APP) by BACE1 and $\gamma$-secretase. Inhibition of the proteasome system results in accumulation of BACE1 (Qing et al., 2004). BACE1 has been identified as a substrate of FBX2-SCF (Gong et al., 2010). Overexpression of FBX2 in primary neurons derived from transgenic mice ( $\mathrm{Tg} 2576$ ) showing AD phenotype, promoted BACE1 degradation and reduced $\beta$-amyloid production. Another F-box protein FBXO7, a member of the PARK family of proteins, has been associated with earlyonset parkinsonism. Mutations in the $\mathrm{FBXO}^{7}$ gene in three families are associated with an early-onset parkinsonian phenotype including rigidity, tremor and dystonia (Di Fonzo et al., 2009; Paisán-Ruiz et al., 2010).

Another prominent neurological disorder, schizophrenia, is characterized by hallucinations, delusions and disorganized speech resulting in social or occupational dysfunction (Ouzir et al., 2012). Recently, two F-box proteins were associated with schizophrenia. While Chen and colleagues identified an association of FBXL21 with schizophrenia in an Irish family (Chen et al., 2008), Narayan and colleagues report a decrease in expression of F-box protein FBXO31 in schizophrenic patients with short-term illness (Narayan et al., 2008). Besides its association with schizophrenia, FBXO31 has also been linked to microcephaly and intellectual impairment (Butler et al., 2012). A $265 \mathrm{~kb}$ contiguous gene deletion was identified in chromosome 16q24.3 in a patient with clinical symptoms including microcephaly, intellectual impairment and distichiasis. The deletion includes C16ORF95, FBXO31, MAP1LC3B and $Z C C H C 14$. Whereas $Z C C H C 14$ and C16ORF95 encode uncharacterized proteins, MAP1LC3B is homologous to rat Map1lc3 gene that encodes microtubuleassociated proteins 1A/1B light chain 3B. Conditional inactivation of Map1lc3 gene impairs autophagy resulting in neurodegeneration in mice (Hara et al., 2006; Komatsu et al., 2006). FBXO31 encodes F-box protein FBXO31 that forms an SCF 
complex (Kumar et al., 2005). Although FBXO31 expression is enriched in the brain (Kumar et al., 2005), its function in the nervous system has not been described.

Table 1.3: F-box proteins in neurodegenerative diseases

\begin{tabular}{lllll}
\hline $\begin{array}{lll}\text { F-box } \\
\text { protein }\end{array}$ & $\begin{array}{l}\text { Subcellular } \\
\text { localization }\end{array}$ & $\begin{array}{l}\text { Neuronal } \\
\text { substrate }\end{array}$ & $\begin{array}{l}\text { Neurodegene- } \\
\text { rative disease }\end{array}$ & Reference \\
\hline \multirow{2}{*}{ FBXO2 } & $?$ & BACE1 & $\begin{array}{l}\text { Alzheimer's } \\
\text { disease }\end{array}$ & (Gong et al., 2010) \\
FBXL2 & $?$ & Alzheimer's & (Watanabe et al., \\
& & & disease & $2012)$ \\
FBXO7 & $?$ & $?$ & Parkinson's & (Di Fonzo et al., \\
& & & disease & 2009; Paisán-Ruiz \\
FBXL21 & $?$ & $?$ & Schizophrenia & (Ouzir et al., 2012) \\
& & $?$ & Schizophrenia & (Narayan et al., \\
FBXO31 & $?$ & & & $2008)$ \\
\hline
\end{tabular}

\subsection{Aim of the study}

F-box proteins were initially identified as regulators of cell cycle. Interestingly, these cell cycle proteins are also present in the post-mitotic cells such as neurons, suggesting a function beyond cell cycle. Given that 69 F-box proteins have been identified in mammals, only a few have been investigated in context of the developing brain. FBXO31 was identified as a cell cycle regulator, where it induces the degradation of cyclin D1 and mediates G1 to S phase transition (Kumar et al., 2005; Santra et al., 2009). Besides its role as a cell cycle regulator, FBXO31 has been proposed to act as a breast tumor and hepatocellular carcinoma suppressor (Kumar et al., 2005; Huang et al., 2010). While FBXO31 is enriched in the brain (Kumar et al., 2005), its functions in the nervous system remains elusive. 
The aim of the current study was to investigate the role of FBXO31 in neuronal development. The first task was to study the localization of this protein to provide an insight into its probable function in neurons. Next, gain-of-function and loss-of-function approaches were used to evaluate the role of FBXO31 in neuronal morphogenesis. To gain mechanistic insight into the phenotypes observed, novel interaction partners and substrates of FBXO31 were identified and further investigated for their role in neuronal morphogenesis. Taken together, this study is the first to systematically investigate the role of FBXO31-SCF complex in neurons. 
Thomas Huxley

\section{Materials and Methods}

\subsection{Materials}

\subsubsection{Antibodies}

Primary antibodies used in this study are listed in Table 2.1. The antibodies were obtained from DakoCytomation (Carpinteria, CA, USA), Invitrogen (Darmstadt, Germany), Millipore (Billerica, MA, USA), NeuroMab (Davis, CA, USA), Novus Biologicals (Cambridge, UK), Santa Cruz (Santa Cruz, CA, USA), and Sigma-Aldrich (Munich, Germany). Fluorophore-coupled (Cy2 or $\mathrm{Cy} 3$ ) and peroxidase-conjugated antibodies (anti-mouse $\operatorname{Ig} G$ and anti-rabbit $\operatorname{Ig} G$ ) were purchased from Dianova (Hamburg, Germany).

\subsubsection{Chemicals, Enzymes and Kits}

\section{Chemicals}

The chemicals used in the study were primarily obtained from either SigmaAldrich (Munich, Germany), Roth (Karlsruhe, Germany), Merck (Darmstadt, Germany), Applichem (Darmstadt, Germany), GE Healthcare (US), Worthington (UK) or Th. Geyer (Germany) unless otherwise stated. All the chemicals were either of analytical purity or cell culture grade. 
Table 2.1: Antibodies used in this study: IF (Immunofluorescence), WB (Western blot), $r$ : rabbit (affinity purified or serum), $m$ : mouse (monoclonal).

\begin{tabular}{|c|c|c|}
\hline Target & Application & Reference \\
\hline$r$-anti-FBXO31 & IF $(1: 400)$ & Novus Biologicals \\
\hline$m$-anti- $\gamma$-tubulin & $\operatorname{IF}(1: 2500)$ & Sigma-Aldrich \\
\hline$m$-anti-AnkyrinG (clone N 106/36) & IF $(1: 50)$ & NeuroMab \\
\hline$r$-anti-GFP & IF $(1: 1000)$, WB $(1: 5000)$ & Invitrogen \\
\hline$m$-anti-MAP2 & IF $(1: 1000)$ & Santa Cruz \\
\hline$m$-anti- $\beta$ IIItubulin & IF $(1: 2000)$ & Santa Cruz \\
\hline$m$-anti- $\beta$ galactosidase & IF $(1: 100)$ & Santa Cruz \\
\hline$m$-anti-GFP & IF $(1: 100)$, WB $(1: 1000)$ & Santa Cruz \\
\hline$m$-anti-myc & IF $(1: 200)$, WB $(1: 1000)$ & Santa Cruz \\
\hline$m$-anti-Flag & IF $(1: 500)$, WB $(1: 1000)$ & Sigma-Aldrich \\
\hline$m$-anti-HA & WB $(1: 1000)$ & Santa Cruz \\
\hline$r$-anti-ubiquitin & WB $(1: 1000)$ & DakoCytomation \\
\hline$m$-anti-ubiquitin & WB $(1: 1000)$ & Santa Cruz \\
\hline$m$-anti-ubiquitin Lys48-specific & WB $(1: 1000)$ & Millipore \\
\hline$m$-anti-ubiquitin Lys63-specific & WB $(1: 1000)$ & Millipore \\
\hline
\end{tabular}

Lactacystin, Poly-L-ornithine hydrobromide, $N$-Ethylmaleimide (NEM) and Insulin were acquired from Sigma-Aldrich, trypsin from Worthington, albumin fraction $\mathrm{V}$ from Applichem and ECL western blotting substrates from ThermoFischer Scientific. ProteinA-Sepharose beads and Ni-NTA Sepharose beads were purchased from GE Healthcare and Qiagen, respectively.

Cell culture media and supplements like Dulbecco's modified eagle's medium (DMEM), Hank's Balanced Salt Solution (HBSS), Basal Medium Eagle (BME), Neurobasal medium, GlutaMAX ${ }^{\mathrm{TM}}$ and Pen-Strep-Glutamine (PSG), B27 supplement and $0.5 \%$ Trypsin-EDTA were purchased from GIBCO (Invitrogen, Darm- 
stadt, Germany). Poly-L-Lysine (PLL) and goat serum were obtained from SigmaAldrich. Calf serum was purchased from Hyclone (Germany), Fetal bovine serum from Biochrom (Germany) and horse serum from PAA (Cölbe, Germany), respectively.

Consumables were purchased from Falcon (Becton Dickinson Labware Europe, Le Pont De Claix, France) and Eppendorf (Eppendorf AG, Hamburg, Germany). Culture dishes $(6 \mathrm{~cm}$ and $10 \mathrm{~cm}$ ) and plates (6-well, 12-well and 24-well) were obtained from Greiner Bio-One (Greiner Bio-One GmbH, Frickenhausen, Germany).

\section{Buffers and Solution}

The various buffers and solutions used in the study are listed in Table 2.2.

Table 2.2: Buffers and solutions used in the study

\begin{tabular}{|c|c|}
\hline Buffers and solutions & Ingrediants \\
\hline $\begin{array}{l}\text { Phosphate buffer saline } \\
\text { (PBS) }\end{array}$ & $\begin{array}{l}137 \mathrm{mM} \mathrm{NaCl}, 10 \mathrm{mM} \mathrm{KCl}, 20 \mathrm{mM} \mathrm{Na} 2 \mathrm{HPO}_{4}, 20 \mathrm{mM} \\
\mathrm{KH}_{2} \mathrm{PO}_{4} \mathrm{pH} 7.4\end{array}$ \\
\hline $\begin{array}{l}\text { Triton }^{\text {TM }} \text { X-100 Lysis } \\
\text { Buffer }\end{array}$ & $\begin{array}{l}150 \mathrm{mM} \mathrm{NaCl}, 50 \mathrm{mM} \text { Tris- } \mathrm{HCl} \mathrm{pH} 7.5,1 \mathrm{mM} \text { EDTA, } \\
1 \% \text { Triton }^{\mathrm{TM}} \mathrm{X}-100\end{array}$ \\
\hline Co-IP buffer & $\begin{array}{l}150 \mathrm{mM} \mathrm{NaCl}, 20 \mathrm{mM} \text { Tris-HCl } \mathrm{pH} 7.4,1 \mathrm{mM} \text { EDTA, } \\
1 \% \text { Nonidet P-40, } 10 \% \text { glycerol }\end{array}$ \\
\hline RIPA buffer & $\begin{array}{l}150 \mathrm{mM} \mathrm{NaCl}, 50 \mathrm{mM} \text { Tris- } \mathrm{HCl} \mathrm{pH} 7.5,1 \% \text { sodium de- } \\
\text { oxycholate, } 1 \% \text { Nonidet P-40, } 0.1 \% \mathrm{SDS}\end{array}$ \\
\hline 2x YT media & $10 \mathrm{~g} / \mathrm{L}$ Yeast, $16 \mathrm{~g} / \mathrm{L}$ Tryptone, $5 \mathrm{~g} / \mathrm{L} \mathrm{NaCl}$ \\
\hline BL21-Lysis buffer & $\begin{array}{l}10 \mathrm{mM} \text { Tris-HCl pH 7.9, } 10 \% \text { glycerol, } 0.5 \mathrm{M} \mathrm{NaCl}, 0.1 \% \\
\text { Nonidet P-40, } 5 \mathrm{mM} \beta \text {-mercaptoethanol, } 1 \mathrm{mM} \mathrm{PMSF}\end{array}$ \\
\hline BC 100 buffer & $\begin{array}{l}20 \mathrm{mM} \text { Tris- } \mathrm{HCl} \mathrm{pH} 7.9,20 \% \text { glycerol, } 100 \mathrm{mM} \mathrm{KCl}, 5 \\
\mathrm{mM} \beta \text {-mercaptoethanol, } 0.5 \mathrm{mM} \text { PMSF }\end{array}$ \\
\hline Running buffer & $25 \mathrm{mM}$ Tris base, $190 \mathrm{mM}$ glycine, $0.1 \%$ SDS \\
\hline Transfer buffer & $20 \mathrm{mM}$ Tris base, $153 \mathrm{mM}$ glycine, $20 \%$ methanol \\
\hline Upper buffer & 0.5 M Tris-HCl pH 6.8, 0.4\% SDS \\
\hline
\end{tabular}




\begin{tabular}{|c|c|}
\hline Lower buffer & 1.5 M Tris- $\mathrm{HCl} \mathrm{pH} 8.8,0.4 \%$ SDS \\
\hline 5x SDS-sample buffer & $\begin{array}{l}12.5 \mathrm{~mL} \text { upper buffer, } 10 \mathrm{~mL} \text { glycerol, } 2 \mathrm{~g} \text { SDS, } 1 \mathrm{~mL} \\
\beta \text {-mercaptoethanol, bromophenol blue in } 25 \mathrm{~mL} \mathrm{H}_{2} \mathrm{O}\end{array}$ \\
\hline Annealing buffer & $\begin{array}{l}100 \mathrm{mM} \text { potassium acetate, } 30 \mathrm{mM} \text { HEPES-KOH pH 7.4, } \\
2 \mathrm{mM} \text { magnesium acetate }\end{array}$ \\
\hline 2x HBSS buffer & $\begin{array}{l}4 \mathrm{~g} \mathrm{NaCl}, 117.5 \mathrm{mg} \mathrm{KCl}, 95 \mathrm{mg} \mathrm{Na} 2 \mathrm{HPO}_{4} .7 \mathrm{H}_{2} \mathrm{O}, 675 \mathrm{mg} \\
\text { Glucose, } 2.5 \mathrm{~g} \text { HEPES-free acid in } 250 \mathrm{~mL} \text { water }\end{array}$ \\
\hline $2 \mathrm{x}$ TAE & $80 \mathrm{mM}$ Tris-acetate, $2 \mathrm{mM}$ EDTA $\mathrm{pH} 8.5$ \\
\hline 4\% Paraformaldehyde & $4 \mathrm{~g}$ PFA, $4 \mathrm{~g}$ sucrose in $100 \mathrm{~mL} \mathrm{PBS}$ \\
\hline $\begin{array}{l}\text { Mowiol-mounting } \\
\text { medium }\end{array}$ & $\begin{array}{l}6 \mathrm{~g} \text { glycerol AR, } 2.4 \mathrm{~g} \text { Mowiol 4-88 (Calbiochem), } 6 \mathrm{~mL} \\
\mathrm{H}_{2} \mathrm{O}, 12 \mathrm{~mL} 0.2 \mathrm{M} \text { Tris- } \mathrm{HCl} \mathrm{pH} 8.5\end{array}$ \\
\hline HHGN & $\begin{array}{l}\text { 1x HBSS, } 2.5 \mathrm{mM} \text { HEPES pH 7.5, } 35 \mathrm{mM} \text { glucose, } 4 \mathrm{mM} \\
\mathrm{NaHCO}_{3}\end{array}$ \\
\hline BME + Insulin & $35 \mathrm{mM}$ glucose, $1 \mathrm{X}$ PSG, $10 \mu \mathrm{g} / \mathrm{mL}$ insulin in BME \\
\hline
\end{tabular}

\section{Enzymes}

The enzymes used in the study are listed in Table 2.3 along with their application. Enzymes were purchased from Fermantas (St. Leon-Rot, Germany), New England Biolabs (NEB GmbH, Frankfurt, Germany), Promega (Mannheim, Germany) and Roche (Basel, Switzerland).

Table 2.3: Enzymes used in this study

\begin{tabular}{lll}
\hline Enzyme & Application & Reference \\
\hline Restriction enzymes & DNA digest & NEB \\
T4 DNA ligase & ligation of DNA fragments & Fermentas \\
Pfu DNA polymerase & polymerase chain reaction & Fermentas \\
Taq DNA polymerase & polymerase chain reaction & Promega \\
Lambda protein phosphatase & $5^{\prime}$ phosphate removal & NEB \\
T4 polynucleotide kinase & $5^{\prime}$ phosphorylation & NEB \\
\hline
\end{tabular}


Kits

The commercially available kits used in this study are listed in Table 2.4. The kits were used according to the manufacturer's guidelines.

Table 2.4: Commercial kits used in this study

\begin{tabular}{lll}
\hline Kit & Application & Reference \\
\hline NucleoSpin Plasmid kit & DNA isolation, small scale & Macherey-Nagel \\
NucleoBond ${ }^{\mathbb{R}}$ Xtra EF & DNA isolation, medium scale & Macherey-Nagel \\
NucleoSpin Extract II kit & DNA extraction from agarose gels & Macherey-Nagel \\
Nucleospin ${ }^{\circledR}$ Gel & DNA extraction from gel & Macherey-Nagel \\
and PCR Clean-Up & and PCR clean up & \\
Pierce ${ }^{\circledR}$ ECL substrate & western blotting & ThermoFischer \\
Bio-Rad Protein Assay & protein quantification & Bio-Rad \\
\hline
\end{tabular}

\subsubsection{Mammalian cell lines and bacterial strains}

The mammalian cell lines and the bacterial strains used in the study are indicated in Table 2.5 .

Table 2.5: Cell lines and bacterial strains employed in the study

\begin{tabular}{|c|c|}
\hline Cells & Description \\
\hline HEK $293 \mathrm{~T}$ & human embryonic kidney $293 \mathrm{~T}$ \\
\hline Neuro-2a & mouse neuroblastoma cell line \\
\hline E. coli $\mathrm{DH} 5 \alpha$ & $\begin{array}{l}\mathrm{F}^{-} \Phi 80 \text { lacZ } \theta(\text { lacZYA--argF }) \mathrm{U} 169 \text { rec } \mathrm{A} 1 \text { end } \mathrm{A} 1 \text { hsdR17 } \\
\left(\mathrm{r}_{\mathrm{k}}{ }^{-}, \mathrm{m}_{\mathrm{k}}{ }^{+} \text {phoA supE44 thi-1 gyr } 96 \text { relA1 } \lambda^{-}\right.\end{array}$ \\
\hline E. coli $\mathrm{BL} 21$ & 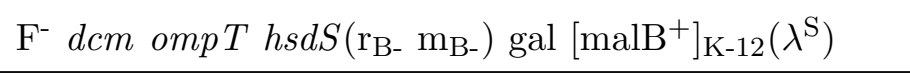 \\
\hline
\end{tabular}




\subsubsection{Softwares used in the study}

The softwares used in the study for image acquisition, image processing, data quantification, plotting of data and statistical analysis are listed in Table 2.6.

Table 2.6: List of softwares used in the study

\begin{tabular}{lll}
\hline Software & Application & Source/Manufacturer \\
\hline NIS-Element & $\begin{array}{l}\text { Image-acquisition } \\
\text { and analysis }\end{array}$ & Nikon \\
ImageJ & Image analysis & http://rsbweb.nih.gov/ij/ \\
Imaris 7.4.2 & Image analysis & Bitplane \\
GraphPad Prism 5.0c & Statistical analysis & GraphPad Software, Inc. \\
WinGlow & Luciferase assay & Berthold Technologies \\
\hline
\end{tabular}

\section{$2.2 \quad$ Methods}

\subsubsection{Molecular biology and biochemical methods}

\section{Molecular Cloning}

Molecular cloning was performed using standard methods of restriction digestion, ligation and transformation into competent E.coli DH5 $\alpha$. The transformed bacteria were plated and grown on 2xYT-agar plate with desired antibiotic. The clones were screened via restriction digestion of plasmid obtained by 'mini-prep' followed by sequencing. The plasmid DNA was obtained using 'midi-prep' kits for positive clone with correct sequences. DNA primers and sequencing results were provided by AGCT lab (Central facility at the Max Planck Institute of Experimental Medicine, Göttingen, Germany). 


\section{Protein determination}

Protein concentration was determined using the standard Bradford method. Protein standards were prepared in the range of 5-15 $\mu \mathrm{g}$ using bovine serum albumin (BSA, Fermentas). The standards were further diluted in solution containing 200 $\mu \mathrm{L}$ bradford solution (Bio-Rad) and $800 \mu \mathrm{L} \mathrm{H}_{2} \mathrm{O}$. The absorbance of the samples was measured at $595 \mathrm{~nm}$ wavelength using a spectrophotometer (Amersham Biosciences). From the standard curve (linear trace), unknown protein concentrations for the test samples were determined.

\section{SDS-PAGE and Western blotting}

Samples were separated on 8-10\% denaturing Tris-SDS polyacrylamide gel electrophoresis system. The resolving gel contained 8-10\% acrylamide/bis-acrylamide (37.5:1, Th. Geyer), 375 mM Tris-HCl (pH 8.5), 0.1\% SDS, 0.05\% ammonium per sulphate (APS) and 0.005\% TEMED (N', N', N', N'-Tetramethyethylenediamine); and the stacking gel had 4\% acrylamide/ bis-acrylamide (37.5:1, Th. Geyer), 125 $\mathrm{mM}$ Tris- $\mathrm{HCl}$ (pH 6.8), 0.1\% SDS, 0.05\% ammonium per sulphate (APS) and $0.005 \%$ TEMED. The samples were boiled in SDS-sample buffer for $5 \mathrm{~min}$ at $95^{\circ} \mathrm{C}$ before loading. For each gel, $3 \mu \mathrm{L}$ of pre-stained protein ladder (Fermentas) was loaded into one of the lanes. Electrophoresis of protein samples was carried out in running buffer at constant current for $1 \mathrm{hr}$.

Proteins were transferred onto Protran BA85 membranes (VWR, Germany) via a semi-dry procedure in the transfer buffer. For the transfer, $40 \mathrm{~mA}$ current was applied for $1 \mathrm{hr}$ in a Bio-rad PowerPac 1000 blotting system. The membranes were then blocked with $4 \%$ non-fat dried milk powder (Granovita GmbH, Germany) dissolved in PBST (0.1\% Tween-20 in PBS), for 30 min followed by overnight incubation in primary antibodies (diluted appropriately in PBST). Following primary antibody incubation, blots were washed three times, 10 min each, in PBST and then incubated with secondary antibodies (peroxidase conjugated, diluted 1:5000 in $4 \%$ milk in PBST) for $1 \mathrm{hr}$ at RT. Afterwards, blots were washed three times with 
PBST (10 min each). Finally, protein bands were detected on X-ray films (High performance Hyperfilms, Thermo Scientific, Pierce) using enhanced chemiluminescence (ECL, Thermo Scientific, Pierce) as a substrate for peroxidase on kodak imaging station.

\subsubsection{RNA interference}

\section{Generation of vector-based RNAi constructs}

The RNAi constructs targeting three different regions of FBXO31 are listed in Table 2.7. The constructs were generated by sub-cloning an inverted repeat into pBlueScript/U6 at the ApaI site. Each of 40-nucleotide-long inverted repeats, separated by 9-nucleotide loop containing HindIII restriction site, was inserted downstream of U6 promoter. EcoRI restriction site was inserted downstream of antisense strand to provide blunt end for ligation with the vector while the ApaI restriction site provided the sticky end. The sense strand of the hairpin was homologous to a 20-nucleotide region in the target FBXO31 mRNA (coding sequences 669-689; 832852 and 979-999 from the start codon of the human sequence and targets rat, mouse and human FBXO31 genes). U6/Par6c RNAi construct was generated in a similar manner against a previously described target sequence (Zhang and Macara, 2008) listed in Table 2.7. U6/Cdh1 RNAi plasmid was kindly provided by Dr. Judith Stegmüller with a previously described target sequence (Konishi et al., 2004).

Table 2.7: short-hairpin RNA used in this study

\begin{tabular}{lll}
\hline RNAi & Targeting region & Targeting sequence $\left(\mathbf{5}^{\prime}-\mathbf{3}^{\prime}\right)$ \\
\hline FBXO31 RNAi\#1 & $669-689 b p$ & AGGATGAGTTCTCCACCAAGT \\
FBXO31 RNAi\#2 & $832-852 b p$ & CAGTCAGTACGACAACTGCCT \\
FBXO31 RNAi\#3 & $979-999 b p$ & CAGGGGCACCAAGATCACGGG \\
Par6c RNAi & $638-656 b p$ & ATGAGATCCTCGAGGTCAA \\
Cdh1 RNAi & $300-320 b p$ & GGGTGCCGGCATCGAGAAGGT \\
\hline
\end{tabular}




\section{Annealing of primers}

Annealing of sense and antisense primer pairs ( $2 \mu \mathrm{L}$ each) was carried out by diluting the primers $(2 \mu \mathrm{L}$ each of $50 \mathrm{pmol} / \mu \mathrm{L})$ in $46 \mu \mathrm{L}$ of annealing buffer. The annealing of primer pairs was carried out as shown in Table 2.8.

Table 2.8: Annealing of primers

\begin{tabular}{ll}
\hline Temperature & Time \\
\hline $95^{\circ} \mathrm{C}$ & $4 \mathrm{~min}$ \\
$4^{\circ} \mathrm{C}$ & $10 \mathrm{~min}$ \\
Slow cool $0.1^{\circ} \mathrm{C} / \mathrm{sec}$ & \\
\hline
\end{tabular}

\section{Phosphorylation of oligos}

$20 \mu \mathrm{L}$ of annealed oligos was mixed with $5 \mu \mathrm{L}$ of 10x PNK buffer, 10U of polynucleotide kinase (PNK) and ATP to a final concentration of $1 \mathrm{mM}$ in a $50 \mu \mathrm{L}$ kinase reaction incubated at $37^{\circ} \mathrm{C}$ for $30 \mathrm{~min}$. $10 \mu \mathrm{L}$ of phosphorylated oligo was used for ligation.

\section{Preparation of U6/pBS vector and ligation}

$5 \mu \mathrm{g}$ of $\mathrm{pBS} / \mathrm{U} 6$ vector was digested with ApaI for $1 \mathrm{hr}$ at $37^{\circ} \mathrm{C}$ and the linearized vector was purified using the gel extraction columns (NM kit). The linearized plasmid was then incubated with T4 DNA polymerase at $12^{\circ} \mathrm{C}$ for $15 \mathrm{~min}$ to generate blunt ends. This was followed by digestion of the vector with EcoRI at $37^{\circ} \mathrm{C}$ for $1 \mathrm{hr}$ and addition of calf intestinal phosphatase (CIP) for $1 \mathrm{hr}$ to remove $5^{\prime}$ phosphate groups. The vector was purified using column and eluted in $50 \mu \mathrm{L}$ sterile water. Subsequently, a $20 \mu \mathrm{L}$ ligation reaction was set up with $10 \mu \mathrm{L}$ phosphorylated oligos, $1 \mu \mathrm{L}$ digested vector, $2 \mu \mathrm{L}$ 10x Ligase buffer and $1 \mu \mathrm{L}$ T4 DNA ligase. The reaction was incubated at $16^{\circ} \mathrm{C}$ overnight.

\section{Screening for clones}

The E.coli DH5 $\alpha$ cells were transformed with the ligation mix using the standard transformation protocol (heat shock). The cells were plated on 2xYT plate 
with $1.5 \%$ agar and ampicillin and the plates were incubated at $37^{\circ} \mathrm{C}$ overnight. The colonies were cultured in 2xYT media (supplemented with ampicillin) overnight and plasmid DNA was isolated from an individual colony. The plasmid DNA was digested with HpaI enzyme to screen for positive clones. Upon digestion with HpaI, clones with desired RNAi insert gave a linear band of approximately $3 k b p$. The plasmid DNA from positive clones was submitted for sequencing in the AGCT lab.

\subsubsection{Site-directed mutagenesis}

Site-directed mutagenesis was performed to generate rescue constructs resistant to degradation by RNAi constructs used in the study. Primers with 4-6 silent mutations were designed according to the specifications provided in the Quik Change site-directed mutagenesis manual (Stratagene) and the PCR reaction (10 ng plamid DNA, 10 pmol forward primer, 10 pmol reverse primer, 10x Pfu DNA polymerase buffer, $1 \mu \mathrm{L}$ Pfu DNA polymerase, $25 \mathrm{mM}$ dNTP in $50 \mu \mathrm{L}$ reaction) was set as shown in table Table 2.9.

The samples were then incubated on ice for 2 min followed by addition of DpnI to the reaction mix for $1 \mathrm{hr}$ at $37^{\circ} \mathrm{C} .1-2 \mu \mathrm{L}$ of reaction mix was used to transform E.coli DH5 $\alpha$ competent cells, plated on 2xYT-agar plate and incubated overnight at $37^{\circ} \mathrm{C}$. The clones were sequenced to confirm the mutations in the vector.

Table 2.9: Site-directed mutagenesis PCR

\begin{tabular}{ll}
\hline Temperature & Time \\
\hline $95^{\circ} \mathrm{C}$ & $3 \mathrm{~min}$ \\
$95^{\circ} \mathrm{C}$ & $30 \mathrm{sec}$ \\
$55^{\circ} \mathrm{C}$ & $30 \mathrm{sec}$ \\
$72^{\circ} \mathrm{C}$ & $500 \mathrm{bp} / \mathrm{min}-18$ cycles \\
$72^{\circ} \mathrm{C}$ & $5 \mathrm{~min}$ \\
\hline
\end{tabular}




\subsubsection{Quantitative analysis of gene expression}

\section{RNA isolation and cDNA synthesis}

P6, P12 and 4-month-old adult wistar rats were anaesthetized in a $\mathrm{CO}_{2}$ chamber and sacrificed. Different tissue samples including cortex, hippocampus, cerebellum, olfactory bulb, heart, lung, liver, kidney and spleen were isolated, rapidly frozen in liquid $\mathrm{N}_{2}$ and stored at $-80^{\circ} \mathrm{C}$. Total RNA was extracted from approximately 30-40 mg of tissue using Trizol reagent. $3 \mu \mathrm{g}$ of total RNA of each tissue sample was used for cDNA synthesis with the SuperScript double strand cDNA synthesis kit according to instruction manual. Polymerase chain reaction (PCR) was performed for housekeeping gene $\beta$-actin to confirm faithful reverse transcription.

\section{Quantitative PCR}

qPCR was performed on Roche Lightcycler with the primer pairs indicated in Table 2.10. The FBXO31 levels were normalized to $\beta$-actin and represented relative to the cortex values for each age group.

Table 2.10: Primers for quantitative PCR

\begin{tabular}{ll}
\hline Primer & Sequence $\left(\mathbf{5}^{\prime}-\mathbf{3}^{\prime}\right)$ \\
\hline FBXO31 sense & CCACTGTTTTAGAATCCATCTGATGGA \\
FBXO31 anti-sense & ACTTGGTGGAGAACTCGTCCC \\
$\beta$-actin sense & CTTCCTCCCTGGAGAAGAGC \\
$\beta$-actin anti-sense & ATGCCACAGGATTCCATACC \\
\hline
\end{tabular}

\subsubsection{Biochemical assays}

\section{Cell lysis}

HEK 293 T cells (90\% confluent) or cerebellar granule neurons (5 x $10^{6}$ cells per well) were grown in 6-well plates for the desired time periods. After aspirating off the medium, cells were washed once with PBS and scraped in 100-300 $\mu \mathrm{L}$ of 
lysis buffer (Table 2.2) supplemented with fresh protease inhibitors (1 mM DTT, 1 $\mu \mathrm{g} / \mathrm{mL}$ pepstatin, $3 \mu \mathrm{g} / \mathrm{mL}$ aprotinin and $1 \mu \mathrm{g} / \mathrm{mL}$ leupeptin) on ice and further incubated for $30 \mathrm{~min}$ on ice. Following incubation in the lysis buffer, the samples were centrifuged (pre-cooled bench top centrifuge, Eppendorf, Germany) at 13,000 rpm for $10 \mathrm{~min}$. The supernatant was collected in a fresh tube and protein concentration was determined using bradford assay (2.2.1).

\section{Co-immunoprecipitation}

Transfected HEK 293T cells were washed with PBS and lysed in coimmunoprecipitation (co-IP) buffer (Table 2.2) supplemented with fresh protease inhibitors (1 mM DTT, $1 \mu \mathrm{g} / \mathrm{mL}$ pepstatin, $3 \mu \mathrm{g} / \mathrm{mL}$ aprotinin and $1 \mu \mathrm{g} / \mathrm{mL}$ leupeptin). The cell lysates were incubated on ice for $30 \mathrm{~min}$ and centrifuged at 13,000 rpm for 10 min at $4^{\circ} \mathrm{C}$. The supernatant was transferred to fresh tube and total protein amount was estimated using Bradford reagent. $1 \mathrm{mg}$ of total protein lysate was incubated with $0.2-0.8 \mu \mathrm{g}$ of antibody for $2 \mathrm{hrs}$ at $4^{\circ} \mathrm{C}$ on rotor. $30 \mu \mathrm{g}$ of total protein lysate was separately boiled with SDS-sample buffer and used as input. $20 \mu \mathrm{L}$ of Protein A Sepharose beads (GE Healthcare), equilibrated with 1:1 co-IP buffer, was added to the cell lysate and incubated for $1 \mathrm{hr}$ at $4^{\circ} \mathrm{C}$ on rotor. The lysate was then centrifuged at 13,000 rpm for $1 \mathrm{~min}$ and the pellet containing the beads was washed three times with lysis buffer. The final wash was done with PBS. The sample was spun down and the PBS was carefully removed. The protein bound to beads was eluted by boiling the samples in SDS-sample buffer at $95^{\circ} \mathrm{C}$ for $5 \mathrm{~min}$. The samples were subjected to analysis by SDS-PAGE and western blotting.

\section{Cell-based ubiquitination assay}

Transfected HEK 293T cells were lysed in co-IP buffer supplemented with fresh protease inhibitors $(1 \mathrm{mM}$ DTT, $1 \mu \mathrm{g} / \mathrm{mL}$ pepstatin, $3 \mu \mathrm{g} / \mathrm{mL}$ aprotinin and $1 \mu \mathrm{g} / \mathrm{mL}$ leupeptin) and $10 \mathrm{mM}$ NEM. The cell lysates were incubated on ice for 30 min, centrifuged and the supernatant was transferred to a fresh tube. Total protein 
concentration was estimated using the Bradford assay and $1 \mathrm{mg}$ of total protein lysate was used for co-immunoprecipitation with the first antibody for 2 hrs at $4^{\circ} \mathrm{C}$. $30 \mu \mathrm{g}$ of lysate from each sample was boiled separately with SDS-sample buffer to serve as input. $20 \mu \mathrm{L}$ of Protein-A sepharose beads, equilibrated in co-IP buffer, was added to the lysates and incubated for $1 \mathrm{hr}$ at $4^{\circ} \mathrm{C}$. The samples were centrifuged at $13000 \mathrm{rpm}$ at $4^{\circ} \mathrm{C}$ for $1 \mathrm{~min}$ and the pellet was washed with lysis buffer and PBS as described earlier. The beads were then boiled with SDS-sample buffer and the samples were analyzed with SDS-PAGE and western blotting by probing with anti-ubiquitin antibody.

\section{S proteasome inhibition}

Cerebellar granule neurons were treated with vehicle (DMSO) or lactacystin (5 $\mu \mathrm{M}) 10 \mathrm{hrs}$ prior to lysis at DIV 3. Transfected cerebellar granule neurons (DIV 2) were treated with vehicle (DMSO) or lactacystin $(5 \mu \mathrm{M}) 10$ hrs prior to lysis at DIV 6. The neurons were lysed in lysis buffer and equal amounts of protein were analyzed by SDS-PAGE and western blotting.

\section{Luciferase assay}

Expression plasmid encoding Renilla-Par6c and Renilla-Cyclin D1 fusion proteins were generated by Dr. Judith Stegmüller by cloning Renilla sequence upstream of Par6c and Cyclin D1, respectively, in pCMVmyc vector. HEK 293T cells were cultured on 24-well plate and transfected (3 wells/condition) with $2 \mu \mathrm{g}$ of control vector or FBXO31 together with $0.1 \mu \mathrm{g}$ Renilla-Par6c and $0.1 \mu \mathrm{g}$ SV40 firefly luciferase (pGL3 promotor) expression plasmid. The cells were lysed 2 days after transfection with $100 \mu \mathrm{L}$ well of passive lysis buffer. The lysates were rocked for 20 min, centrifuged at $13000 \mathrm{rpm}$ for $10 \mathrm{~min}$ and supernatant was transferred to fresh tube. $30 \mu \mathrm{L}$ of lysates per condition were loaded on a 96-well plate in triplicates and the plate was subjected to dual-luciferase assay with firefly and renilla substrates in the luminometer. Sabrina Galinski assisted in obtaining firefly and renilla luciferase 
activity readings using WINGLOW software. The values were exported to Microsoft Excel for further analysis. The ratio of renilla to firefly activity indicated normalized protein expression of the respective constructs.

\section{Purification of FBXO31 antigen}

To generate the FBXO31 antigen, FBXO31 coding sequence (1224 -1521 bp encoding 100 aa at C-terminus) was PCR amplified from wild-type BL/6 mouse cDNA and cloned into pET3a expression vector. E. coli BL21 cells were transformed with pET3a/FBXO31 expression plasmid and grown on 2xYT-agar plate with ampicillin. Single colony was isolated, grown overnight in $50 \mathrm{~mL}$ 2xYT medium (supplemented with $50 \mu \mathrm{g} / \mathrm{mL}$ ampicillin) and appropriate volume was transferred to $1 \mathrm{~L}$ of fresh $2 \mathrm{xYT}$ to obtain an O.D. of 0.1. The culture was further incubated at $37^{\circ} \mathrm{C}$ to an O.D. of 0.6 , induced with $0.5 \mathrm{mM}$ Isopropyl $\beta$-D-1-thiogalactopyranoside (IPTG) and grown for additional 3 hrs. The cells were harvested by centrifugation and the pellet was lyzed in $20 \mathrm{~mL}$ BL21-lysis buffer with freshly added $1 \mathrm{mM}$ PMSF by sonication. After centrifugation for $30 \mathrm{~min}$ at $37,000 \mathrm{rpm}$, the cleared lysate was incubated with $250 \mu \mathrm{L}$ Ni-NTA Sepharose beads (Qiagen) and $1 \mathrm{mM}$ imidazole and incubated overnight at $4^{\circ} \mathrm{C}$ on rotor. The sample was centrifuged at $4000 \mathrm{rpm}$ and beads were incubated with $5 \mathrm{~mL}$ BC100 buffer with $20 \mathrm{mM}$ imidazole for $30 \mathrm{~min}$ at $4^{\circ} \mathrm{C}$ on rotor. The sample was centrifuges again and $2.5 \mathrm{~mL}$ BC100 buffer with $40 \mathrm{mM}$ imidazole was added to the beads. The beads were inbucated on rotor for $30 \mathrm{~min}$ at $4^{\circ} \mathrm{C}$. In the last step, beads were washed in $250 \mu \mathrm{L}$ BC100 with $200 \mathrm{mM}$ imidazole and the protein was eluted. The samples were subjected to analysis by SDS-PAGE as described previously.

\subsubsection{Cell culture}

\section{HEK 293T cell culture}

HEK 293T cells were grown in DMEM medium (4.5 g/L glucose; [-] glutamine; [-] pyruvate, GIBCO) supplemented with 10\% FBS (Biochrom) and 1\% 
GlutaMAX $^{\mathrm{TM}}$ (Invitrogen) in $10 \mathrm{~cm}$ culture dishes. The cells were split when the plate was approximately $90 \%$ confluent. The cells were washed once with PBS after removing the media and $2 \mathrm{~mL}$ of $1 \mathrm{x}$ TE $(10 \mathrm{mM}$ Trypsin $\mathrm{pH} 8.0$ and $1 \mathrm{mM}$ EDTA) was added to the cells. The plate was incubated at $37^{\circ} \mathrm{C}$ for $2 \mathrm{~min}$. The adhering cells were removed by adding $8 \mathrm{~mL}$ of serum-supplemented DMEM and the cells were pipetted up and down several times to obtain single-cell suspension. The cell suspension was centrifuged at $800 \mathrm{rpm}$ for $5 \mathrm{~min}$ and then the pellet was resuspended in $5 \mathrm{~mL}$ of serum-supplemented DMEM. The cells were then plated on either 24-well plate or 6-well plate or $10 \mathrm{~cm}$ dish to obtain desired confluency.

\section{Cerebellar granule neuron culture}

Cerebella were isolated from postnatal day 6 (P6) wistar rat pups in HHGN medium (10x HBSS [-] calcium [-] magnesium, GIBCO; 2.5 mM HEPES pH 7.3; $0.35 \mathrm{M}$ glucose and $0.04 \mathrm{M} \mathrm{NaHCO}_{3}$ ) on ice. Meninges and large blood vessels were removed under the dissection microscope (Nikon) using fine forceps. The cerebella were then transferred to $50 \mathrm{~mL}$ conical tubes and washed three times with HHGN medium. After last wash, $5 \mathrm{~mL}$ of TDn (50 mg Trypsin (Worthington) and $0.5 \mu \mathrm{g}$ DNase in $5 \mathrm{~mL}$ HHGN) was added and incubated in the water bath for $10 \mathrm{~min}$ at $37^{\circ} \mathrm{C}$ followed by $10 \mathrm{~min}$ incubation at room temperature. The cerebella were again washed three times with HHGN media to remove TDn followed by addition of DnB $(0.4 \mu \mathrm{g}$ DNase in $4 \mathrm{~mL}$ BME). The cerebella were triturated to obtain a homogenous suspension and were kept on ice for $5 \mathrm{~min}$. The supernatant was transferred to a fresh $50 \mathrm{~mL}$ conical tube. The pellet was re-triturated with fresh DnB to get single-cell suspension. Both suspensions were mixed and centrifuged at 800 rpm for $5 \mathrm{~min}$. The pellet was resuspended in final volume of $20 \mathrm{~mL}$ Cbc medium (Basal Eagle Medium [-] Glutamine (GIBCO); $10 \%$ calf serum (Hyclone); 1\% PSG (GIBCO) and $25 \mathrm{mM} \mathrm{KCl}$ ). Cells were counted using a hemocytometer. The cells were plated on $12 \mathrm{~mm}$ coverslips pre-coated with polyornithine in 24-well plate at density of $8 \times 10^{5}$ cells/coverslip. For a 6 -well plate, the cells were plated at a density 
of $30 \times 10^{6}$ cells/plate. To prevent the proliferation of non-neuronal cells, the cells were treated with $10 \mathrm{mM}$ anti-mitotic agent cytosine-b-D-arabinofuranoside (AraC) at day in vitro 1 (DIV 1). $35 \mu \mathrm{L}$ of $1 \mathrm{M}$ glucose was added per $\mathrm{mL}$ of conditioned media at DIV 3 to replenish the carbon source in the medium.

\section{Hippocampal neuron culture}

Hippocampal neurons were prepared from hippocampi isolated from E18 wistar rat embryos. The hippocampi were collected in 1x HBSS medium (BME [-] calcium [-] magnesium, GIBCO) and were washed three times with 1x HBSS media. After washes, the hippocampi were incubated in $1.8 \mathrm{~mL} 1 \mathrm{x}$ HBSS with $200 \mu \mathrm{L}$ TE at $37^{\circ} \mathrm{C}$ for $10 \mathrm{~min}$. They were again washed thrice with $1 \mathrm{x}$ HBSS followed by trituration in plating media (DMEM $[+] 4.5 \mathrm{~g} / \mathrm{L}$ glucose $[+]$ glutamine $[+]$ pyruvate (GIBCO); $10 \%$ fetal bovine serum; $1 \%$ PSG and $12.5 \mu \mathrm{M}$ glutamate) with freshly added DNase $(2 \mu \mathrm{g} / \mu \mathrm{L})$. The cells were centrifuged at $800 \mathrm{rpm}$ for $5 \mathrm{~min}$. The pellet was resuspended in $5 \mathrm{~mL}$ of plating media and the cells were counted using a hemocytometer. The cells were plated poly-L-lysine coated $12 \mathrm{~mm}$ coverslips at a density of $1 \times 10^{5}$ cells/coverslip. The plating media was replaced by growth media (Neurobasal medium (GIBCO); 2\% B27 supplement (Invitrogen); 1\% PSG) at DIV 1 to prevent cell death due to excitotoxicity.

\subsubsection{Transfection of primary and secondary cells}

\section{Transfection of HEK 293T cells}

HEK 293 T cells were transfected using the modified calcium phosphate transfection protocol described previously (Konishi et al., 2004). To prepare the DNAcalcium phosphate precipitate for one well of a 6 -well plate, $0.1-2 \mu \mathrm{g}$ of plasmid DNA construct together with 50 ng GFP expression construct (C1-GFP) was diluted in $90 \mu \mathrm{L}$ of sterile water. To this DNA mix, $10 \mu \mathrm{L}$ of $2.5 \mathrm{M} \mathrm{CaCl}_{2}$ was added. The DNA-calcium phosphate solution was buffered by adding $100 \mu \mathrm{L}$ of $2 \mathrm{x}$ HBSS buffer. 
The solution was mixed well to obtain a homogenous DNA precipitate mix that was incubated at RT for $5 \mathrm{~min}$. $200 \mu \mathrm{L}$ of DNA precipitate was added gently to the cells and the plate was slowly rocked to mix the medium. The cells were incubated in the humidified- $\mathrm{CO}_{2}$ incubator at $37^{\circ} \mathrm{C}$ for $2-4$ days and thereafter lysed for biochemical experiments. Transfection efficiency was monitored by checking for GFP fluorescence using the Nikon epifluorescence microscope (Nikon).

\section{Transfection of primary neurons}

Cerebellar granule neurons (CGNs) and hippocampal neurons were transfected using a modified calcium phosphate transfection protocol as described in previous section. CGNs were transfected at DIV 0 (8 hrs after plating) whereas the hippocampal neurons were transfected at DIV 1 for morphological assays. The conditioned media was collected from the primary neurons in a fresh conical tube and incubated at $37^{\circ} \mathrm{C}$ in the water bath. The neurons were washed twice with pre-warmed DMEM (GIBCO) and after second wash $500 \mu \mathrm{L}$ of DMEM was added per well of a 24-well plate. The serum-deprived neurons were incubated in a humidified chamber at $37^{\circ} \mathrm{C}$ for $45 \mathrm{~min}$. During this time, DNA precipitate was prepared. $1-2 \mu \mathrm{g}$ of plasmid DNA construct together with $0.2 \mu \mathrm{g}$ of GFP expression plasmid and $0.3 \mu \mathrm{g}$ of pro-survival factor $\mathrm{Bcl}-\mathrm{x}_{\mathrm{L}}$ was diluted in $18 \mu \mathrm{L}$ of sterile water. $2 \mu \mathrm{L}$ of $2.5 \mathrm{M}$ $\mathrm{CaCl}_{2}$ was added and mixed to the DNA solution which was then buffered with 20 $\mu \mathrm{L}$ of $2 \mathrm{x}$ HBSS. The DNA-calcium phosphate precipitate was incubated for $5 \mathrm{~min}$ at RT and then was added to the serum-deprived cells for $18 \mathrm{~min}$. The cells were then washed twice with DMEM, replaced with conditioned media and incubated in humidified $-\mathrm{CO}_{2}$ chamber.

\subsubsection{Immunocytochemistry}

Primary neurons were cultured on $12 \mathrm{~mm}$ coverslips and transfected as described in section 2.2.7. At specified time-points, they were fixed with $4 \%$ PFA for 10 min and permeabilized with $0.4 \%$ Triton $^{\mathrm{TM}}-\mathrm{X} 100$ in PBS. The neurons were 
blocked with serum-supplemented BME for 30 min and incubated with primary antibody for $1 \mathrm{hr}$. The coverslips were washed with PBS and incubated with Cy2or Cy3-conjugated secondary antibodies for $30 \mathrm{~min}$. The cells were later incubated with the DNA dye Hoechst 33258 (bis-Benzimide) diluted in PBS to stain the nuclei. Epifluorescence microscope (Nikon) was used to detect the fluorescent signal and to capture the images.

For endogenous FBXO31 staining, the cells were washed twice with PBS and then fixed with ice-cold methanol for $5 \mathrm{~min}$ at $-20^{\circ} \mathrm{C}$. The cells were washed again with PBS several times to remove methanol followed by blocking in $10 \%$ goat serum diluted in PBS for 30 min. Rabbit anti-FBXO31 antibody (Novus Biologicals, 1:400) was diluted in PBS with $1 \%$ goat serum and added to cells for $1 \mathrm{hr}$. The cells were washed with PBS and incubated with goat anti-rabbit Cy2 (Dianova), diluted in PBS with $1 \%$ goat serum, for 45 min. The cells were incubated with Hoechst 33258 to stain the nuclei, washed with PBS and mounted using mowiol. For co-localization analysis, the images were captured using laser scanning confocal microscope (Leica).

\subsubsection{Survival assay for cerebellar granule neurons}

CGNs were prepared as described previously and transfected at DIV 2 using modified calcium phosphate transfection protocol. $2 \mu \mathrm{g}$ of $\mathrm{U} 6$ control plasmid or U6/FBXO31 RNAi plasmids together with $0.2 \mu \mathrm{g}$ of $\beta$-galactosidase encoding plasmid was used for transfection. The cells were fixed at DIV 6 using $4 \%$ PFA and stained with mouse monoclonal anti- $\beta$-galactosidase antibody (Santa Cruz). Number of living and dead cells were counted by assessing for integrity of neuronal arbors and morphology of the nucleus in a blinded-manner.

\subsubsection{In vivo electroporation}

Electroporation of P4 wistar rat pups was carried out as described previously (Konishi et al., 2004). For one P4 rat pup, 3-4 $\mu \mathrm{L}$ of plasmid DNA ( $4 \mu \mathrm{g} / \mu \mathrm{L}$ control 
U6 vector or U6/FBXO31 RNAi encoding vector co-expressing GFP together with $1 \mu \mathrm{g} / \mu \mathrm{L} \mathrm{Bcl-x_{ \textrm {L } }}$ ) was used for electroporation. The pups were anaesthetized using isofluorane in a closed chamber and the cerebellum was detected against a bright light source. The plasmid DNA-containing solution diluted in PBS with $0.3 \%$ fast green was injected into the cerebellar cortex using a Hamilton syringe (Hamilton Company) with a 30-gauge needle. Electrical pulses (160-170 V, 50 ms pulses, 950 ms pulse intervals, 5 pulses) were applied using ECM 830 square wave electroporator (Harvard Apparatus). Following electrical stimulation, the pups were allowed to recover under infra red light source and then placed back into the cage with the mother. The pups were sacrificed 5 days after electroporation at P9 and GFPpositive cerebella were screened using upright epifluorescence microscope (Zeiss). GFP-positive cerebella were fixed in 4\% PFA overnight and then transferred to $30 \%$ sucrose solution. After the cerebella had completely sunk into the $30 \%$ sucrose solution, they were embedded in O.C.T compound (TissueTek; Sakura) with $30 \%$ sucrose in 1:1 ratio using embedding moulds. Coronal sections $(40 \mu \mathrm{m})$ of cerebellum were cut on cryostat (Leica). All sections were collected on SuperFrost Ultra Plus slides (Thermo Fisher Scientific) and the GFP-positive sections were processed for immunohistochemistry.

\subsubsection{Immunohistochemistry}

The electroporated cerebella were sectioned coronally $(40 \mu \mathrm{m})$ using the cryostat (Lecia). The sections were mounted on SuperFrost Ultra Plus slides. After air-drying the slides, they were washed with PBS twice and GFP-positive sections were screened using the epifluorescence microscope (Nikon). Sections were blocked in $10 \%$ goat serum in PBS with $0.4 \%$ Triton $^{\mathrm{TM}} \mathrm{X}-100$ for 30 min at RT. The sections were washed with PBS and incubated with anti-mouse GFP antibody (1:100; Santa Cruz) diluted in $1 \%$ goat serum in PBS- $0.4 \%$ Triton $^{\mathrm{TM}} \mathrm{X}-100$ overnight at $4^{\circ} \mathrm{C}$. The slides were then washed three times with PBS for 10 min each. Secondary antibody (goat anti-mouse Cy2; 1:1000) diluted in $1 \%$ goat serum in PBS- $0.4 \%$ Triton $^{\text {TM }}$ X- 
100, was added for $2 \mathrm{hrs}$ at RT. The slides were subsequently washed three times with PBS and incubated with Hoechst 33258 for $10 \mathrm{~min}$. The slides were washed twice with PBS and dipped in water to remove salt precipitates. Mowiol-mounting media was spread over the glass slide and 50 x $36 \mathrm{~mm}$ glass coverslip was gently put over the sections. Confocal microscopic images of fixed cell samples were acquired with Leica DMIRE2 microscope with a 40x oil-immersion objective.

\subsubsection{Morphometry}

To analyze axon and dendrite morphology of cultured primary neurons, individual neurons were imaged randomly in a blinded-manner using a 20x objective of Nikon epifluorescence microscope with a CCD camera. The axon length or total dendrite lengths were traced and measured manually using NIS element software (Nikon) or ImageJ software. Statistical tests ( $t$-test/ANOVA) were performed using GraphPad Prism 5.0c software and the values were represented as mean \pm SEM.

For in vivo assays, laser scanning confocal microscope (Leica) was used to acquire images in Z-stack in a blinded-manner. The images were analyzed using Imaris software (Bitplane) for measurement of dendrite lengths in 3D and for neuronal migration by counting the fraction of cells in external granule layer (EGL), molecular layer (ML) and internal granule layer (IGL). Statistical test was performed using GraphPad Prism 5.0c software and the values were represented as mean \pm SEM.

\subsubsection{Statistical tests}

All statistical tests were performed using GraphPad Prism 5.0c software. For comparison of two groups unpaired $t$-test was used whereas for comparison of three or more groups one-way Analysis of Variance (ANOVA) followed by Bonferroni posthoc test was used. Two-way ANOVA was used to compare three or more groups with multiple parameters followed by Bonferroni post-hoc test. Errors are displayed as standard error of mean (SEM). 
A scientist is happy, not in resting on his attainments but in the steady acquisition of fresh knowledge.

Max Planck

\section{Results}

\subsection{FBXO31 is a centrosomal E3 ubiquitin ligase}

\subsubsection{Widespread expression of $F B X$ genes}

As a preliminary screen to identify the candidate F-box protein (FBX) for my study, I started by studying the expression of $F B X$ genes in mouse tissue. To determine the brain-specific or brain-enriched expression of $F B X$ genes, RT-PCR was performed from different tissues of the P20 mouse including cortex, cerebellum, hippocampus, heart, lung, liver, spleen and kidney. Among the sixty-nine known F-box proteins, I focused on the eighteen that show an expression in the brain according to the ALLEN brain atlas. RT-PCR profiling revealed a widespread expression pattern of the selected $F B X$ genes. Although, many $F B X$ genes were highly expressed in the brain (FBXO2, FBXO9, FBX018, FBX021, FBXO31, FBXW2, FBXW5, $F B X W 7, F B X L 11$ and FBXL19), none of them seemed to have brain-specific expression pattern (Figure 3.1).

\subsubsection{FBXO31 is brain-enriched F-box protein}

Among the above-mentioned F-box proteins, FBXO31 is shown to be highly expressed in the human brain (Kumar et al., 2005). Given that the expression of FBXO31 has not been verified in rats, I sought to characterize FBXO31 expression in various tissues including cortex, cerebellum, hippocampus, heart, lung, liver, spleen and kidney using quantitative RT-PCR. Together with my colleague Nicola Schwedhelm-Domeyer, I collected tissue samples from P6, P12 and 4-month-old 


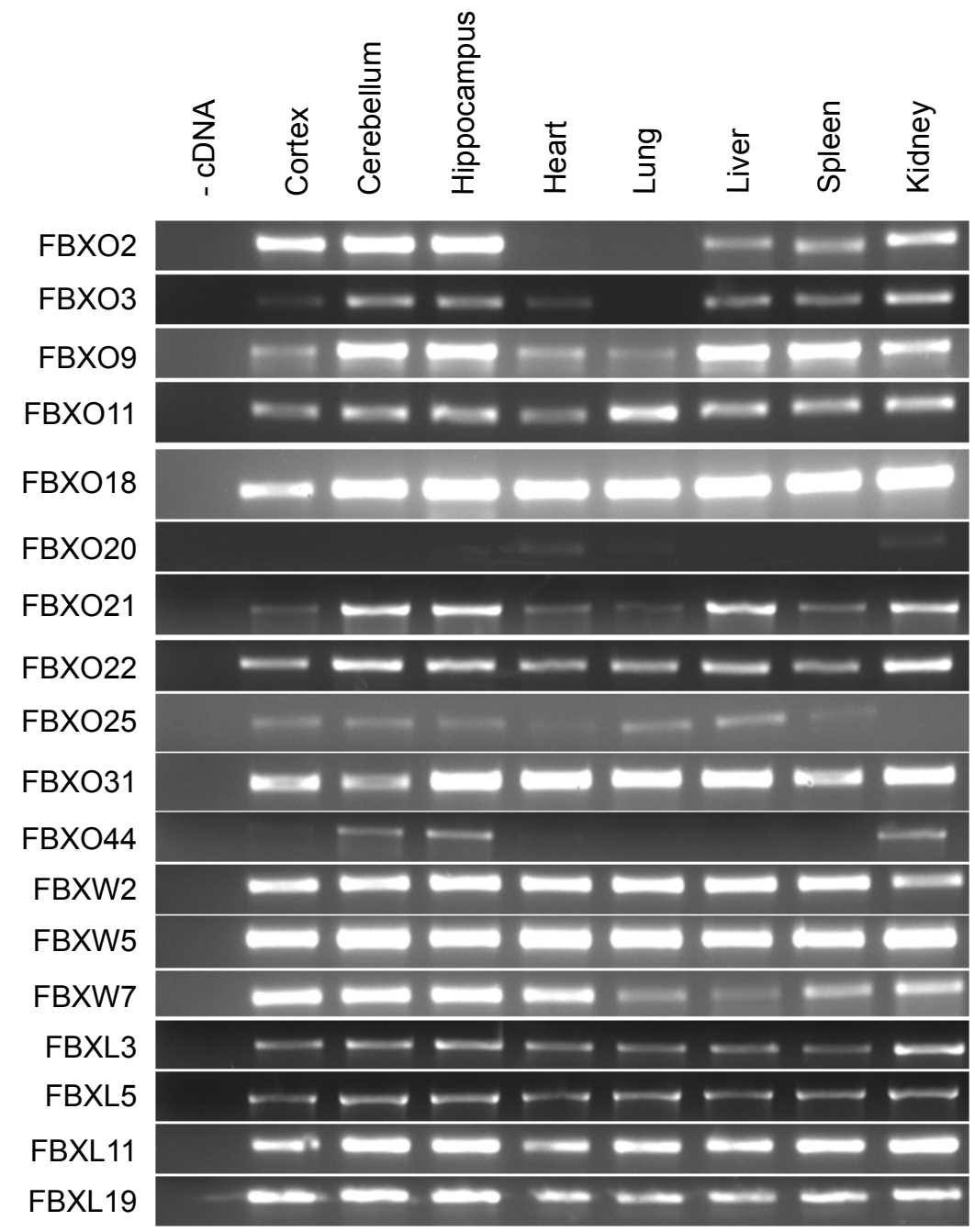

Figure 3.1: Expression of various $F B X$ genes in tissues of adult mouse. RT-PCR analysis of various $F B X$ genes in P20 mouse tissues including cortex, cerebellum, hippocampus, heart, lung, liver, spleen and kidney. The $F B X$ genes show widespread expression in different tissues isolated from P20 mouse. RNA from indicated tissues was reverse-transcribed into cDNA, which was subjected to PCR.

adult rats and subjected them to qRT-PCR. Consistent with the previous data, I found FBXO31 expression to be relatively enriched in brain as compared to the other tissues analyzed (Figure 3.2). 
A

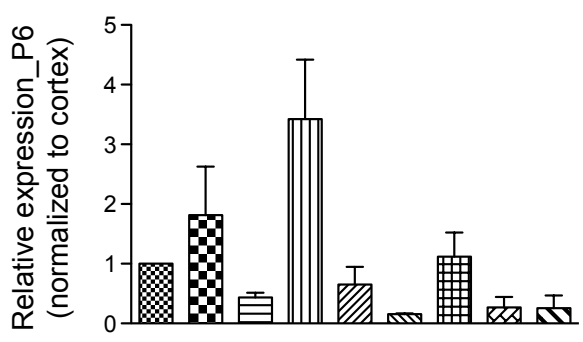

B

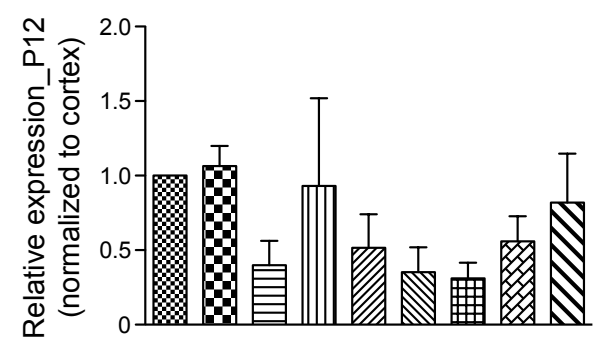

C

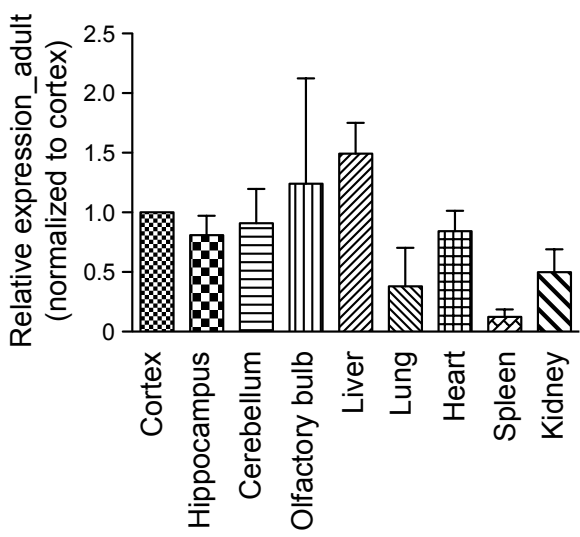

Figure 3.2: Quantitative PCR analysis of $F B X O 31$ expression in rat tissues. $q P C R$ analysis of $F B X O 31$ expression in various tissues of $\mathrm{P} 6$ rat pup (A), P12 rat pup (B) and 4-month-old adult rat (C). Data was normalized to $\beta$-actin and the values indicated are relative to cortex for each group. Nicola Schwedhelm-Domeyer and I isolated the tissues, extracted the RNA and synthesized the cDNA. Nicola Schwedhelm-Domeyer performed the qPCR.

\subsubsection{FBXO31 is localized at the centrosome}

To gain insight into the functions of FBXO31 in the nervous system, I first characterized its sub-cellular localization in neurons. I subjected the cultured cerebellar granule neurons and hippocampal neurons as well as HEK 293T cells to immunocytochemistry with the FBXO31 antibody. I found FBXO31 to be localized 


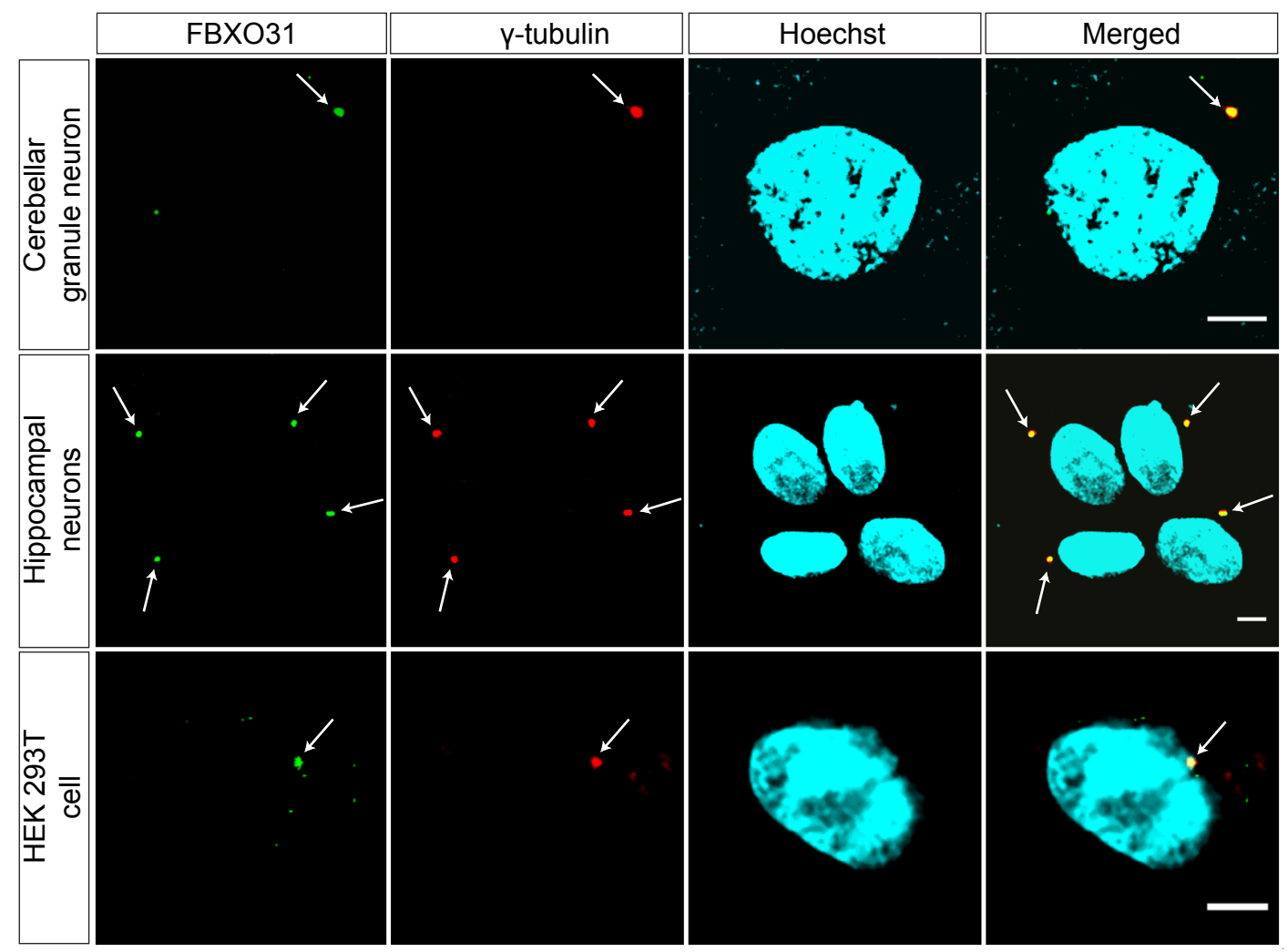

Figure 3.3: FBXO31 is localized at the centrosome. Cultured cerebellar granule neurons, hippocampal neurons and HEK 293T cells were immunostained with anti-FBXO31 and anti- $\gamma$-tubulin antibodies. The cells were counterstained with the DNA dye bis-benzamide Hoechst 33258 . Arrows indicate centrosome. Scale bar equals $5 \mu \mathrm{m}$.

at the centrosome. This finding was confirmed by co-localization analysis with the centrosomal marker $\gamma$-tubulin (Figure 3.3).

To confirm the specificity of the FBXO31 staining, I generated a recombinant FBXO31 protein fragment encoding amino acids 408-507 of the mouse FBXO31 against which the commercially available anti-FBXO31 antibody was generated. I verified the antigen-antibody interaction by immunoblotting and found a specific band at the expected size (Figure 3.4A). I further confirmed the specificity of the immunostaining in hippocampal neurons by antigen-antibody competition, whereby the antibody was pre-incubated with the antigen for $1 \mathrm{hr}$ prior to immunostaining 


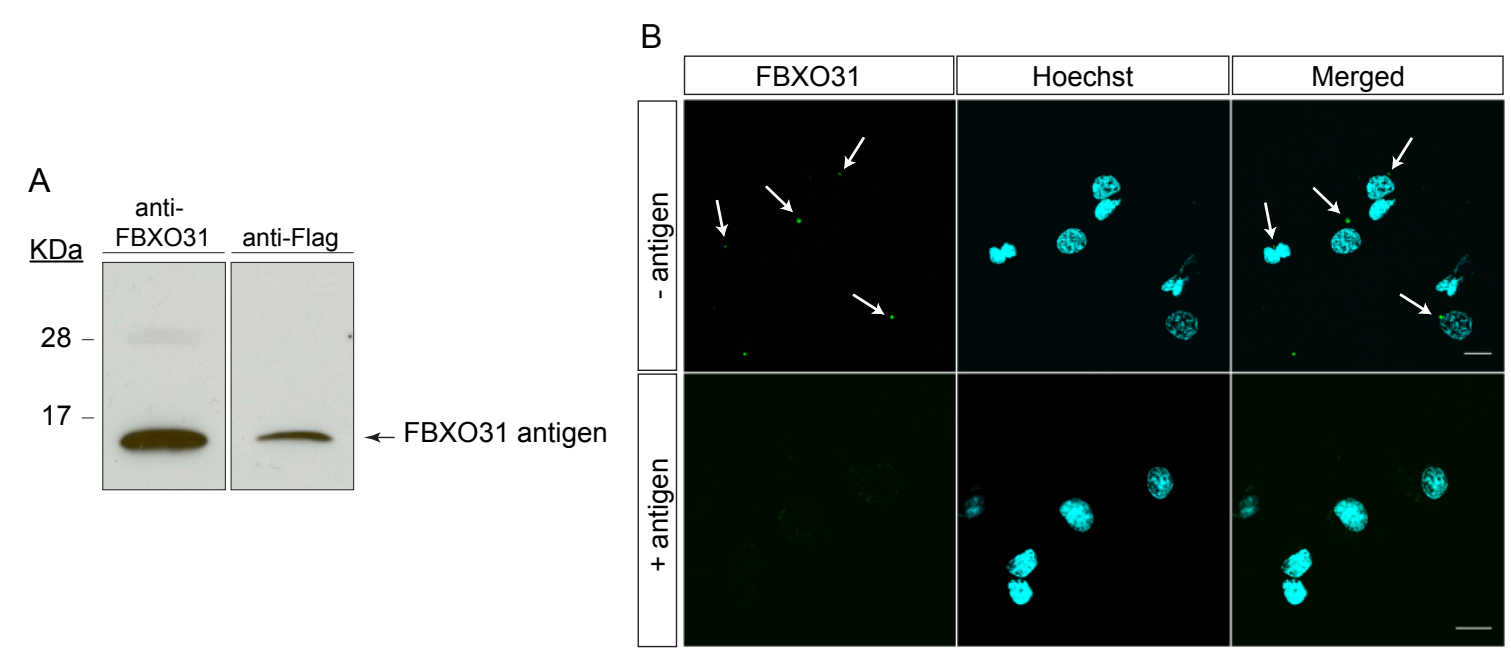

Figure 3.4: Validation of FBXO31 antibody. (A) $500 \mathrm{ng}$ of recombinant Flag-FBXO31 408507 , purified from bacteria was validated by immunoblotting with anti-FBXO31 and anti-Flag antibody. FBXO31 antigen was detected at expected size of $11 \mathrm{kDa}$. (B) Hippocampal neurons cultured from E18 rat embryos were immunostained with anti-FBXO31 antibody with or without pre-incubation with recombinant FBXO31 antigen 408-507. Arrows indicate centrosome. Scale bar equals $10 \mu \mathrm{m}$. The FBXO31 antigen was purified by Nicola Schwedhelm-Domeyer.

of the neurons. Using antigen-antibody competition, I could no longer detect the centrosomal localization of FBXO31 (Figure 3.4B), confirming FBXO31 as a centrosomal protein.

To determine the centrosomal-targeting region in FBXO31, I generated various GFP-tagged deletion mutants of FBXO31 along with my colleague Nicola Schwedhelm-Domeyer (Figure 3.5). I transfected the HEK $293 \mathrm{~T}$ cells with these deletion mutants together with a plasmid encoding a predominant-centrosomal protein DISC1 and subjected the cells to immunostaining. I found that while FBXO31 $\mathrm{WT}, \Delta \mathrm{F}, \Delta 1, \Delta 2$ and $\Delta 4$ co-localizes with DISC1 at the centrosome, FBXO31 $\Delta 3$ localizes to the nucleus and FBXO31 $\Delta 5$ freely diffuses in the cytoplasm (Figure 3.6). Therefore, I concluded that the region comprising of amino acid 60-274 in FBXO31 is responsible for its centrosomal localization. 


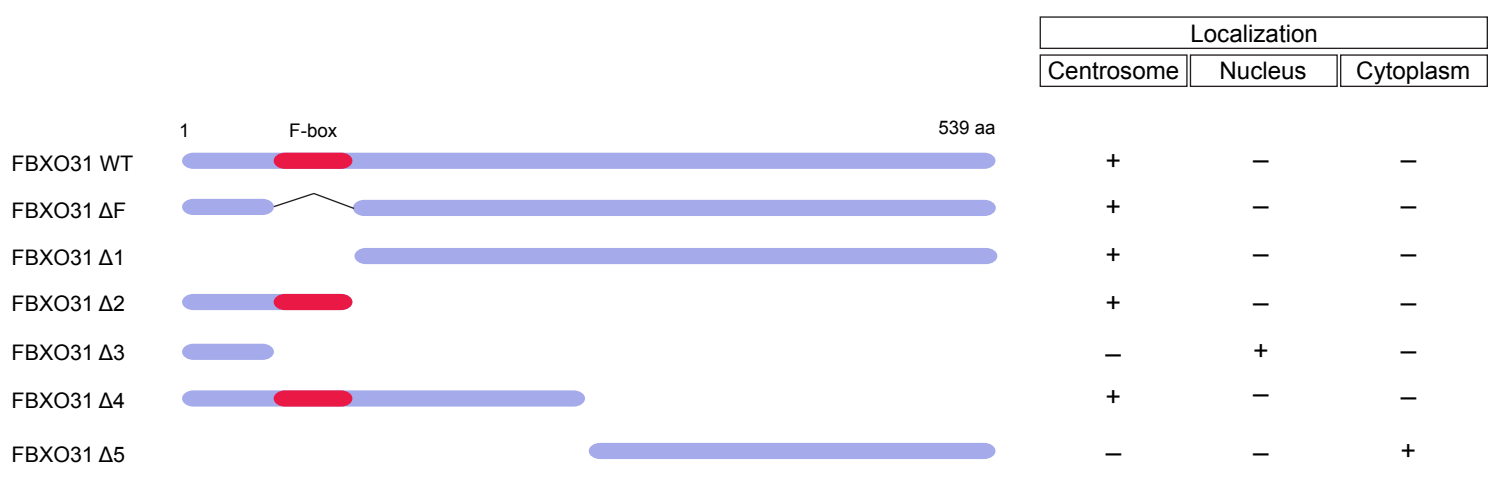

Figure 3.5: FBXO31 deletion mutants and their localization. Schematic representation of deletion mutants of FBXO31 evaluated for their centrosomal localization.

\subsection{Functional characterization of F-box protein FBXO31 in the nervous system}

FBXO31 has been previously reported to play a role in the regulation of cell cycle by inducing the degradation of cyclin D1 and hence mediating the transition from G1 to S phase (Kumar et al., 2005; Santra et al., 2009). Although, FBXO31 is enriched in the brain (Kumar et al., 2005), its function in the nervous system remains elusive.

In this study, I sought to investigate the function of FBXO31 in the nervous system. Since our lab specializes in studying cell-intrinsic programs that regulate axon growth, I addressed the function of FBXO31 in axon and dendrite growth regulation in neurons by loss-of-function and gain-of-function approaches.

\subsubsection{FBXO31 loss-of-function inhibits axon and dendrite growth in cerebellar granule neurons}

\section{Validation of FBXO31 RNAi constructs}

To investigate the role of FBXO31 in the neurons, I used an RNAi approach and generated three short-hairpin RNAi plasmids directed against different regions of FBXO31. The target sequences were homologous in human, rat and mouse 


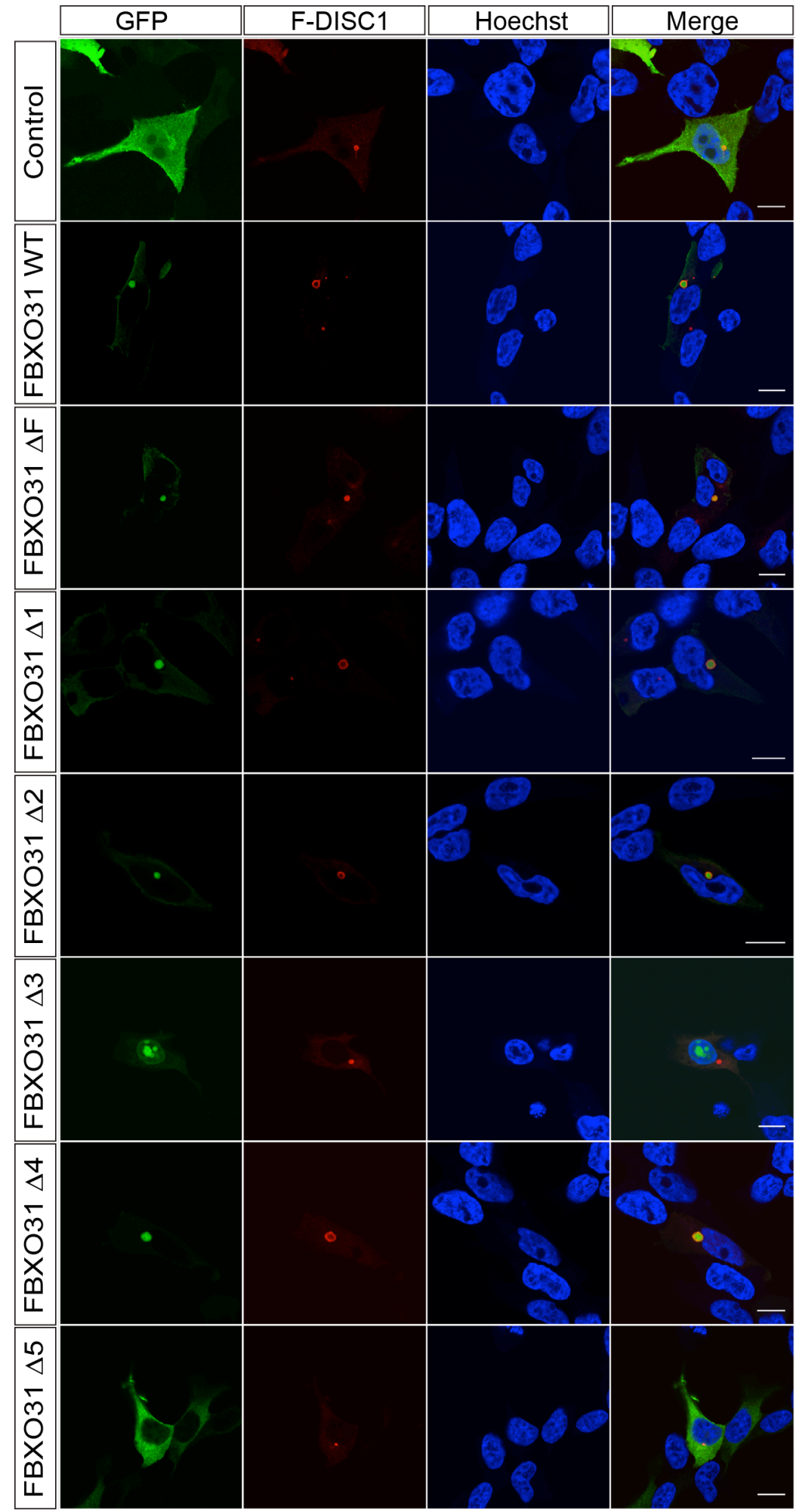

Figure 3.6: $60-274$ aa region is required for centrosomal localization of FBXO31. HEK 293T cells transfected with Flag-DISC1 and various GFP-FBXO31 deletion mutants were immunostained with anti-Flag and anti-GFP antibodies. The cells were counterstained with Hoechst 33258. Scale bar equals $10 \mu \mathrm{m}$. 


$\begin{array}{llllll} & 1 & \text { F-box } & & & 1620 \mathrm{bp} \\ \text { FВХО31 } & & \overline{\# 1} & \overline{\# 2} & \overline{\# 3}\end{array}$

\begin{tabular}{|c|c|c|c|c|c|c|c|c|}
\hline amin & $\mathrm{o}$ acids & $\mathrm{K}$ & D & $E$ & $\mathrm{~F}$ & $S$ & K & C \\
\hline to & & $5 \ldots G$ & & & & & & \\
\hline rat & bp & $5^{\prime} \ldots \mathrm{GAGG}$ & GAT & GA & STTC & TTCC & ACCA & TGT \\
\hline $\mathrm{mol}$ & $4 \mathrm{~h}$ & $5 ' \ldots G A G G$ & GAC & SGAC & STTC & ETCC & AACCAAG & TTG \\
\hline
\end{tabular}

FBXO31 RNAi\#1

GGATGAGTTCTCCACCAAGT

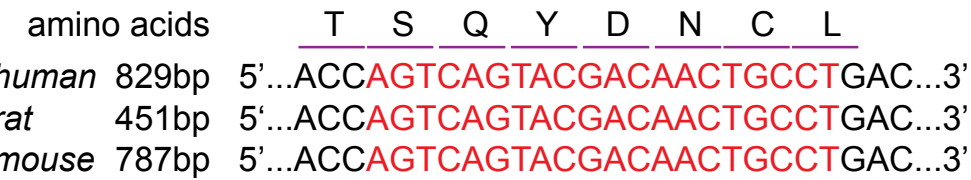

FBXO31 RNAI\#2 AGTCAGTACGACAACTGCCT

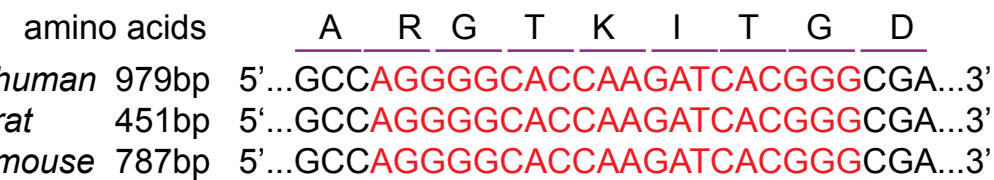

FBXO31 RNAi\#3 AGGGGCACCAAGATCACGGG

Figure 3.7: Targeting regions of FBXO31 short-hairpin RNAs. Short-hairpin FBXO31 RNAi were generated targeting three different regions of $F B X O 31$ gene (indicated as green line). The target sequences were homologous in human, rat and mouse.

FBXO31 sequences (Figure 3.7). To validate the FBXO31 RNAi constructs (\#1, \#2 and \#3), HEK 293T cells were transfected with either the empty control vector or with FBXO31 RNAi construct together with myc-tagged FBXO31 expression plasmid. The cells were lysed after 4 days and the cell lysates were subjected to immunoblotting with the myc antibody. While FBXO31 levels were not altered with FBXO31 RNAi\#2, the levels of FBXO31 were significantly reduced with FBXO31 RNAi\#1 and \#3 as compared to control (Figure 3.8). 


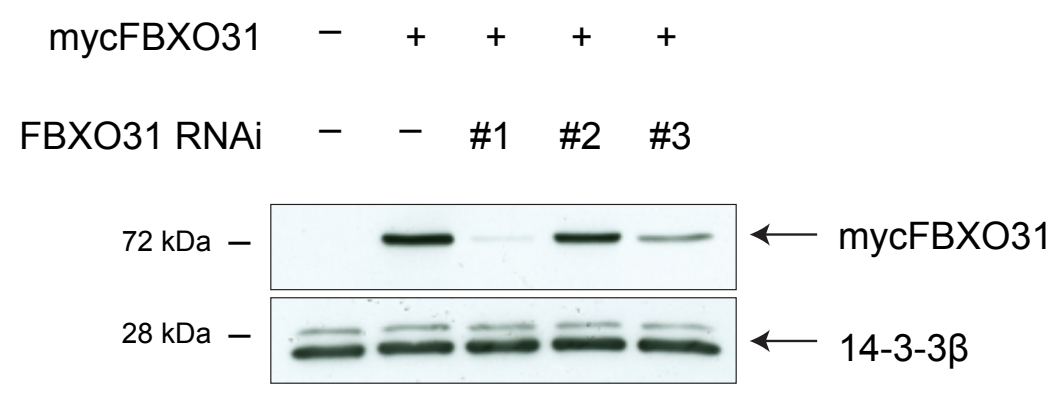

Figure 3.8: Validation of FBXO31 RNAi constructs. Lysates from HEK 293T cells, transfected with mycFBXO31 together with control or FBXO31 RNAi \#1, \#2 or \#3 plasmids, were probed with anti-myc antibody. 14-3-3 $\beta$ served as a loading control.

FBXO31 knockdown reduces axon and dendrite growth in cerebellar granule neurons

As a first approach to characterize the function of FBXO31 in neurons, I triggered FBXO31 knockdown in neurons and assessed them for changes in morphology. Cerebellar granule neurons were transfected $8 \mathrm{hrs}$ after plating with control vector or FBXO31 RNAi plasmids together with Bcl- $\mathrm{x}_{\mathrm{L}}$ and GFP-encoding plasmids. While GFP served as a marker for transfected neurons, Bcl- $\mathrm{x}_{\mathrm{L}}$, a Bcl-2 family member, inhibits neuronal apoptosis. Both GFP and $\mathrm{Bcl}-\mathrm{x}_{\mathrm{L}}$ have been shown to have no effect on neuronal morphology in cultured neurons (Konishi et al., 2004). The neurons were fixed at DIV 4 and analyzed for axon and dendrite growth as described in materials and methods. I found that there was a significant reduction of axon and dendrite lengths with FBXO31 RNAi\#1 and \#3 as compared to control. The axon and dendrite lengths of neurons transfected with FBXO31 RNAi\#2 did not change as compared to control-transfected neurons (Figure 3.9). 
A

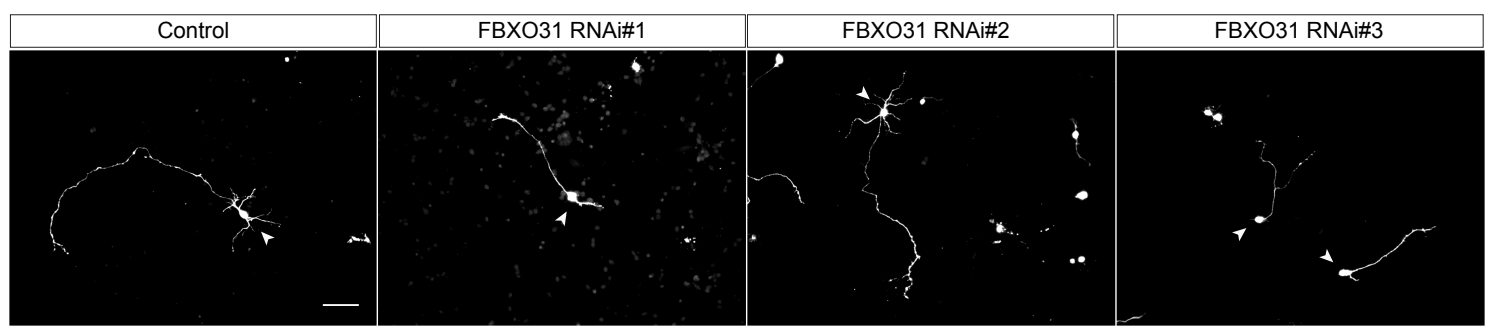

B

C
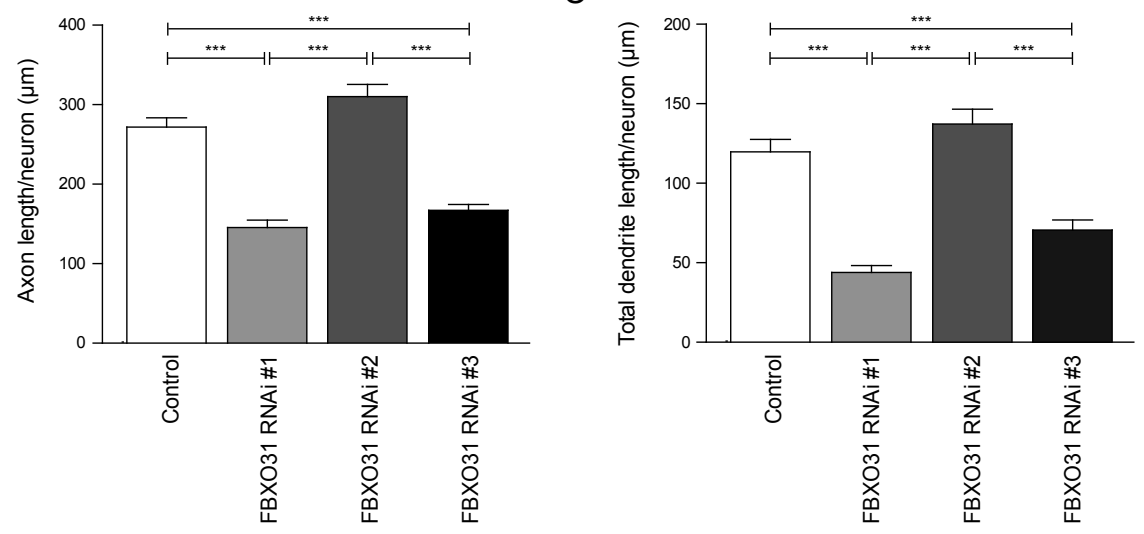

Figure 3.9: FBXO31 knockdown reduces axon and dendrite growth in cerebellar granule neurons. (A) Representative images of cerebellar granule neurons transfected with control, FBXO31 RNAi\#1, \#2 or \#3 plasmids together with GFP and Bcl- $x_{L}$ plasmids at DIV 0 and analyzed at DIV 4. Arrowheads indicate cell bodies. Scale bar equals $50 \mu \mathrm{m}$. (B) Quantification of axon lengths of granule neurons as shown in (A). A total of 539 cells were analyzed in 3 independent experiments. Control: 272.0 $\pm 11.57 \mu \mathrm{m}$; FBXO31 RNAi\#1: $145.7 \pm 9.18 \mu \mathrm{m}$; FBXO31 RNAi\#2: $310.1 \pm 15.43 \mu \mathrm{m}$; FBXO31 RNAi\#3: $167.1 \pm 7.28 \mu \mathrm{m}$. Values indicate mean \pm SEM (one-way ANOVA, ${ }^{* *} p<0.001$ ). (C) Quantification of total dendrite lengths of granule neurons as shown in (A). A total of 526 neurons were analyzed in 3 independent sets of experiments. Control: $119.7 \pm 7.96 \mu \mathrm{m}$, FBXO31 RNAi\#1: 43.97 $\pm 4.19 \mu \mathrm{m}$, FBXO31 RNAi\#2: $137.2 \pm 9.41 \mu \mathrm{m}$, FBXO31 RNAi\#3: 70.56 $\pm 6.3 \mu \mathrm{m}$. Values indicate mean \pm SEM (one-way ANOVA, ${ }^{* * *} \mathrm{p}<0.001$ ).

Further, to rule out the off-target effects of RNAi on neuronal morphology, I generated a myc-tagged FBXO31 rescue mutant (FBXO31-Res) that is resistant to RNAi by introducing silent mutations into the FBXO31 targeting region (Figure 3.10A). To validate the FBXO31 rescue mutants, HEK 293T cells were transfected with wild-type FBXO31 or FBXO31-Res plasmids together with FBXO31 RNAi\#1 
plasmid. The cells were lysed four days after transfection and the lysates were subjected to immunoblotting. As demonstrated previously, there was a significant decrease in FBXO31 WT levels upon FBXO31 knockdown but the levels of FBXO31Res did not change upon expression of FBXO31 RNAi plasmid, thereby confirming that FBXO31-Res is resistant to RNAi-mediated degradation (Figure 3.10B).

A

FBX031 RNAi\#1

FBXO31-Res

\section{$\frac{D}{G \text { GAT GAG TTC TCC }} \frac{\mathrm{E}}{\mathrm{ACC}} \frac{\mathrm{F}}{\mathrm{AAG} T}$}

5' C CAG ATT GTG AAG AAG GAT GAA TTT TCAACA AAG TGC AAC CAG ACG G 3'

B

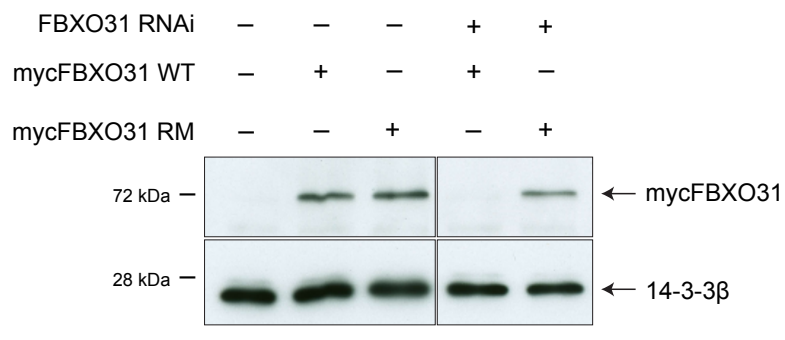

Figure 3.10: Generation and validation of FBXO31-Res plasmid. (A) Using site-directed mutagenesis, silent mutations were introduced into the FBXO31 RNAi\#1 targeting region to generate FBXO31-Res plasmid. (B) HEK 293T cells were transfected with control, mycFBXO31 WT or mycFBXO31-Res expression plasmids together with FBXO31 RNAi\#1 or control plasmids. Cell lysates were immunoblotted for FBXO31 with anti-myc antibody. 14-3-3 $\beta$ served as a loading control.

Having generated the FBXO31-Res plasmid, I carried out the rescue experiments in which I transfected the neurons with either control or FBXO31 RNAi plasmid together with FBXO31-Res expression plasmid at DIV 0. The neurons were assessed for axon and dendrite growth at DIV 4. As observed previously, there was a significant decrease in axon and dendrite growth upon FBXO31 knockdown, which was partially rescued upon expression of FBXO31-Res (Figure 3.11).

To examine whether FBXO31 knockdown affects neuronal survival, my colleague Chaitali Mukherjee transfected the neurons at DIV 2 with empty vector control or FBXO31 RNAi plasmids together with transfection marker $\beta$-galactosidase 
A

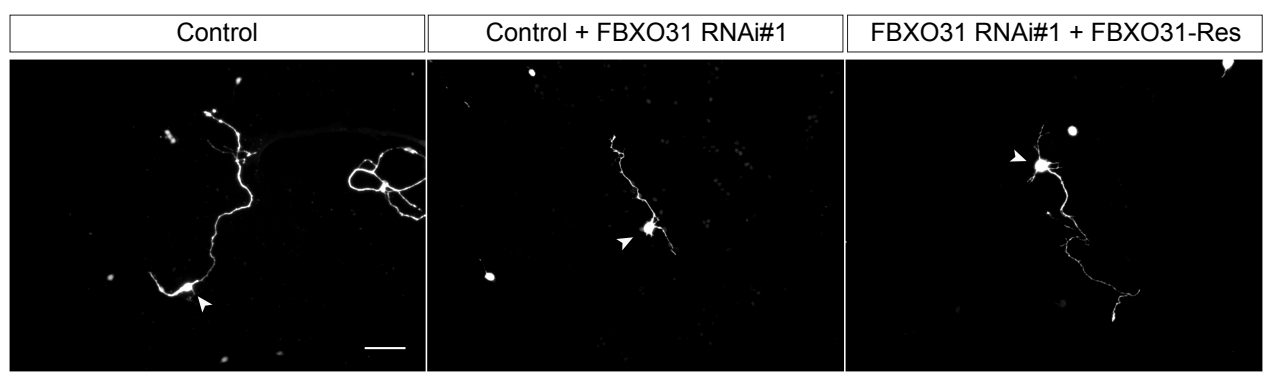

$\mathrm{B}$

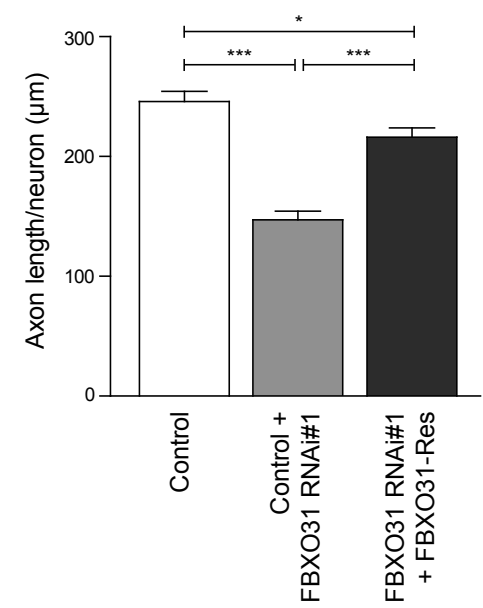

C

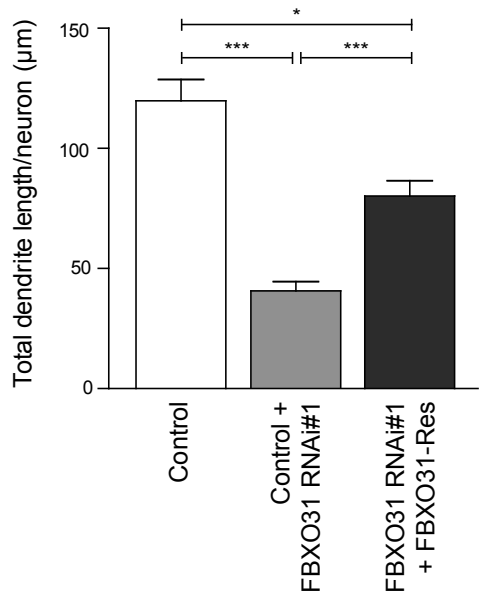

Figure 3.11: FBX031-Res reverses the FBXO31 RNAi phenotype on axon and dendrite growth in cerebellar granule neurons. (A) Representative images of cerebellar granule neurons transfected with control, FBXO31 RNAi\#1 or FBXO31 RNAi\#1 and mycFBXO31-Res plasmids together with GFP and Bcl- $X_{L}$ expression plasmids at DIV 0 and analyzed at DIV 4. Arrowheads indicate cell bodies. Scale bar equals $50 \mu \mathrm{m}$. (B) Quantification of axon lengths of granule neurons as shown in (A). A total of 296 cells were analyzed in 3 independent sets of experiments. Control: 246.0 $\pm 8.3 \mu \mathrm{m}$; FBXO31 RNAi\#1: $147.1 \pm 7.33 \mu \mathrm{m}$, FBXO31 RNAi\#1+FBXO31-Res: 216.1 1 7.93 $\mu \mathrm{m}$. Values indicate mean \pm SEM (one-way ANOVA, ${ }^{*} \mathrm{p}<0.05$, ${ }^{* * *} \mathrm{p}<0.001$ ). (C) Quantification of total dendrite lengths of granule neurons as shown in (A). A total of 291 cells were analyzed in 3 independent sets of experiments. Control: 119.8 $\pm 8.91 \mu \mathrm{m}$, FBXO31 RNAi\#1: 40.65 \pm 3.89 $\mu \mathrm{m}, F B X O 31$ RNAi\#1 + FBXO31-Res: $80.17 \pm 6.43 \mu \mathrm{m}$. Values indicate mean \pm SEM (one-way ANOVA, $\left.{ }^{* * *} p<0.001\right)$.

but without the Bcl- $\mathrm{x}_{\mathrm{L}}$ expression plasmid. Neurons were fixed at DIV 6 and assessed for apoptotic neurons characterized by fragmented axons and pyknotic nucleus. Chaitali Mukherjee found that while FBXO31 RNAi\#1 and \#2 (non- 
functional) slightly increase neuronal apoptosis, FBXO31 RNAi\#3 did not show any change as compared to control neurons (Figure 3.12), suggesting a rather subordinate role of FBXO31 in neuronal survival.

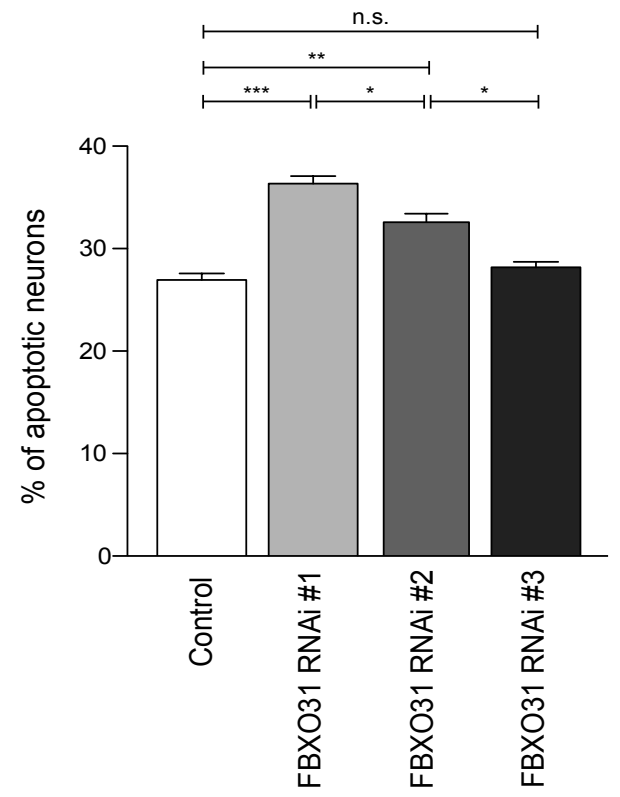

Figure 3.12: FBXO31 regulates neuronal survival. Cerebellar granule neurons were transfected with control, FBXO31 RNAi\#1, \#2 or \#3 plasmid together with $\beta$-gal expression plasmid at DIV 2 and analyzed for neuronal survival at DIV 6. A total of 1585 cells were analyzed. Percentage of apoptotic neurons - Control: 26.93 \pm 0.63 ; FBXO31 RNAi\#1: $36.37 \pm 0.74$; FBXO31 RNAi\#2: 32.6 \pm 0.83 ; FBXO31 RNAi\#3: 28.19 \pm 0.53 . Values indicate mean \pm SEM (one-way ANOVA, ${ }^{*} \mathrm{p}<0.05,{ }^{* *} \mathrm{p}<0.01,{ }^{* * *} \mathrm{p}<0.001$, n.s. $=$ not significant). The survival assay was performed by Chaitali Mukherjee.

\subsubsection{FBXO31 gain-of-function promotes axon and dendrite growth in cerebellar granule neurons}

Besides the loss-of-function approach to assess the role of FBXO31 on axon and dendrite growth, I asked whether FBXO31 overexpression would result in the opposite effect on axons and dendrites. In order to test this, I transfected the neurons with the mycFBXO31 expression plasmid or with corresponding empty 
vector control at DIV 0 and analyzed the neurons three days later for their axon and dendrite lengths. I found that overexpression of FBXO31 significantly increases axon and dendrite length (Figure 3.13).
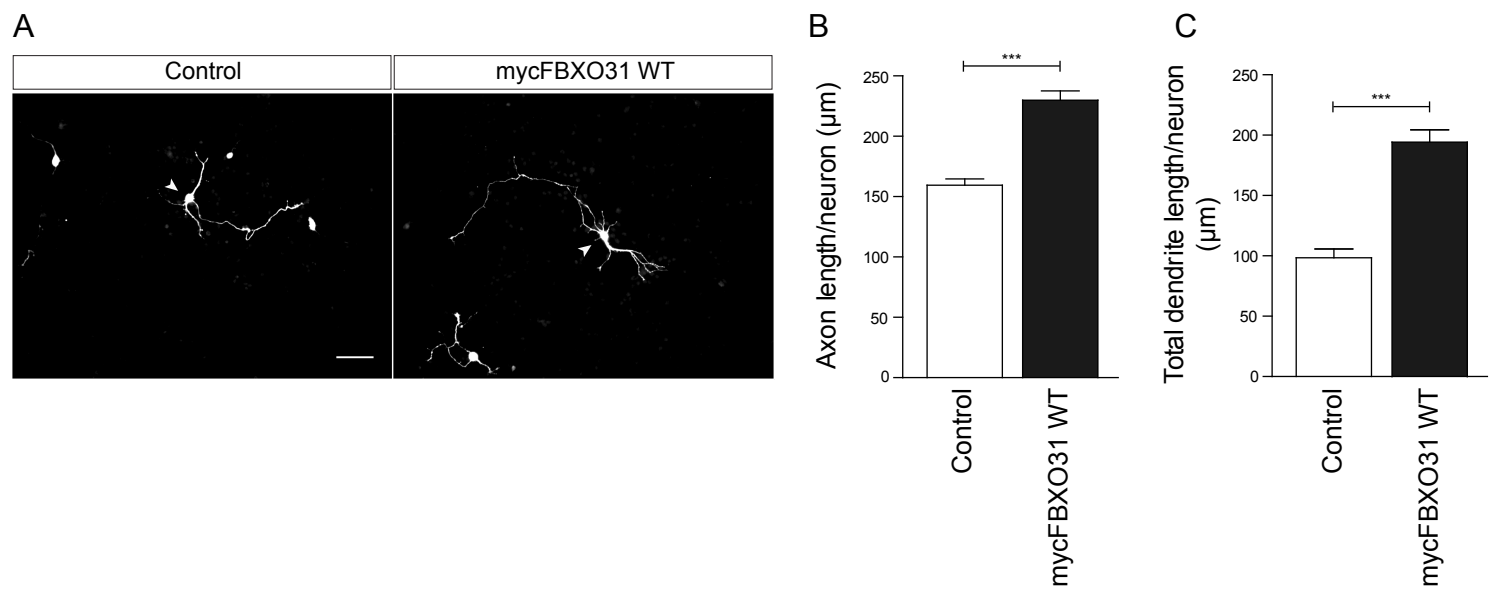

Figure 3.13: FBXO31 overexpression promotes axon and dendrite growth in cerebellar granule neurons. (A) Representative images of cerebellar granule neurons transfected with control or myc FBXO31 plasmids together with GFP and Bcl- $\mathrm{XL}_{\mathrm{L}}$ at DIV 0 and analyzed at DIV 3. Arrowheads indicate cell bodies. Scale bar equals $50 \mu \mathrm{m}$. (B) Quantification of axon lengths of granule neurons as shown in (A). A total of 274 neurons were analyzed in 3 independent sets of experiments. Control: 159.5 $\pm 5.1 \mu \mathrm{m}$ and mycFBXO31 WT: 229.9 $\pm 7.69 \mu \mathrm{m}$. Values indicate mean \pm SEM (unpaired $t$-test, ${ }^{* * *} \mathrm{p}<0.001$ ). (C) Quantification of total dendrite lengths of granule neurons as shown in (A). A total of 261 neurons were analyzed in 3 independent sets of experiments. Control: $98.37 \pm 7.28 \mu \mathrm{m}$ and mycFBXO31 WT: $194.5 \pm 10.11 \mu \mathrm{m}$. Values indicate mean \pm SEM (unpaired $t$-test, ${ }^{* * *} p<0.001$ ).

F-box proteins are known to form functional ubiquitin ligase complex by associating with their partners Skp1 and Cul1 via the highly conserved F-box domain (Kipreos and Pagano, 2000). FBXO31 has been previously reported to associate with Cul1 and Skp1 through its F-box domain (Kumar et al., 2005). I asked if the association of FBXO31 with Skp1 and Cul1 and thus its ligase activity is required for its effect on axon and dendrite growth in neurons. I used mycFBXO31 $\Delta \mathrm{F}$ construct (generously provided by Dr. Raman Kumar and Prof. David Callen, University of Adelaide, Australia) in which F-box domain has been deleted thereby disrupting its 
association with Skp1 and Cul1 (Kumar et al., 2005). To confirm the specificity of FBXO31 $\Delta$ F mutant, I transfected HEK 293T cells with GFP-FBXO31 WT or $\Delta \mathrm{F}$ plasmids together with mycSkp1 plasmid and respective control vectors. The cell lysates were subjected to immunoprecipitation with anti-GFP antibody and immunoblotted for Skp1 and Cul1. While I observed an interaction of FBXO31 WT with Skp1 and Cul1, this interaction was lost with FBXO31 $\Delta F$ mutant (Figure 3.14), suggesting that FBXO31 $\Delta \mathrm{F}$ is ligase-dead mutant.

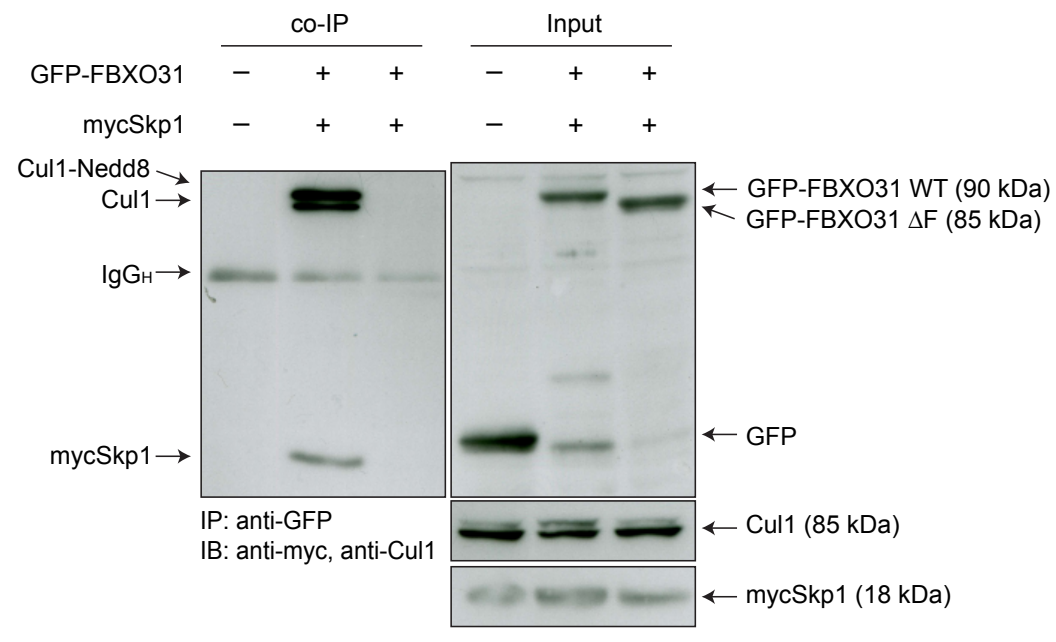

Figure 3.14: FBXO31 $\Delta \mathrm{F}$ does not form a functional SCF complex. HEK $293 \mathrm{~T}$ cells were transfected with GFP-FBXO31 WT or $\Delta \mathrm{F}$ plasmids together with mycSkp1 and respective control vectors. The cell lysates were subjected to immunoprecipitation with anti-GFP antibody and probed for Skp1 and Cul1.

Next, I transfected neurons at DIV 0 with either control or FBXO31 WT or FBXO31 $\Delta \mathrm{F}$ expression plasmid and analyzed them at DIV 3 for their axon and dendrite lengths. As previously observed, I found a significant increase in axon and dendrite length of the neurons expressing wild-type FBXO31, while there was no change in either the axon or the dendrite length of the neurons expressing FBXO31 $\Delta \mathrm{F}$ as compared to the control neurons (Figure 3.15). 
A

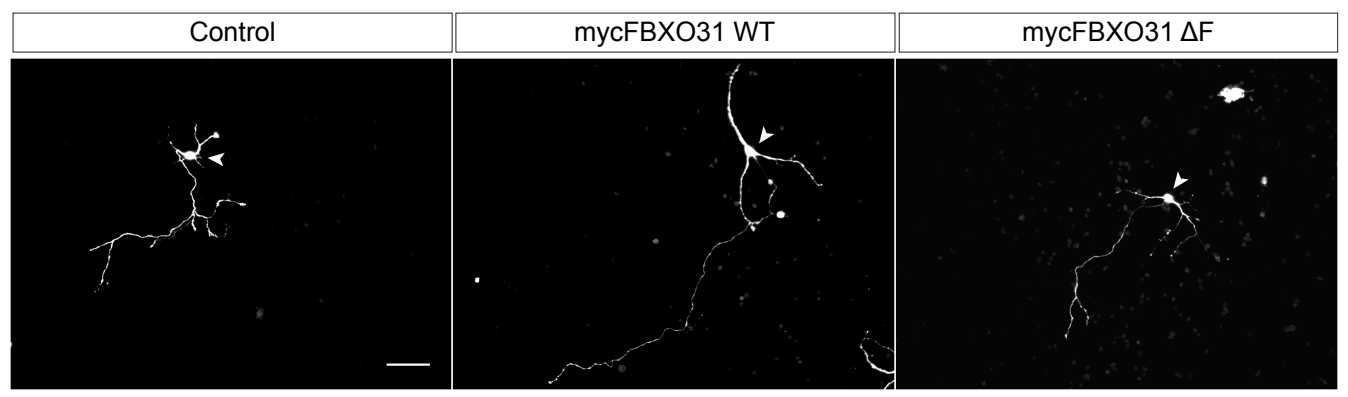

B

C
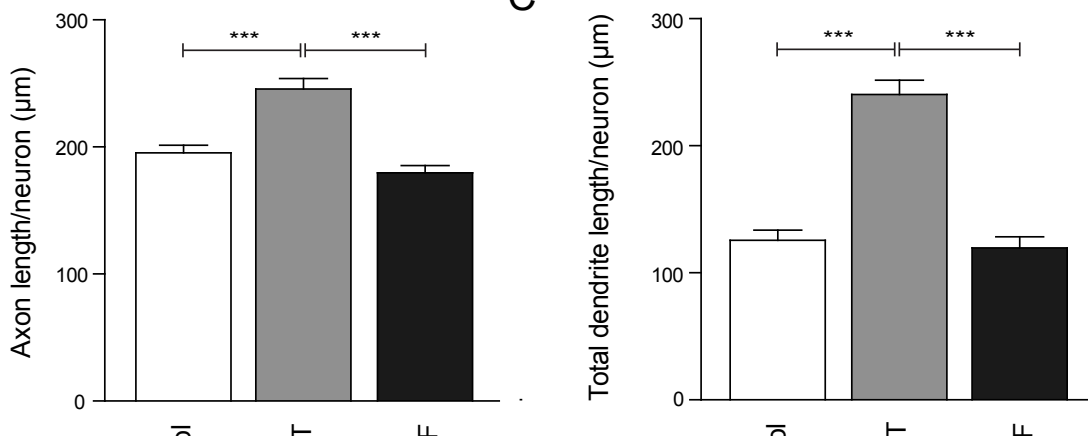

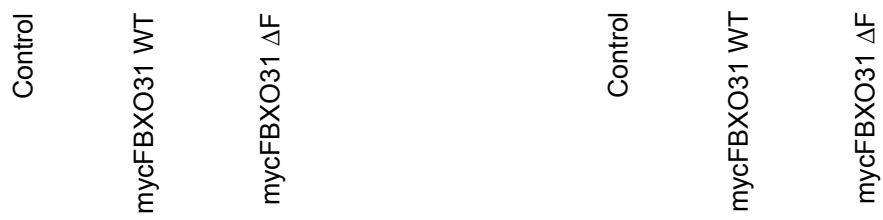

Figure 3.15: FBXO31-SCF ligase activity is essential for axon and dendrite growth in cerebellar granule neurons. (A) Representative images of cerebellar granule neurons transfected with control, mycFBXO31 WT or mycFBXO31 $\Delta \mathrm{F}$ together with GFP and Bcl- $\mathrm{x}_{\mathrm{L}}$ at DIV 0 and analyzed at DIV 3. Arrowheads indicate cell bodies. Scale bar equals $50 \mu \mathrm{m}$. (B) Quantification of axon lengths of granule neurons as shown in (A). A total of 381 neurons were analyzed in 3 independent sets of experiments. Control: $195.4 \pm 6.03 \mu \mathrm{m}$, mycFBXO31 WT: $245.6 \pm 8.17 \mu \mathrm{m}$ and mycFBXO31 $\triangle F: 179.5 \pm 5.72 \mu \mathrm{m}$. Values indicate mean \pm SEM (one-way ANOVA, ${ }^{* * *} \mathrm{p}<0.001$ ). (C) Quantification of total dendrite lengths of granule neurons as shown in (A). A total of 341 neurons were analyzed in 3 independent sets of experiments. Control: $125.7 \pm 7.94 \mu \mathrm{m}$, mycFBXO31 $W T: 240.4 \pm 11.07 \mu \mathrm{m}$ and mycFBXO31 $\Delta F: 119.7 \pm 8.63 \mu \mathrm{m}$. Values indicate mean \pm SEM (oneway ANOVA, $\left.{ }^{* * *} \mathrm{p}<0.001\right)$. 


\subsubsection{FBXO31 loss-of-function reduces axon and dendrite growth in hippocampal and cortical neurons}

To test whether the effect of FBXO31 on axon and dendrite growth is specific to CGNs or whether it is a general mechanism of axon and dendrite growth control in different neuronal subtypes, I used cultured hippocampal and cortical neurons isolated from E18 rat embryos. These neurons were transfected at DIV 1 with either empty vector control or FBXO31 RNAi\#1 plasmid. Neurons were analyzed for their axon and dendrite lengths at DIV 5. As observed with CGNs, I found a significant decrease in axon and dendrite growth in both hippocampal neurons (Figure 3.16AC) and cortical neurons (Figure 3.16D-F) upon FBXO31 knockdown as compared to control neurons, suggesting a general mechanism of FBXO31 effect on neuronal morphogenesis.

\subsubsection{FBXO31 regulates the establishment of axon-dendrite polarity in neurons}

The establishment of axon-dendrite polarity in neurons is a tightly regulated process. Besides extrinsic cues that regulate neuronal polarization, intrinsic cues particularly E3 ubiquitin ligases including Smurf1, Smurf2, Siah and TRIM32 have been reported to regulate neuronal polarization (Schwamborn et al., 2007, 2009; Famulski et al., 2010; Cheng et al., 2011). Therefore, I asked if FBXO31 plays a role in neuronal polarization.

\subsubsection{FBXO31 overexpression leads to a polarization defect in cere- bellar granule neurons}

While characterizing the function of FBXO31 in axon and dendrite growth in CGNs, I also observed FBXO31 knockdown neurons that are not polarized. Therefore, to test whether FBXO31 plays a role in neuronal polarization, I analyzed cerebellar granule neurons that were transfected with FBXO31 WT plasmid or con- 


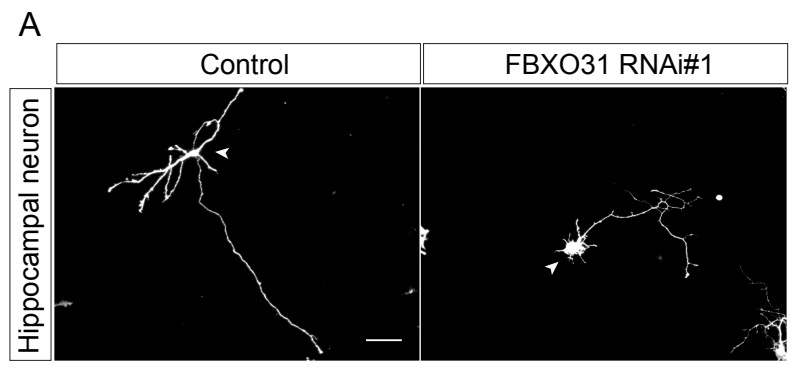

B
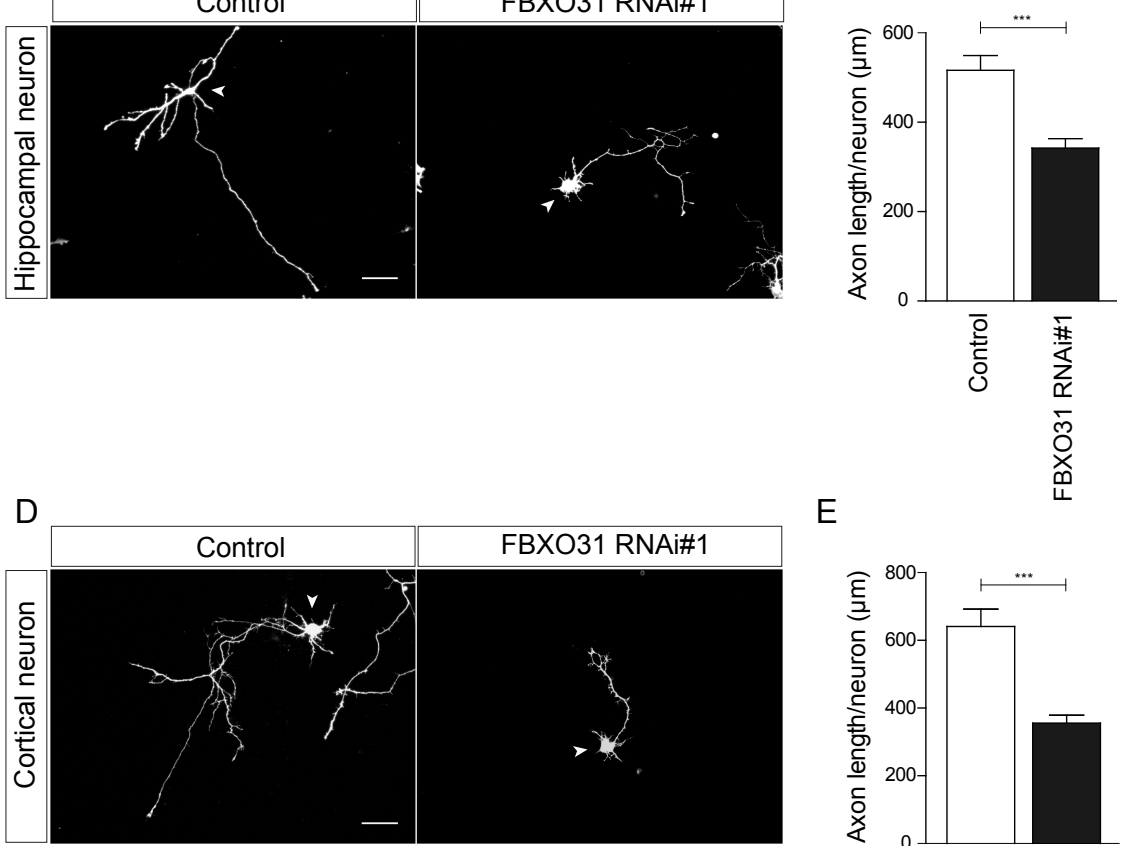

$\mathrm{E}$

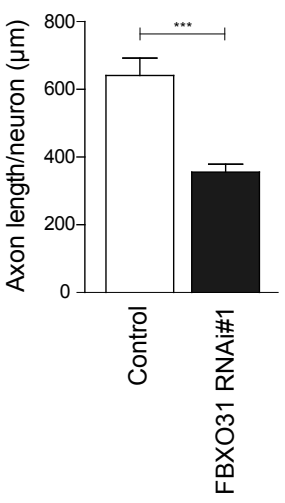

C
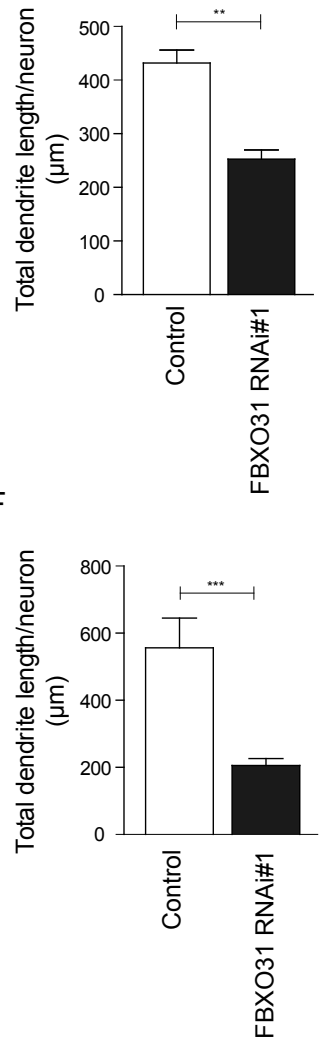

Figure 3.16: FBXO31 knockdown reduces axon and dendrite growth in hippocampal and cortical neurons. (A) Representative images of cultured hippocampal neurons transfected with control or bi-cistronic FBXO31 RNAi\#1/CMV-GFP plasmid together with Bcl- $\mathrm{X}_{\mathrm{L}}$ at DIV 1 and analyzed at DIV 5. Arrowheads indicate cell bodies. Scale bar equals $50 \mu \mathrm{m}$. (B) Quantification of axon lengths of hippocampal neurons as shown in (A). A total of 190 neurons were analyzed in 3 independent sets of experiments. Control: $516.1 \pm 33.29 \mu \mathrm{m}$ and FBXO31 RNAi\#1: $342.1 \pm 21.04$ $\mu \mathrm{m}$. Values indicate mean \pm SEM (unpaired $t$-test, ${ }^{* * *} \mathrm{p}<0.001$ ). (C) Quantification of total dendrite length of hippocampal neurons as shown in $(A)$. A total of 184 neurons were analyzed. Control: $431.8 \pm 24.35 \mu \mathrm{m}$ and FBXO31 RNAi\#1: $252.6 \pm 17.28 \mu \mathrm{m}$. Values indicate mean \pm SEM (unpaired $t$-test, $\left.{ }^{* * *} \mathrm{p}<0.001\right)$. (D) Representative images of cultured cortical neurons transfected with control or FBXO31 RNAi\#1 together with GFP and Bcl- $x_{\mathrm{L}}$ expression plasmids at DIV 1 and analyzed at DIV 5. Arrowheads indicate cell bodies. Scale bar equals $50 \mu \mathrm{m}$. (E) Quantification of axon lengths of cortical neurons as shown in (D). A total of 147 neurons were analyzed in 3 independent sets of experiments. Control: $640.7 \pm 51.54 \mu \mathrm{m}$ and FBXO31 RNAi\#1: $355.6 \pm 23.49 \mu \mathrm{m}$. Values indicate mean \pm SEM (unpaired $t$-test, ${ }^{* * *} \mathrm{p}<0.001$ ). (F) Quantification of total dendrite lengths of hippocampal neurons as shown in (D). A total of 164 neurons were analyzed in 3 independent sets of experiments. Control: $556.3 \pm 88.79 \mu \mathrm{m}$ and FBXO31 RNAi\#1: $205.7 \pm 20.35 \mu \mathrm{m}$. Values indicate mean \pm SEM (unpaired $t$-test, ${ }^{* * *} \mathrm{p}<0.001$ ). 
trol vector at DIV 0 and fixed at DIV 3. I measured the ratio of the longest to the second longest process length and defined neurons as non-polarized if the ratio of longest to second longest process length was less than 2 . While I found $18 \%$ of control neurons to be non-polarized, approximately $38 \%$ of FBXO31-overexpressing neurons were not polarized (Figure 3.17) .
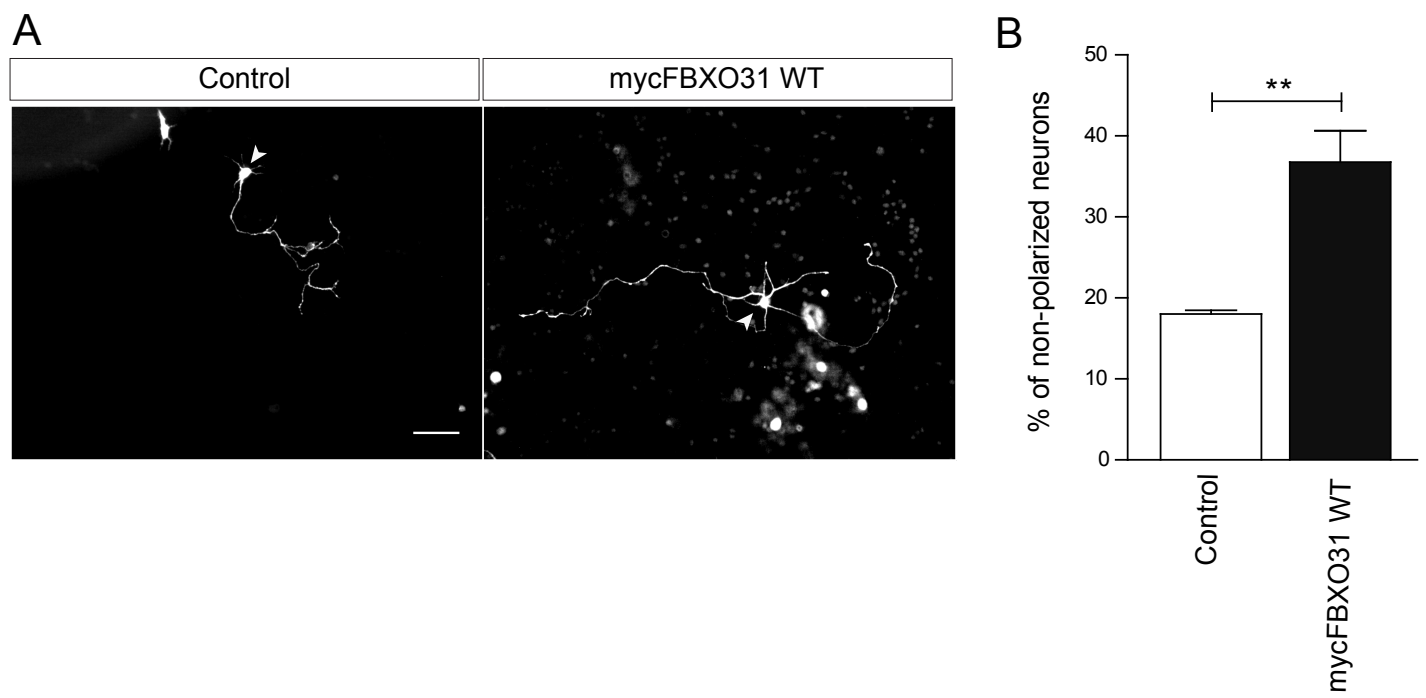

Figure 3.17: FBXO31 regulates neuronal polarization in cerebellar granule neurons. (A) Representative images of cerebellar granule neurons transfected with control or mycFBXO31 WT plasmid together with GFP and Bcl- $x_{\mathrm{L}}$ expression plasmids at DIV 0 and analyzed at DIV 3. The neurons were defined as polarized when the longest process was at least twice as long as the second longest process. Arrowheads indicate cell bodies. Scale bar equals $50 \mu \mathrm{m}$. (B) Quantification of percentage of non-polarized neurons as shown in (A). A total of 256 neurons were analyzed in 3 independent sets of experiments. Percentage of non-polarized neurons Control: $18.0 \pm 0.47$ and mycFBXO31 WT: $36.76 \pm 3.9$. Values indicate mean \pm SEM (unpaired $t$-test, $\left.{ }^{* *} \mathrm{p}<0.01\right)$.

\subsubsection{FBXO31 regulates neuronal polarity in hippocampal neurons}

Hippocampal neurons are well-established for studying neuronal polarization in vitro. Their development in culture has been divided into different stages based on the morphology as described in the introduction. I therefore took advantage of 
hippocampal neurons to ask if FBXO31 indeed has a role in neuronal polarization. Axons harbor the axon initial segment (AIS), which is characterized by the presence of AnkyrinG (AnkG). AnkG is responsible for organization of the AIS and thus maintenance of neuronal polarity. I transfected cultured hippocampal neurons with control, FBXO31 WT or FBXO31 $\Delta \mathrm{F}$ plasmids together with plasmids encoding GFP and Bcl- $x_{\mathrm{L}}$ at DIV 1. Neurons were fixed at DIV 7 and immunostained for GFP and AnkG. I observed a multiple-axon phenotype in neurons that were expressing FBXO31 WT but not in FBXO31 $\Delta \mathrm{F}$ or control conditions (Figure 3.18), suggesting that FBXO31 regulates neuronal polarity.

Besides the gain-of-function approach, I also asked if loss of FBXO31 in neurons would induce a no-axon phenotype. To test this hypothesis, I transfected hippocampal neurons with control vector or FBXO31 RNAi\#1 plasmid. The neurons were fixed at DIV 6 and immunostained for GFP and AnkG. I observed a no-axon phenotype upon knockdown of FBXO31 (Figure 3.19).

\subsubsection{FBXO31 promotes dendrite growth and neuronal mi- gration in developing cerebellum}

Cerebellar granule neurons are born in the external granule layer (EGL) of the cerebellum where they migrate tangentially as bipolar cells until they reach the molecular layer (ML). The cell body of the granule neurons then migrates radially into the internal granule layer (IGL) extending a bifurcating axon into the ML that forms, together with other axons, the parallel fiber bundle. Given that FBXO31 regulates CGN development in vitro, I asked if FBXO31 has a function in the developing cerebellum in vivo.

To address this, Dr Judith Stegmüller and I electroporated P4 rat pups with either bicistronic U6 $\phi / \mathrm{CMV}-\mathrm{GFP}$ control or FBXO31 RNAi\#1/CMV-GFP plasmids. The pups were sacrificed at P9 and the cerebella were analyzed for in vivo function of FBXO31. I analyzed the CGNs for their total dendrite length in 3D using Imaris software (Bitplane). As seen earlier in vitro, I found that FBXO31 


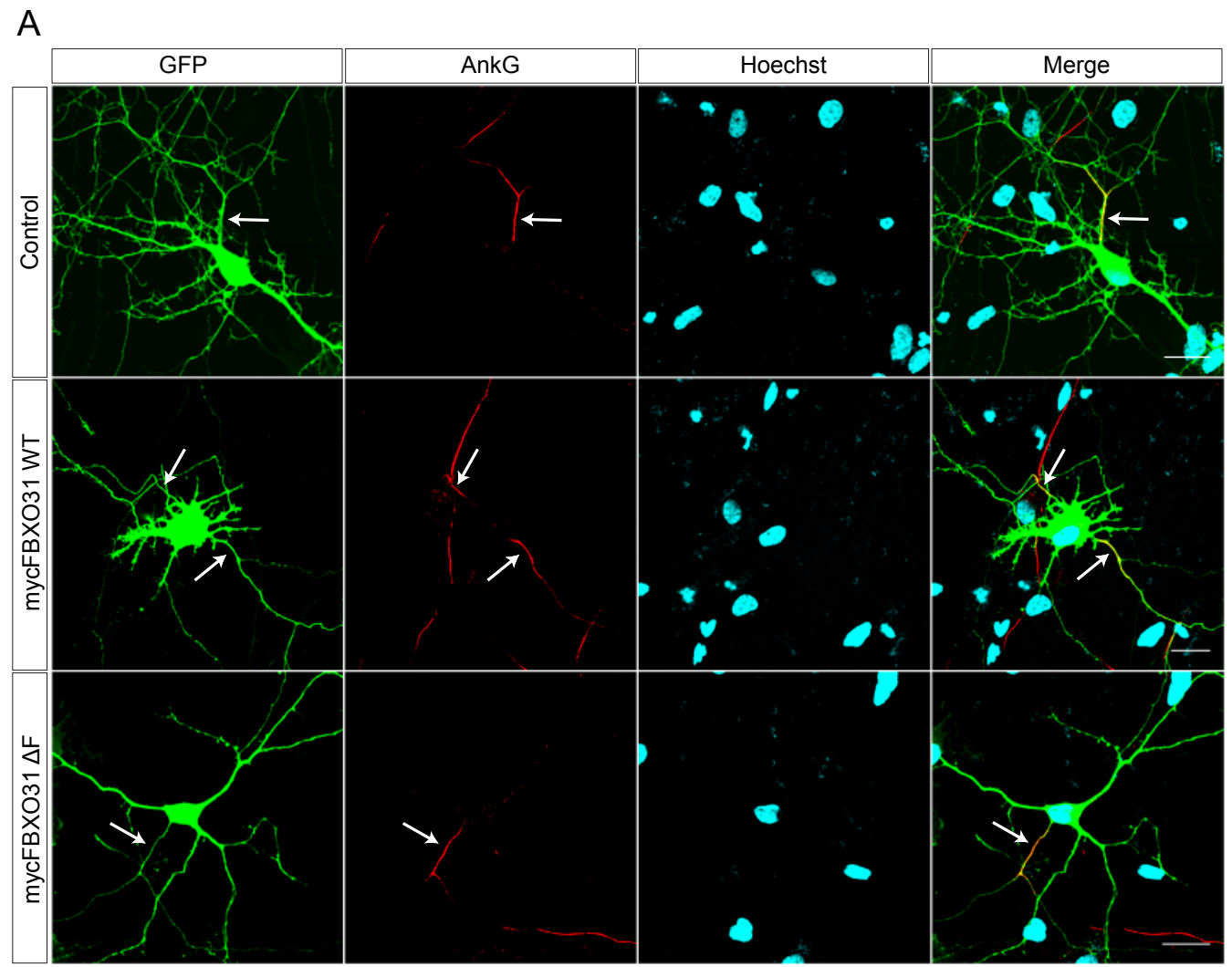

B

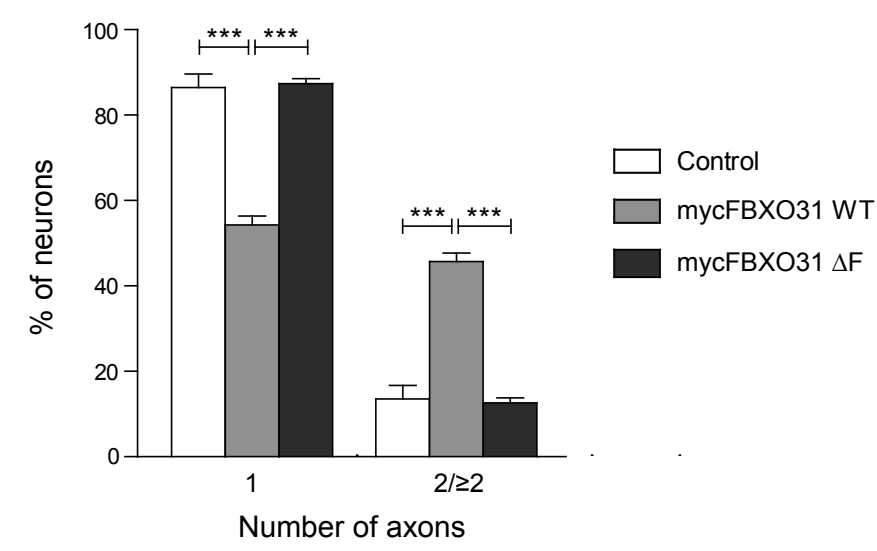

Figure 3.18: FBXO31 gain-of-function promotes multiple axon-phenotype in hippocampal neurons. (A) Representative images of cultured hippocampal neurons transfected with control, FBXO31 WT or FBXO31 $\Delta \mathrm{F}$ plasmids together with GFP and Bcl-XL expression plasmids at DIV 1 and analyzed at DIV 7. The neurons were immunostained with anti-GFP and anti-AnkG antibodies. Arrows indicate AIS. Scale bar equals $10 \mu \mathrm{m}$. (B) Quantification of percentage of neurons with one or more axons in hippocampal neurons as shown in (A). A total of 169 neurons were analyzed in 3 independent sets of experiments. Percentage of neurons with one axon - Control: 86.47 \pm 0.92 ; mycFBXO31 $W T: 54.32 \pm 0.58$ and mycFBXO31 $\Delta F: 87.37 \pm 0.34$. Percentage of neurons with $\geq 2$ axons - Control: $13.53 \pm 0.92$, mycFBXO31 WT: $45.68 \pm 0.58$ and mycFBXO31 $\Delta F: 12.63 \pm 0.34$. Values indicate mean \pm SEM (two-way ANOVA, ${ }^{* * *} \mathrm{p}<0.001$ ). 

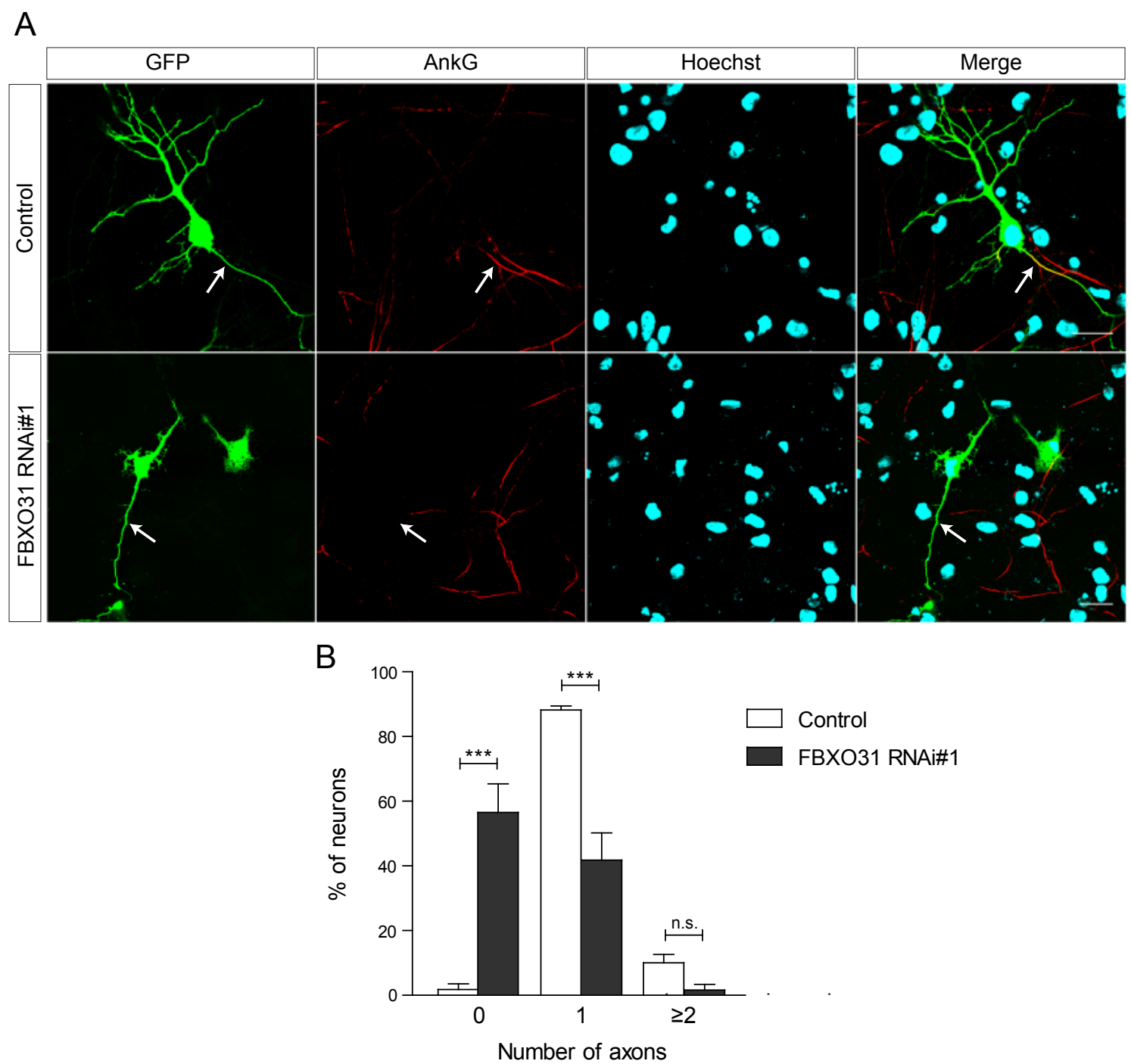

Figure 3.19: FBXO31 knockdown induces no-axon phenotype in hippocampal neurons.

(A) Representative images of cultured hippocampal neurons transfected with control or FBXO31 RNAi\#1/CMV-GFP together with Bcl-X $x_{L}$ at DIV 1 and analyzed at DIV 6. Neurons were immunostained with anti-GFP and anti-AnkG antibodies. Note the loss of AnkG staining at the AIS in FBXO31 RNAi\#1 condition. Arrows indicate AIS. Scale bar equals $10 \mu \mathrm{m}$. (B) Quantification of percentage of neurons with 0,1 or $\geq 2$ axons in hippocampal neurons as shown in (A). A total of 121 neurons were analyzed in 3 independent sets of experiments. Percentage of neurons with no axon - Control: $1.75 \pm 1.75$ and FBXO31 RNAi\#1: $56.51 \pm 8.88$; percentage of neurons with one axon - Control: $88.22 \pm 1.25$ and FBXO31 RNAi\#1: $41.82 \pm 8.41$; percentage of neurons with $\geq 2$ axons - Control: $10.02 \pm 2.61$ and FBXO31 RNAi\#1: 1.67 \pm 1.67 . Values indicate mean \pm SEM (two-way ANOVA, ${ }^{* * *} \mathrm{p}<0.001$, n.s. $=$ not significant). 
knockdown neurons have shorter dendrite lengths as compared to control vector electroporated neurons in the developing cerebellum (Figure 3.20). I was unable to measure the axon lengths as they fasciculate in the molecular layers as untraceable fibers. Besides a reduction in dendrite lengths upon FBXO31 knockdown, I also observed that while most of the control vector electroporated neurons migrate into the IGL, about 50\% of FBXO31 knockdown neurons stall in the ML/EGL and do not migrate into the IGL (Figure 3.21A,B). This suggests a role of FBXO31 in migration of CGNs in developing cerebellum. I also measured the distance of the cell body of individual neurons from the pial surface to estimate their extent of migration. I found that a large proportion of CGNs fails to migrate under FBXO31 knockdown condition as compared to control (Figure 3.21A,C).
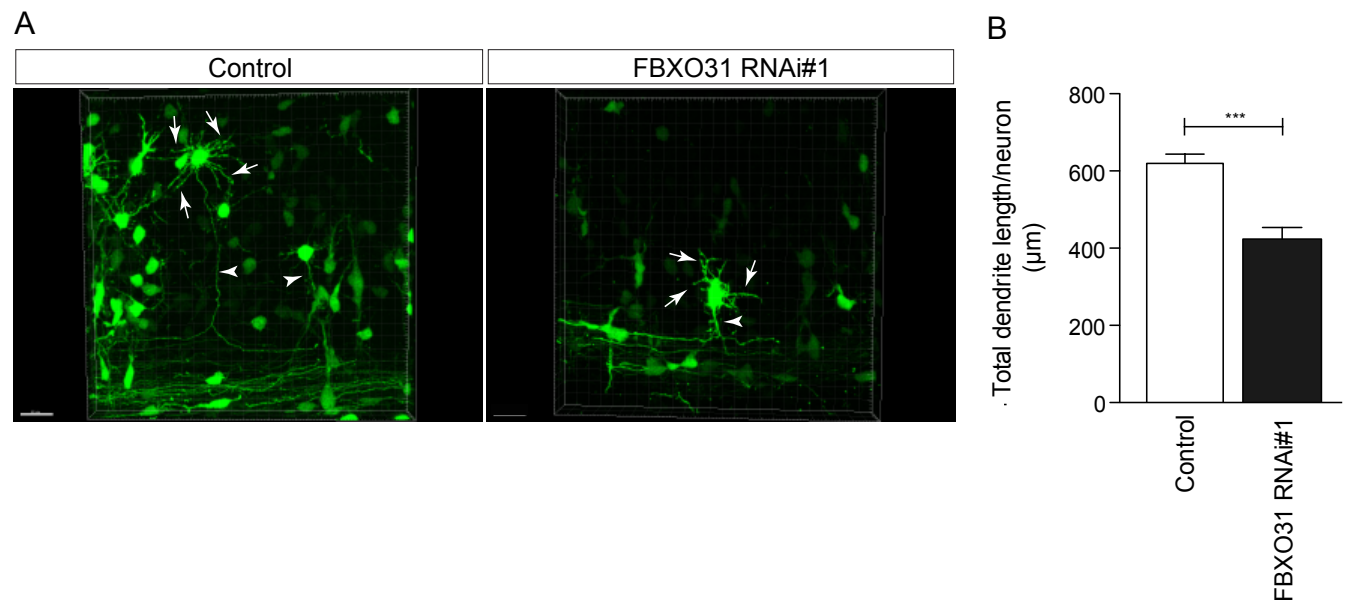

Figure 3.20: FBXO31 knockdown decreases dendrite growth of cerebellar granule neurons in vivo. (A) Representative confocal images of cerebellar granule neurons from rat pups electroporated with control or FBXO31 RNAi\#1/CMV-GFP plasmids together with $\mathrm{Bcl}-\mathrm{X}_{\mathrm{L}}$ at $\mathrm{P} 4$ and analyzed at P9. Total dendrite lengths were analyzed in 3D using Imaris software. Arrows indicate dendrites and arrowheads indicate axons of granule neurons. Scale bar equals $50 \mu \mathrm{m}$. (B) Quantification of total dendrite lengths of granule neurons as shown in (A). A total of 84 neurons were analyzed from three pups each for control and FBXO31 RNAi\#1. Control: $619.8 \pm 23.8 \mu \mathrm{m}$ and FBXO31 RNAi\#1: $423.4 \pm 30.03 \mu \mathrm{m}$. Values indicate mean \pm SEM (unpaired $t$-test, ${ }^{* \star *} \mathrm{p}<0.001$ ). The in vivo electroporations were done by Dr. Judith Stegmüller, while I assisted her with the electroporation and further processed the cerebella. 

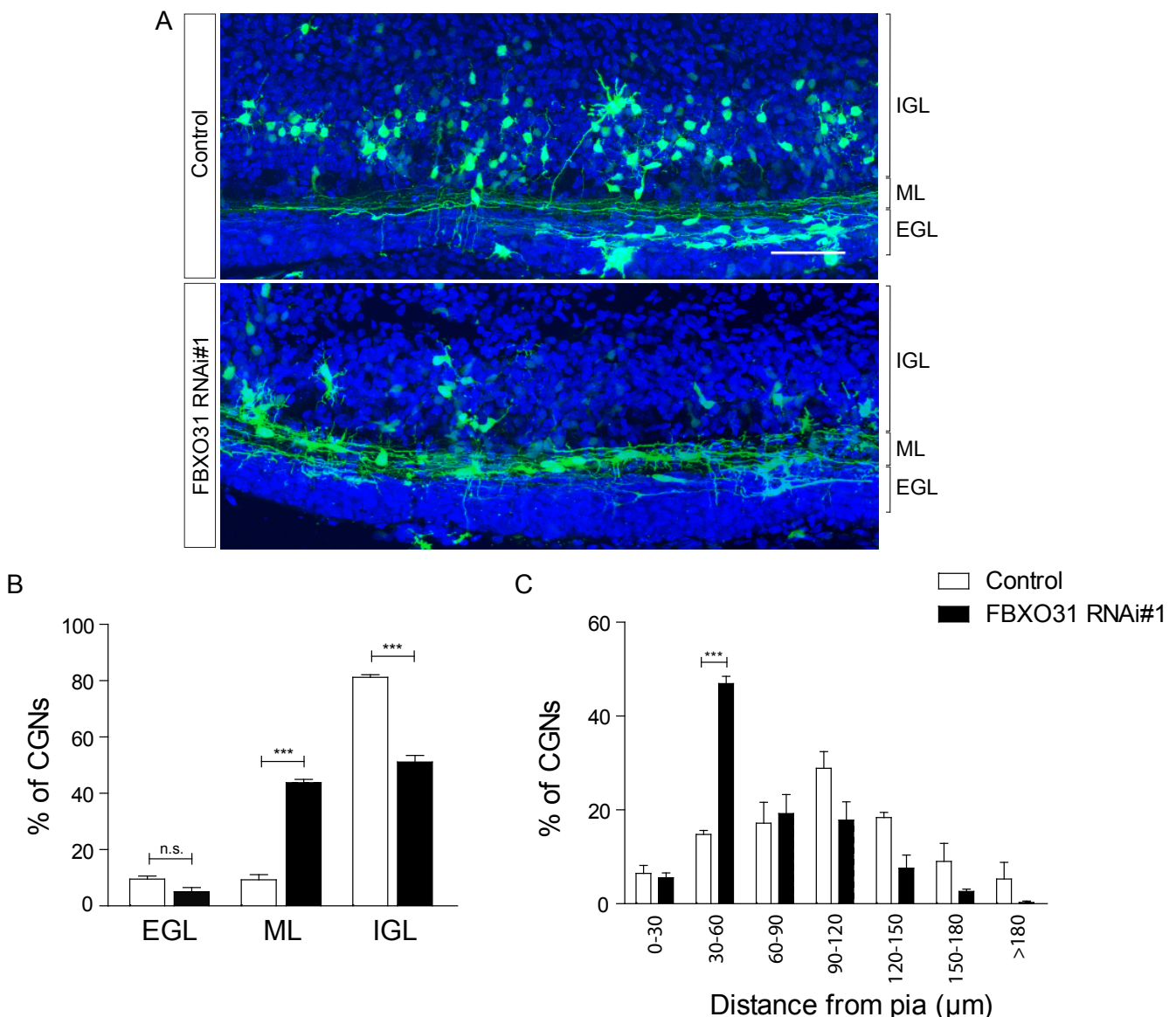

Figure 3.21: FBXO31 promotes neuronal migration of cerebellar granule neurons in developing cerebellum in vivo. (A) Representative confocal images of coronal sections of cerebella from rat pups electroporated with control or FBXO31 RNAi\#1/CMV-GFP plasmids together with $\mathrm{Bcl}-\mathrm{x} \mathrm{L}$ at $\mathrm{P} 4$ and analyzed at $\mathrm{P} 9 . \mathrm{IGL}=$ internal granule layer, $\mathrm{ML}=$ molecular layer, $\mathrm{EGL}=$ external granule layer. Scale bar equals $50 \mu \mathrm{m}$. (B) Quantification of percentage of granule neurons in different layers of cerebellum as shown in (A). A total of 3637 neurons were counted from three pups each for control and FBXO31 RNAi\#1. Percentage of cells in different layers: $E G L$ - Control: 9.52 \pm 1.13 and FBXO31 RNAi\#1: 5.07 $\pm 1.44 ;$ ML - Control: $9.29 \pm 1.81$ and FBXO31 RNAi\#1: 43.82 \pm 1.19 ; IGL - Control: $81.83 \pm 0.97$ and FBXO31 RNAi\#1: 51.11 \pm 2.36 . Values indicate mean \pm SEM (two-way ANOVA, ${ }^{* * *} \mathrm{p}<0.001$, n.s. $=$ not significant). (C) Quantification of distance migrated from pial surface for granule neurons as shown in (A). A total of 681 neurons were analyzed from three pups each for control and FBXO31 RNAi\#1. Percentage of granule neurons: Control - 0-30: 6.49 \pm 1.68 ; 30-60: 14.76 $\pm 0.84 ; 60-90: 17.16 \pm 4.47 ; 90-120: 28.88 \pm 3.53 ; 120$ 150: 18.39 \pm 1.1 ; 150-180: 9.02 \pm 3.83 ; > 180: 5.28 \pm 3.55 ; FBXO31 RNAi\#1 - 0-30: 5.53 \pm 1.04 ; 30-60: 46.93 $\pm 1.54 ; 60-90: 19.22 \pm 4.07 ; 90-120$ : $17.8 \pm 3.89 ; 120-150: 7.59 \pm 2.8 ; 150-180$ : $2.62 \pm 0.51 ;>180: 0.29 \pm 0.29$. Values indicate mean \pm SEM (two-way ANOVA, ${ }^{* * *} p<0.001$ ). The in vivo electroporations were done by Dr. Judith Stegmüller while I assisted her with the electroporation and further processed the samples. 


\subsection{Par6c is a substrate of FBXO31-SCF in con- trol of axon growth}

In the previous section, I observed an important role of FBXO31 during the development of the neurons both in vitro and in vivo. In order to delineate the mechanism underlying the phenotypes observed with FBXO31, I screened for candidates, as interaction partners and putative substrates, localized at the centrosome owing to the centrosomal localization of FBXO31.

\subsubsection{FBXO31 interacts with Par/aPKC complex}

The par polarity complex consists of Par6 protein (Par6 $\alpha$, Par6 $\beta$ and Par6 $\gamma$ ), atypical protein kinase $\mathrm{C}(\mathrm{PKC} \iota$ and $\mathrm{PKC} \zeta)$ and Par3 (Assémat et al., 2008). This is a key complex crucial to progenitor proliferation, neuronal polarization, axon extension, neuronal migration and synapse formation (Solecki et al., 2004; Zhang and Macara, 2006, 2008; Costa et al., 2008; Famulski et al., 2010; Yi et al., 2010; Cheng et al., 2011). The Par6/aPKC complex is primarily localized at the centrosome (Solecki et al., 2004; Kodani et al., 2010). It acts together with Cdc42 and Rac1 to regulate neuronal polarization, axon extension and migration. Given that FBXO31 is also localized at the centrosome, I asked if members of the Par6/aPKC complex interact with FBXO31.

\section{FBXO31 interacts with Par6 $\alpha$ and Par6 $\beta$}

To study the interaction of FBXO31 with Par6, I performed co-immunoprecipitation assay. HEK 293T cells were transfected with mycPar6c and Flag-FBXO31 expression plasmids or respective control plasmids. The cells were lysed two days after transfection, immunoprecipitated with anti-Flag antibody and immunoblotted with anti-myc antibody. I found that FBXO31 associates with Par6c (Figure $3.22 \mathrm{~A}$ ). To confirm the specificity of this interaction, I performed a reciprocal coimmunoprecipitation where I immunoprecipitated with anti-myc antibody and im- 
munoblotted with anti-Flag antibody. With the reciprocal co-immunoprecipitation, I was able to detect specific interaction of Par6c with FBXO31 (Figure 3.22B).
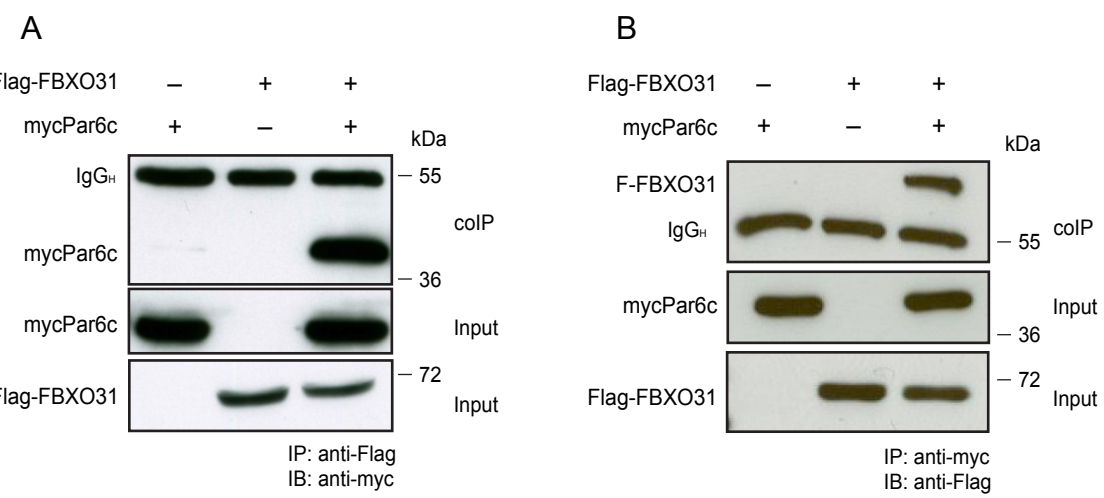

Figure 3.22: FBXO31 interacts with Par6c. (A) Cell lysates of HEK 293T cells, transfected with Flag-FBXO31 and mycPar6c expression plasmids or respective control vectors, were subjected to immunoprecipitation with anti-myc antibody and immunoblotted for Par6c. Inputs represent $5 \%$ of total cell lysates prior to immunoprecipitation. (B) Cell lysates of HEK 293T cells, transfected with Flag-FBXO31 and mycPar6c expression plaids or respective control vectors, were subjected to immunoprecipitation with anti-myc antibody and immunoblotted for FBXO31. Inputs represent $5 \%$ of total cell lysate prior to immunoprecipitation. $\lg G_{H}=$ immunoglobulin heavy chain.

To further characterize this interaction, my colleague Nicola SchwedhelmDomeyer and I generated various myc-tagged deletion mutants of Par6c (Figure 3.23B) and performed co-immunoprecipitation assay of these deletion mutants with GFP-FBXO31. HEK 293T cells were transfected with GFP-FBXO31 or control plasmid together with mycPar6c WT or Par6c deletion mutants. The cells were lysed after two days and the lysate was subjected to immunoprecipitation with antimyc antibody and immunoblotted with anti-GFP antibody. I found that FBXO31 interacts with Par6c-(161-346) and with Par6c-PDZ domain but not with Par6c-(1160) and Par6c-(251-346) (Figure 3.23A,B). Therefore, I concluded that FBXO31 interacts with the PDZ domain of Par6c. 
A

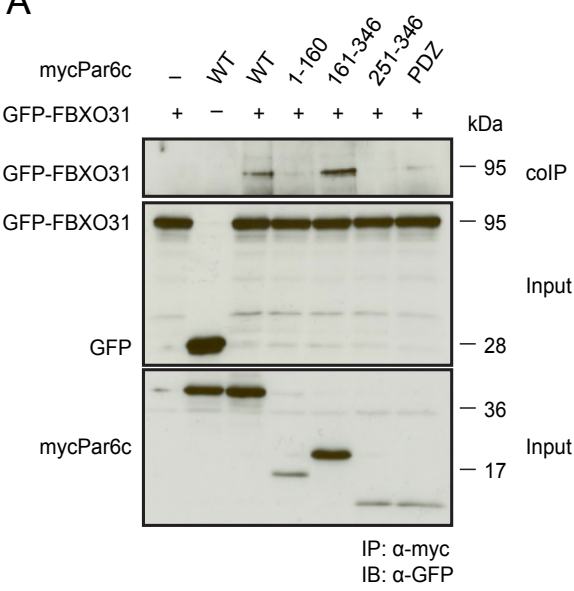

B

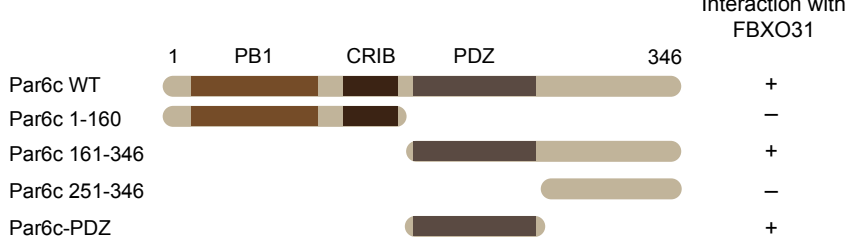

Figure 3.23: Par6c-PDZ domain interacts with FBX031. (A) Lysates of HEK 293T cells, transfected with GFP-FBXO31 together with mycPar6c WT or various deletion mutants, were subjected to immunoprecipitation with anti-myc antibody and probed for FBXO31. Inputs represent $30 \mu \mathrm{g}$ of total protein lysate prior to immunoprecipitation. (B) Schematic of interaction of various Par6c deletion mutants with FBXO31. Par6c deletion mutants were generated by Nicola Schwedhelm-Domeyer and me.

Since Par6c and Par6b share a high sequence homology in their PDZ domain (Figure 3.24), I asked if Par6b also interacts with FBXO31. I transfected HEK 293T cells with mycPar6b and GFP-FBXO31 expression plasmids and respective control plasmids for co-immunoprecipitation assay. Cells were lysed and the lysates

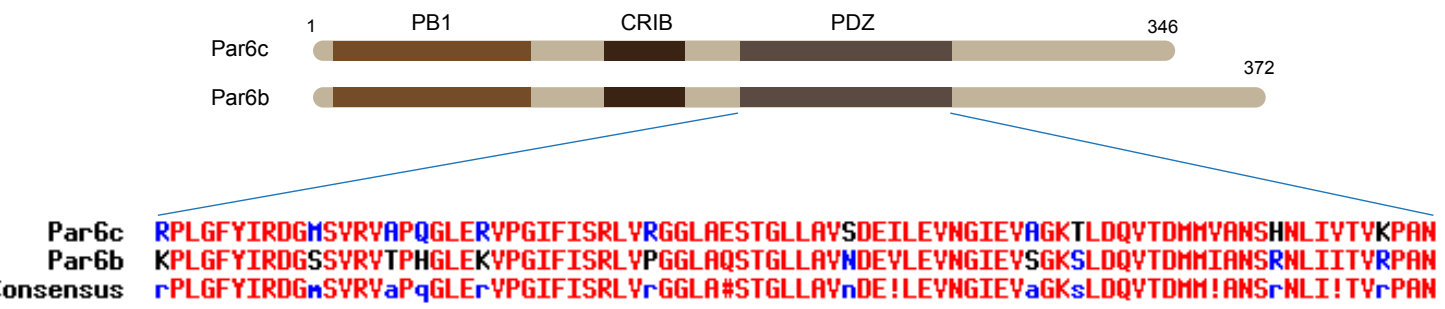

Figure 3.24: Par6c and Par6b share a high sequence similarity in their PDZ domains. Schematic showing sequence homology between PDZ domains of Par6c and Par6b. PB1, CRIB and PDZ domains are highly conserved between Par6c and Par6b. 
were subjected to immunoprecipitation with anti-myc antibody and immunoblotted with anti-GFP antibody. I found that FBXO31 also interacts with Par6b (Figure $3.25)$.

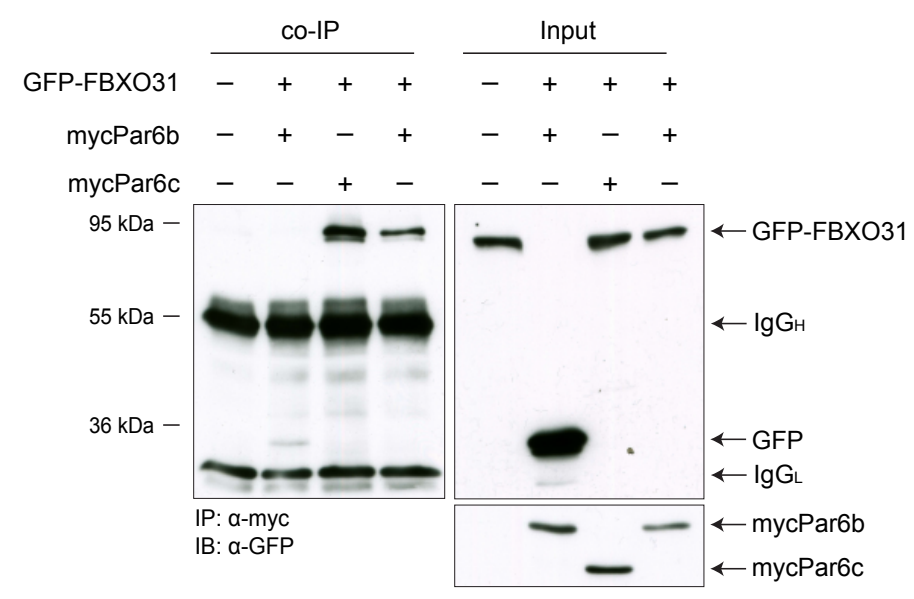

Figure 3.25: FBXO31 interacts with Par6b. Cell lysates of HEK 293T cells, transfected with GFP-FBXO31 and mycPar6b or mycPar6c expression plasmids together with respective control plasmids, were subjected to immunoprecipitation with anti-myc antibody and immunoblotted for FBXO31. Inputs represent $30 \mu \mathrm{g}$ of total protein lysate prior to immunoprecipitation.

\section{FBXO31 interacts with aPKC}

Besides Par6, I also examined if aPKC (PKC $\iota$ and $\mathrm{PKC} \zeta)$ interacts with FBXO31. I transfected the HEK 293T cells with mycFBXO31 and wild-type and dominant negative (DN) form of $\mathrm{HA}-\mathrm{PKC} \iota$ or $\mathrm{HA}-\mathrm{PKC} \zeta$ and respective control vectors. Upon immunoprecipitation with anti-HA antibody and immunoblotting with anti-myc antibody, I found FBXO31 to interact with both the WT and DN form of $\mathrm{PKC} \iota$ (Figure 3.26A) and $\mathrm{PKC} \zeta$ (Figure 3.26B).

\section{FBXO31 does not interact with Par3b}

I also tested the interaction of FBXO31 with another member of Par/aPKC complex - Par3b, a protein phosphorylated by aPKC. I transfected the HEK 293T cells with Flag-FBXO31 and mycPar3b and respective controls, immunoprecipitated the lysates at DIV 2 with anti-Flag antibody and immunoblotted with anti-myc 
A

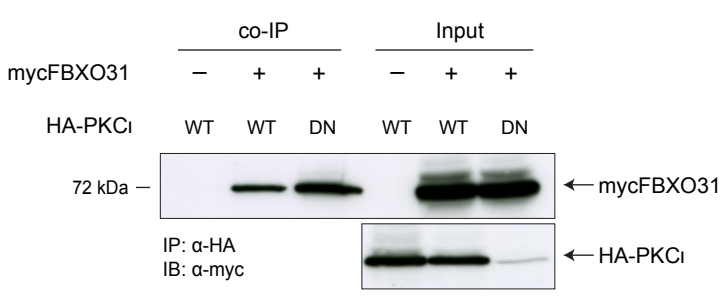

B

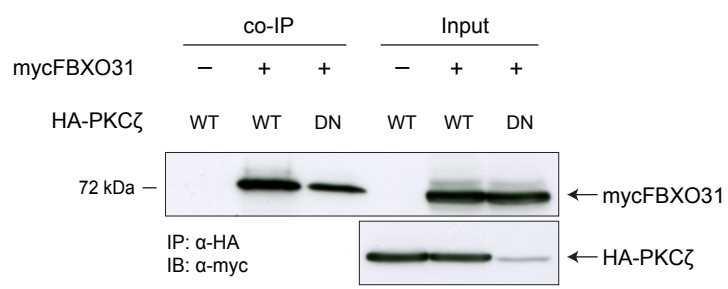

Figure 3.26: FBXO31 interacts with $\operatorname{PKC}_{\iota}$ and $\operatorname{PKC} \zeta$. (A) Lysates of HEK 293T cells, transfected with mycFBXO31 and $\mathrm{HA}-\mathrm{PKC} \iota \mathrm{WT}$ or $\mathrm{DN}$ and respective control vectors were subjected to immunoprecipitation with anti-HA antibody and immunoblotted for FBXO31. Inputs represent $5 \%$ of total protein lysate prior to immunoprecipitation. (B) Lysates of HEK 293T cells transfected with mycFBXO31 and $\mathrm{HA}-\mathrm{PKC} \zeta \mathrm{WT}$ or DN and respective control vectors were subjected to immunoprecipitation with anti-HA antibody and immunoblotted for FBXO31. Inputs represent $5 \%$ of total protein lysate prior to immunoprecipitation.

antibody. I did not find any interaction of FBXO31 with Par3b (Figure 3.27), suggesting that FBXO31 interacts only with the core members of Par/aPKC complex, namely Par6 and aPKC.

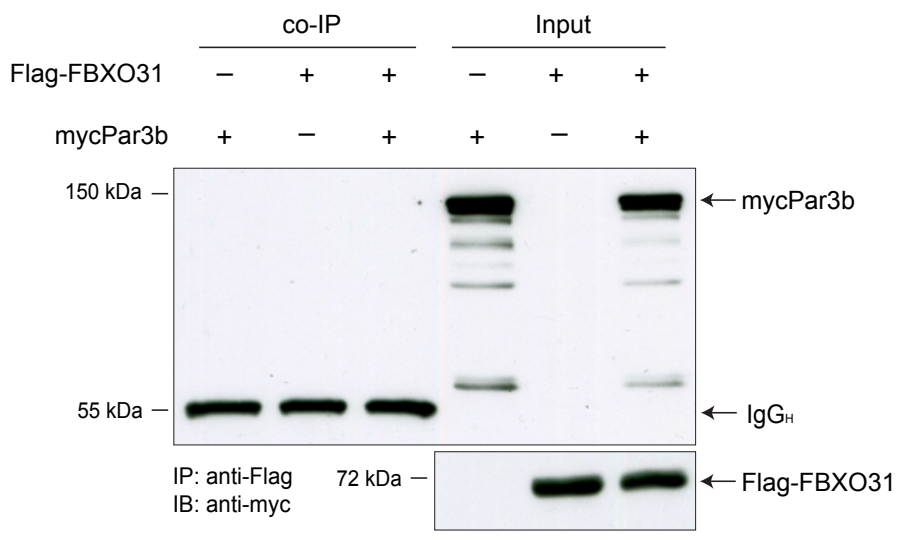

Figure 3.27: FBXO31 does not interact with Par3b. Lysates of HEK $293 \mathrm{~T}$ cells, transfected with Flag-FBXO31 and mycPar3b plasmids and respective control vectors, were subjected to immunoprecipitation with anti-Flag antibody and immunoblotted for Par3b. Inputs represent $5 \%$ of

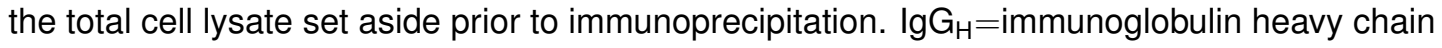




\subsubsection{Par6c but not $\mathrm{PKC} \zeta$ is degraded by the ubiquitin proteasome system in granule neurons}

Next, I tested whether Par6c and aPKC are degraded in a proteasome-dependent manner in the cultured cerebellar granule neurons. I transfected the neurons with mycPar6c expression plasmid at DIV 0 and treated the cells with a proteasome inhibitor lactacystin or vehicle, $10 \mathrm{hr}$ prior to lysis at DIV3. The lysates were immunoblotted with anti-myc antibody. I found a significant increase in levels of mycPar6c upon inhibition of the proteasome with lactacystin suggesting that Par6c is degraded in a proteasome-dependent manner in the CGNs (Figure 3.28A).

I also analyzed whether $\mathrm{PKC} \zeta$ is degraded in a proteasome-dependent manner. For this, I treated the cultured cerebellar granule neurons at DIV 2 with 10 $\mu \mathrm{M}$ lactacystin or vehicle DMSO for $10 \mathrm{hrs}$ and probed the lysates with anti-PKC $\zeta$ antibody. Unlike Par6c, I did not find any change in the levels of $\mathrm{PKC} \zeta$ upon treatment with lactacystin suggesting that while Par6c undergoes proteasome-dependent degradation in CGNs, $\mathrm{PKC} \zeta$ does not (Figure 3.28B).
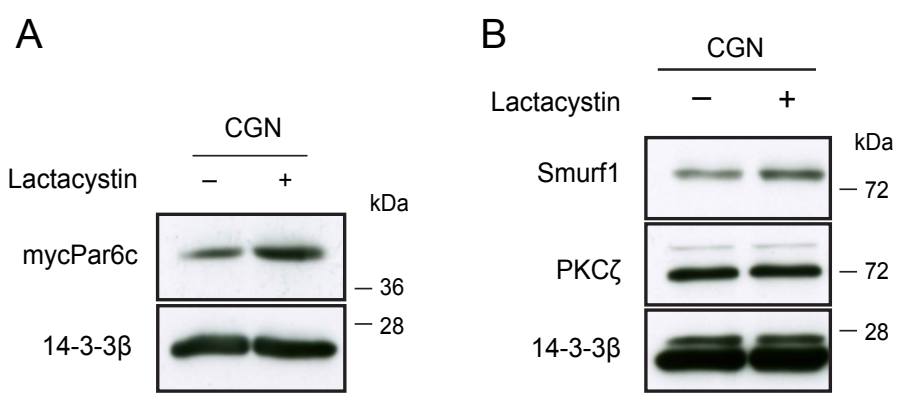

Figure 3.28: Par6c but not $\operatorname{PKC} \zeta$ is degraded in a proteasome-dependent manner in cerebellar granule neurons. (A) Cultured cerebellar granule neurons were transfected with mycPar6c together with GFP and Bcl- $\mathrm{X}_{\mathrm{L}}$ plasmids at DIV 0 and were treated with vehicle or $10 \mu \mathrm{M}$ lactacystin for $10 \mathrm{hrs}$ prior to lysis at DIV 3. Neuronal lysates were immunoblotted for Par6c. 14-3-3 $\beta$ was used as a loading control. (B) Cultured cerebellar granule neurons were treated with vehicle or $10 \mu \mathrm{M}$ lactacystin for $10 \mathrm{hrs}$ prior to lysis at DIV 3. Cell lysates were immunoblotted for PKC $\zeta$. Smurf1 and 14-3-3 $\beta$ served as positive control and loading control, respectively. 


\subsubsection{FBXO31 regulates the stability of Par6c}

Since I observed Par6c to be degraded in a proteasome-dependent manner, I asked whether FBXO31 regulates Par6c stability. To test this, I transfected HEK 293 T cells with mycPar6c plasmid together with Flag-FBXO31 expression plasmid or empty vector control. I also transfected HEK 293T cells with mycPar6c together with FBXO31 RNAi plasmid or with U6 empty vector control. The cells were lysed at DIV 2 and DIV 4 respectively and probed with anti-myc antibody to detect the levels of Par6c. I observed that with FBXO31 overexpression, Par6c levels were downregulated (Figure 3.29A), whereas with FBXO31 knockdown, Par6c levels were significantly upregulated (Figure 3.29B). These experiments suggest that Par6c is regulated by the E3 ubiquitin ligase FBXO31-SCF.

A

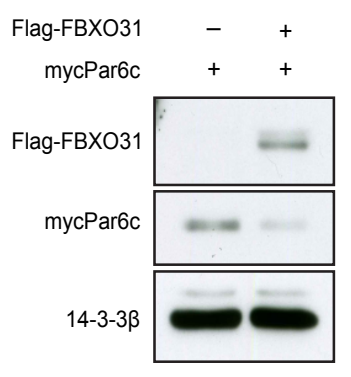

B

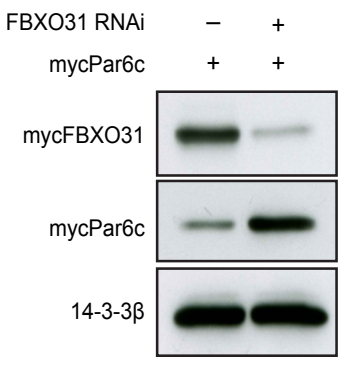

C

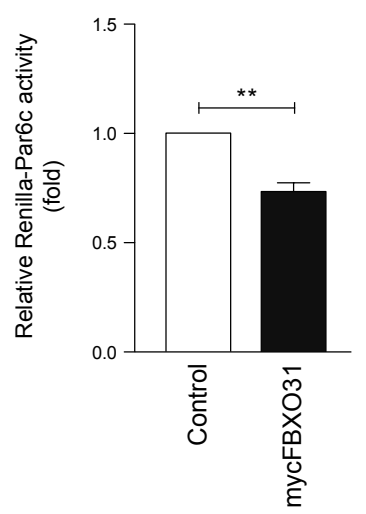

D

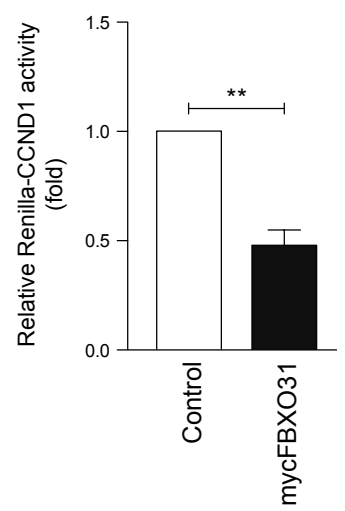

Figure 3.29: FBXO31 regulates the stability of Par6c. (A) HEK 293T cells were transfected with mycPar6c expression plasmid together with control or Flag-FBXO31. Two days after transfection the cell lysates were immunoblotted for FBXO31 and Par6c. 14-3-3 $\beta$ was used as a loading control. (B) HEK 293T cells were transfected with mycPar6c and mycFBXO31 expression plasmids together with control or FBXO31 RNAi\#1. The cells were lysed four days after transfection and the cell lysates were immunoblotted for FBXO31 and Par6c. 14-3-3 $\beta$ was used as a loading control. (C-D) HEK 293T cells were transfected with Renilla-Par6c (C) and Renilla-CCND1 (D) together with control or mycFbxo31 WT and SV40 firefly luciferase. The cell lysates were assessed for Renilla activity using dual-luciferase assay. Histogram indicates mean+SEM (unpaired $t$-test, $\left.{ }^{* *} \mathrm{p}<0.01\right)$ 
In addition, I performed dual-luciferase assay as another approach to determine the stability of Par6c upon overexpression of FBXO31. HEK 293T cells transfected with Renilla-Par6c expression plasmid together with mycFBXO31 or empty vector control and internal control SV40 firefly luciferase were subjected to dual luciferase assay. The firefly and renilla luciferase substrates and the luminometer were kindly provided by Dr. Moritz Rossner (Max Planck Institute of Experimental Medicine, Goettingen, Germany). While I observed 30\% reduction in activity of Renilla-Par6c (Figure 3.29C) upon FBXO31 overexpression as compared to control, I also observed a 52\% decrease in Renilla-Cyclin D1 activity in presence of FBXO31 (Figure 3.29D). Cyclin D1 is targeted to proteasome for degradation by FBXO31-SCF (Santra et al., 2009). Taken together, I found that FBXO31 regulates Par6c levels.

\subsubsection{Par6c is polyubiquitinated and targeted for proteasomal degra- dation by FBXO31}

To examine whether FBXO31-SCF is responsible for Par6c ubiquitination, my colleague Nicola Schwedhelm-Domeyer and I carried out cell-based ubiquitination assay. We expressed Par6c together with control vector, FBXO31 WT or FBXO31 $\Delta \mathrm{F}$. The lysates were subjected to immunoprecipitation for Par6c and immunoblotted with ubiquitin antibody. While we found a sparse ubiquitination of Par6c in control and FBXO31 $\Delta \mathrm{F}$ conditions, wild-type FBXO31 potently stimulates polyubiquitination of Par6c (Figure 3.30).

To confirm that the polyubiquitination of Par6c contributes to its proteasomal turnover, we examined the linkage of the polyubiquitination chain associated with Par6c. Ubiquitin chains can be assembled via different lysines in ubiquitin. Ubiquitin chains that are linked via lysine 48 (K48) are well known to trigger the degradation of proteins, while K63-linkage of ubiquitin represents a non-proteolytic modification. In further cell-based ubiquitination assays, we found that FBXO31 triggered the assembly of a K48-linked but not K63-linked polyubiquitin chain of 


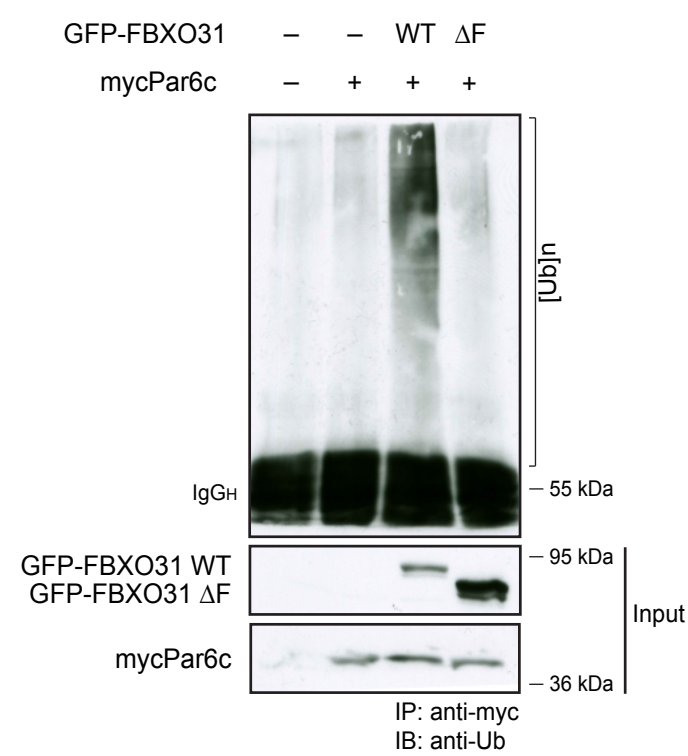

Figure 3.30: Par6c is polyubiquitinated by FBX031-SCF. Lysates of HEK 293T cells, transfected with GFP-Fbxo31 WT or $\Delta \mathrm{F}$ together with mycPar6c plasmid and respective control vectors, were subjected to immunoprecipitation with anti-myc antibody and immunoblotted with the ubiquitin antibody. Nicola Schwedhelm-Domeyer and I performed the cell-based ubiquitination assays.

Par6c (Figure 3.31A \& B), suggesting a degradation-inducing modification of Par6c by the E3 ubiquitin ligase FBXO31-SCF. Collectively, these data indicate that Par6c is targeted for proteasomal degradation by FBXO31-SCF.

\subsubsection{Par6c acts as a suppressor of axon growth but not dendrite growth}

Par6/aPKC complex is a key regulator of neuronal polarity (Shi et al., 2003; Nishimura et al., 2005; Schwamborn et al., 2007; Yi et al., 2010; Cheng et al., 2011). I asked if Par6c, in addition to its role in polarity, has axon or dendrite growthregulating functions. 
A

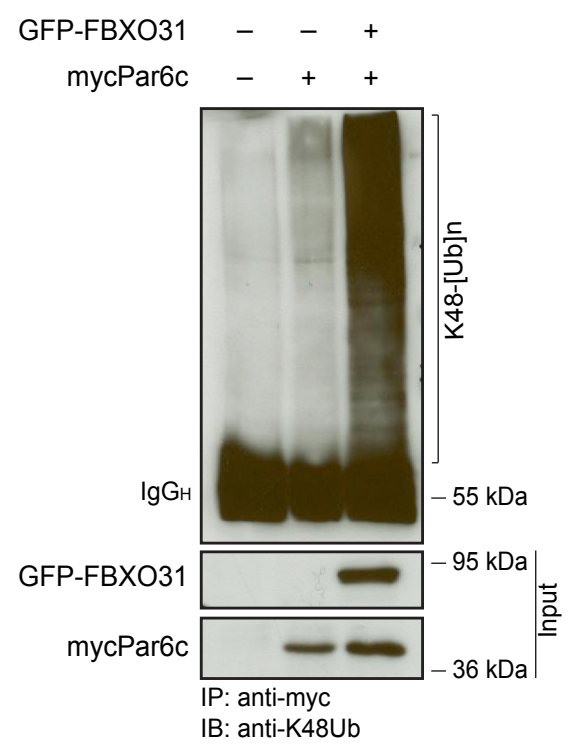

B

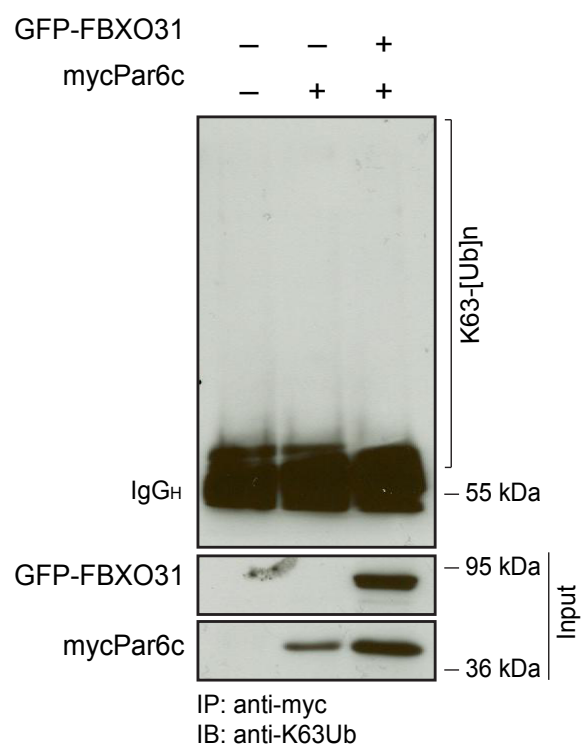

Figure 3.31: Par6c polyubiquitination by FBXO31-SCF is K48-linked and not K63-linked. (A) Lysates of HEK 293T cells, transfected with GFP-FBXO31 and mycPar6c plasmids and respective control vectors, were subjected to immunoprecipitation with anti-myc antibody and immunoblotted with K48-specific anti-ubiquitin antibody. (B) Lysates of HEK 293T cells, transfected with GFP-FBXO31 and mycPar6c expression plasmids and respective control vectors, were subjected to immunoprecipitation with anti-myc antibody and probed with K63-specific anti-ubiquitin antibody. Nicola Schwedhelm-Domeyer and I performed the cell-based ubiquitination assays.

\subsubsection{Par6c gain-of-function suppresses axon growth but not dendrite growth in neurons}

Since FBXO31 regulates Par6c levels, I reasoned that overexpression of Par6c might phenocopy the FBXO31 RNAi phenotype. In order to test this, I transfected granule neurons with control or Par6c expression plasmid at DIV 0 and analyzed the neurons for axon and dendrite growth three days later. Indeed, I found that Par6c overexpression resulted in shorter axons whereas dendrite growth was not affected (Figure 3.32A-D). I also observed that a large population neurons were not polarized upon Par6c overexpression (Figure 3.32E), which is consistent with the previous role of Par6c in neuronal polarity (Shi et al., 2003; Cheng et al., 2011). 

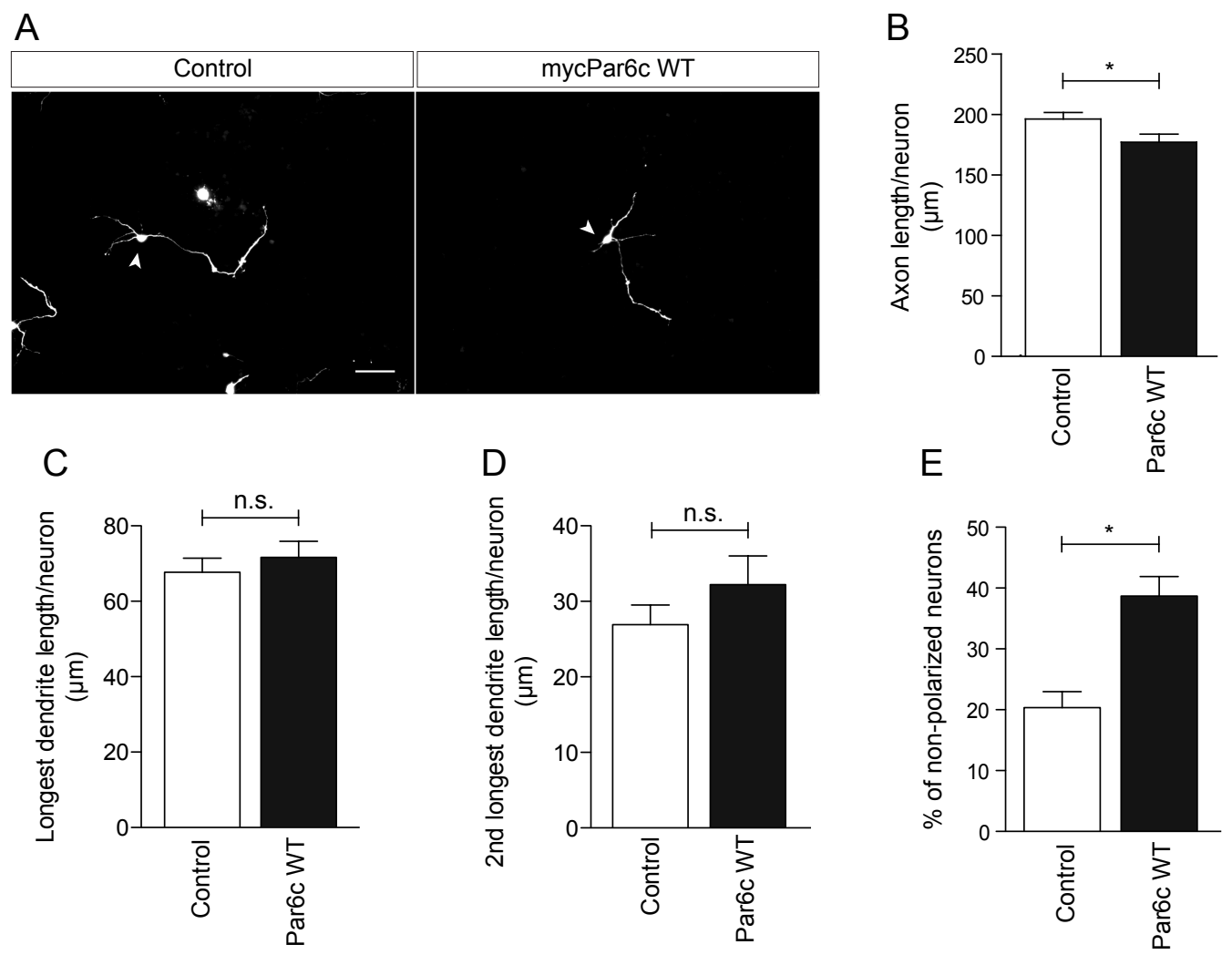

Figure 3.32: Par6c acts as an axon growth suppressor. (A) Representative images of cerebellar granule neurons transfected with control or mycPar6c WT plasmids together with GFP and $\mathrm{Bcl}-\mathrm{X}_{\mathrm{L}}$ plasmids at DIV 0 and analyzed at DIV 3. Arrowheads indicate cell bodies. Scale bar equals $50 \mu \mathrm{m}$. (B) Quantification of axon lengths of granule neurons as shown in (A). A total of 231 neurons were analyzed in 3 independent sets of experiments. Control: $196.3 \pm 5.47 \mu \mathrm{m}$ and mycPar6c WT: $177.3 \pm 6.62 \mu \mathrm{m}$. Values indicate mean \pm SEM (unpaired $t$-test, ${ }^{*} \mathrm{p}<0.05$ ). (C) Quantification of longest dendrite length of granule neurons shown in (A). A total of 160 neurons were analyzed in 3 independent sets of experiments. Control: $67.67 \pm 3.77 \mu \mathrm{m}$ and mycPar6c WT: $71.71 \pm 4.25 \mu \mathrm{m}$. Values indicate mean \pm SEM (unpaired $t$-test, n.s.=not significant). (D) Quantification of second longest dendrite length of granule neurons as shown in (A). A total of 160 neurons were analyzed in 3 independent sets of experiments. Control: $26.91 \pm 2.59 \mu \mathrm{m}$ and mycPar6c WT: $32.22 \pm 3.8 \mu \mathrm{m}$. Values indicate mean \pm SEM (unpaired $t$-test, n.s.=not significant). (E) Quantification of non-polarized neurons for granule neurons shown in (A). A total of 226 neurons were analyzed from 3 independent sets of experiments. Percentage of non-polarized neurons - Control: $20.36 \pm 2.64$ and mycPar6c WT: $38.71 \pm 3.18$. Values indicate mean \pm SEM (unpaired $t$-test, $\left.{ }^{*} \mathrm{p}<0.05\right)$. 


\subsubsection{Par6c loss-of-function promotes axon but not dendrite growth}

\section{Validation of Par6c RNAi construct}

To further investigate the effect of Par6c loss-of-function in neurons, I generated a Par6c RNAi plasmid using the targeting region described before (Zhang and Macara, 2008). To validate the Par6c RNAi construct, HEK 293T cells were transfected with either empty control vector or with Par6c RNAi plasmid together with mycPar6c plasmid. The cell lysates were subjected to immunoblotting for Par6c. I observed a significant reduction in Par6c levels with respect to control (Figure 3.33).

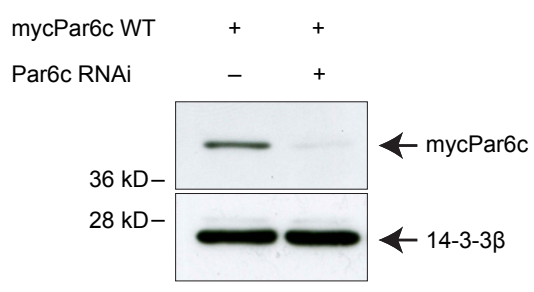

Figure 3.33: Validation of Par6c RNAi. Lysates of HEK 293T cells transfected with mycPar6c expression plasmid together with control or Par6c RNAi plasmid was immunoblotted for Par6c. $14-3-3 \beta$ was used as a loading control.

Par6c knockdown promotes axon growth but does not alter dendrite growth

To address the question of Par6c loss-of-function on axon or dendrite growth, I transfected the granule neurons with control or Par6c RNAi plasmid together with GFP and Bcl- $\mathrm{x}_{\mathrm{L}}$ at DIV 0 and analyzed the cells four days later. I observed a significant increase in axon length but no change in the dendrite length upon Par6c knockdown as compared to control neurons (Figure 3.34A-C). Moreover, I did not find any change in percentage of non-polarized cells upon Par6c knockdown (Figure 3.34D).

To validate the specificity of Par6c RNAi phenotype and rule out the off-target effect of Par6c RNAi, I generated Par6c rescue mutants (Par6c-Res) that are resistant to RNAi-mediated degradation (Figure 3.35A). To validate the Par6c rescue 
A

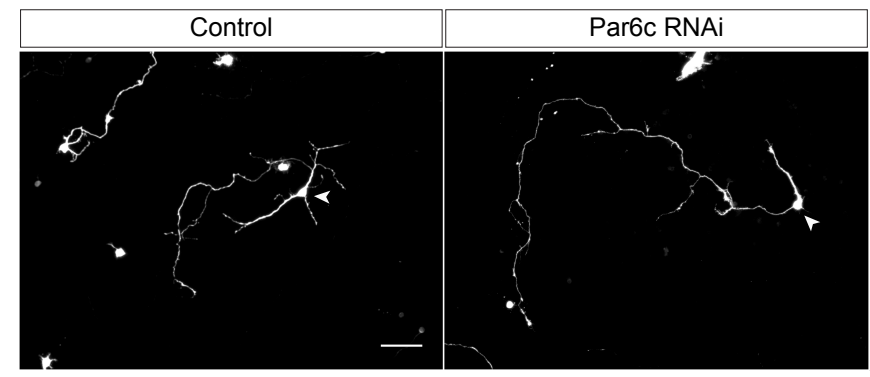

B

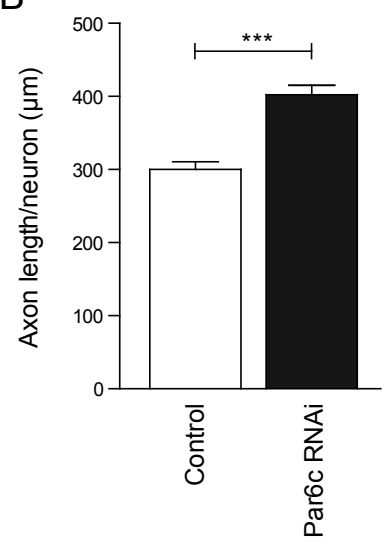

C

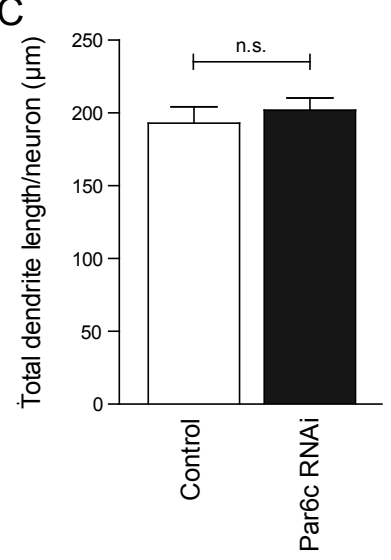

D

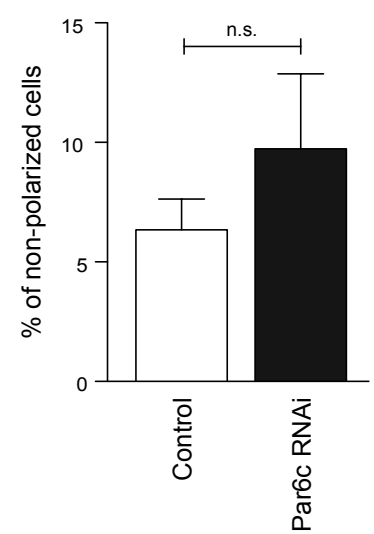

Figure 3.34: Par6c loss-of-function promotes axon but not dendrite growth. (A) Representative images of cerebellar granule neurons transfected with control or Par6c RNAi plasmid together with GFP and BCl-X plasmids at DIV 0 and analyzed at DIV 4. Arrowheads indicate cell bodies. Scale bar equals $50 \mu \mathrm{m}$. (B) Quantification of axon length of granule neurons as shown in (A). A total of 285 neurons were analyzed in 3 independent sets of experiments. Control: $300.3 \pm 10.41 \mu \mathrm{m}$ and Par6c RNAi: $402.5 \pm 12.61 \mu \mathrm{m}$. Values indicate mean \pm SEM (unpaired $t$-test, $\left.{ }^{* * *} \mathrm{p}<0.001\right)$. (C) Quantification of total dendrite lengths of granule neurons as shown in (A). A total of 269 neurons were analyzed from 3 independent sets of experiments. Control: $193.0 \pm 11.28 \mu \mathrm{m}$ and Par6c RNAi: $201.9 \pm 8.44 \mu \mathrm{m}$. Values indicate mean \pm SEM (unpaired $t$-test, n.s.=not significant). (D) Quantification of percentage of non-polarized granule neurons as shown in (A). A total of 291 neurons were analyzed in 3 independent sets of experiments. Percentage of non-polarized neurons - Control: $6.36 \pm 1.28$ and Par6c RNAi: 9.73 \pm 3.13 . Values indicate mean \pm SEM (unpaired $t$-test, n.s. $=$ not significant).

mutants, HEK 293T cells were transfected with Par6c WT or Par6c-Res plasmids together with Par6c RNAi plasmid and respective controls. The cells were lysed four days after transfection and the lysates were subjected to immunoblotting for 
Par6c. Though there was a significant reduction in Par6c levels upon transfection with Par6c RNAi, the levels of Par6c-Res did not respond to Par6c RNAi (Figure 3.35B), thereby confirming that Par6c-Res is resistant to RNAi-mediated degradation.
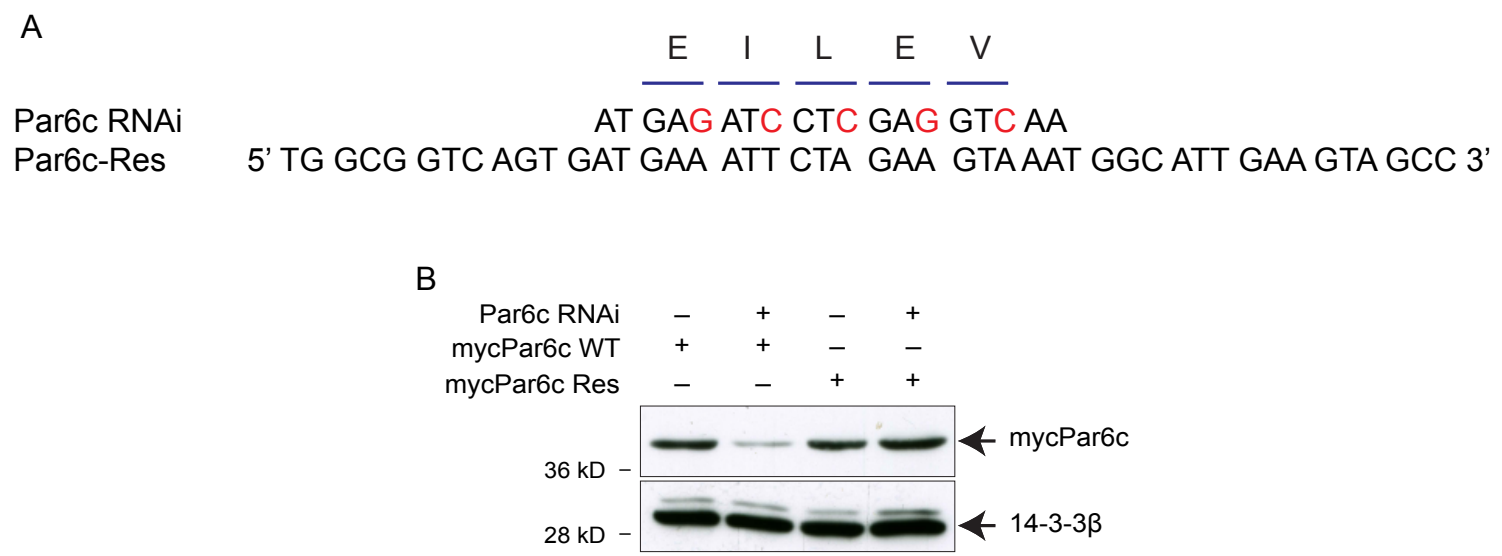

Figure 3.35: Generation and validation of Par6c-Res construct. (A) Silent mutations, as indicated, were introduced into Par6c RNAi target sequence to generate Par6c-Res construct. (B) HEK 293T cells were transfected with control, mycPar6c WT or mycPar6c-Res together with control or Par6c RNAi. Cell lysates were immunoblotted for Par6c. 14-3-3 $\beta$ served as a loading control.

To establish the Par6c RNAi phenotype on axon and dendrite growth, I transfected granule neurons with control, Par6c RNAi or Par6c RNAi together with Par6c-Res plasmid at DIV 0. Neurons were analyzed at DIV 4 for their axon and dendrite lengths. As seen previously, I found that while Par6c knockdown increases axon length, the expression of Par6c-Res reverses this effect (Figure 3.36A-D). These results confirm the specific phenotype of Par6c loss-of-function on axon but not dendrite growth. 


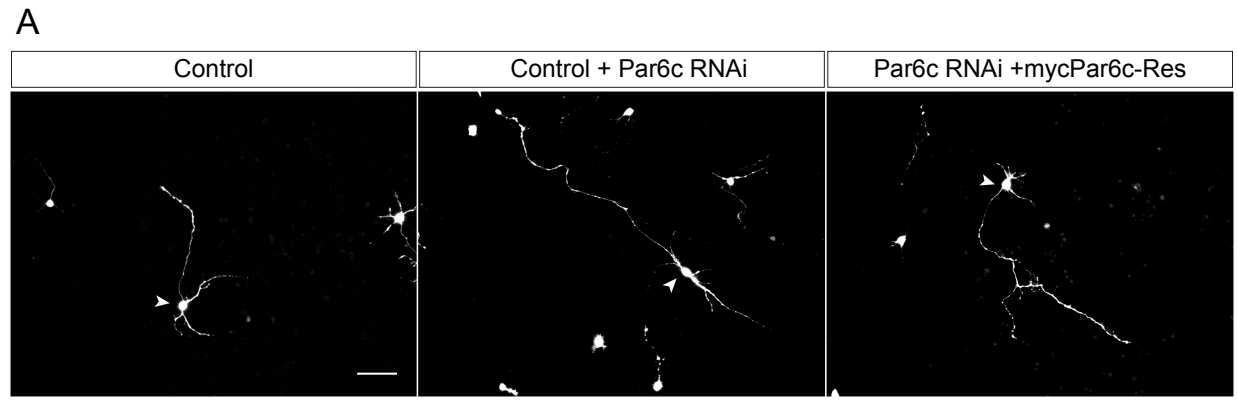

B

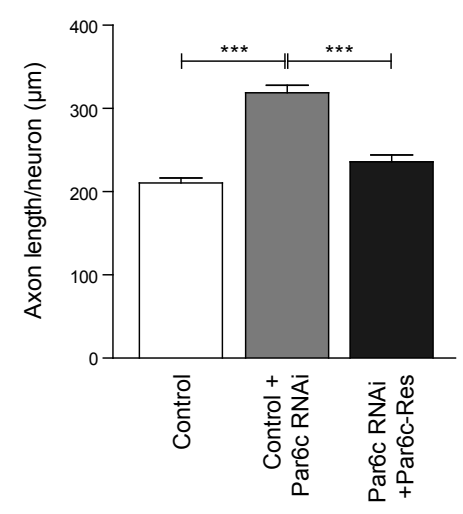

C

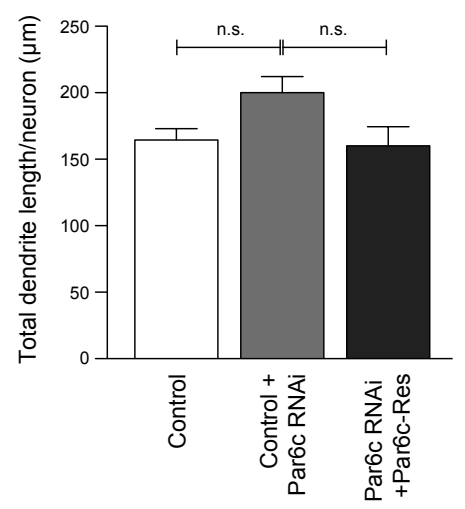

D

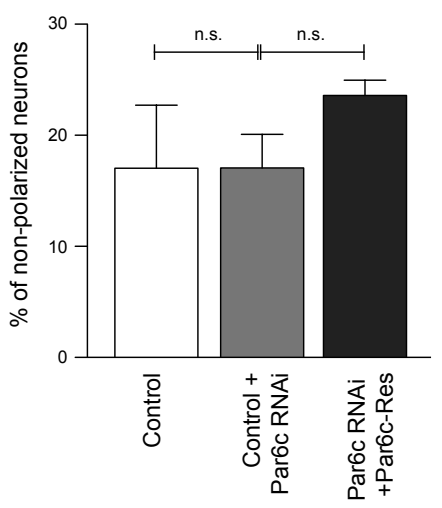

Figure 3.36: Par6c-Res reverses the Par6c knockdown phenotype in axon growth of cerebellar granule neurons. (A) Representative images of cerebellar granule neurons transfected with control vector, Par6c RNAi plasmid or Par6c RNAi plasmid and mycPar6c-Res together with GFP and Bcl- $x_{L}$ expression plasmids at DIV 0 and analyzed at DIV 4. Arrowheads indicate cell bodies. Scale bar equals $50 \mu \mathrm{m}$. (B) Quantification of axon length of granule neurons as shown in (A). A total of 309 neurons were analyzed in 3 independent sets of experiments. Control: 210.6 $\pm 5.83 \mu \mathrm{m}$, Par6c RNAi: $318.7 \pm 8.99 \mu \mathrm{m}$ and Par6c RNAi+mycPar6c-Res: $235.8 \pm 8.23 \mu \mathrm{m}$. Values indicate mean \pm SEM (one-way ANOVA, ${ }^{* * *} \mathrm{p}<0.001$, n.s. $=$ not significant). (C) Quantification of total dendrite lengths of granule neurons as shown in (A). A total of 255 neurons were analyzed in 3 independent sets of experiments. Control: $164.5 \pm 8.57 \mu \mathrm{m}$, Par6c RNAi: 200.0 \pm 12.21 $\mu \mathrm{m}$ and Par6c RNAi+mycPar6c-Res: $160.2 \pm 14.39 \mu \mathrm{m}$. Values indicate mean \pm SEM (one-way ANOVA, n.s.=not significant). (D) Quantification of percentage of non-polarized granule neurons as shown in (A). A total of 313 neurons were analyzed in 3 independent sets of experiments. Percentage of non-polarized neurons - control: 17.05 \pm 5.66 , Par6c RNAi: $17.07 \pm 3.01$ and Par6c $R N A i+m y c P a r 6 c-R e s: 23.59 \pm 1.36$. Values indicate mean \pm SEM (one-way ANOVA, n.s. $=$ not significant). 


\subsubsection{Par6c acts downstream of FBXO31 in control of axon but not dendrite growth}

Since I identified Par6c as a target of FBXO31 and a regulator of axon growth, I determined if Par6c acts as a downstream component of the FBXO31-SCF pathway of neuronal morphogenesis. To establish this, I performed an epistasis analysis and transfected granule neurons with FBXO31 RNAi or Par6c RNAi plasmid or both plasmids at DIV 0 and analyzed their axon and dendrite lengths at DIV 4. As demonstrated previously, I found a reduction in axon and dendrite lengths upon FBXO31 knockdown and an increase in axon length upon Par6c knockdown. Upon FBXO31/Par6c double knockdown, I observed long axons and short dendrites, suggesting that axonal Par6c phenotype has a dominant effect over FBXO31 phenotype, while dendritic FBXO31 phenotype prevails (Figure 3.37A-C). This data suggests that Par6c acts downstream of FBXO31 in control of axon growth but not dendrite growth.

\subsection{FBXO31 interacts with Cdh1-APC in control of axon growth}

Cdh1-APC is a multimeric RING E3 ubiquitin ligase that is composed of several subunits including APC2 that serves as scaffolding protein and Cdh1 or Cdc20, which act as adaptor proteins to recruit the substrates to the complex (Peters, 2006). Cdh1 and Cdc20 recognize substrates harboring destruction box (D-box) motifs (RxxL) or KEN-box motifs (Peters, 1998). While Cdh1/Cdc20-APC has been extensively studied in the context of cell cycle regulation, their role in postmitotic neurons has only recently been characterized. Cdh1-APC has been identified as a suppressor of axon growth by regulating the degradation of transcription factors SnoN and Id2 (Konishi et al., 2004; Lasorella et al., 2006; Stegmüller et al., 2006) 


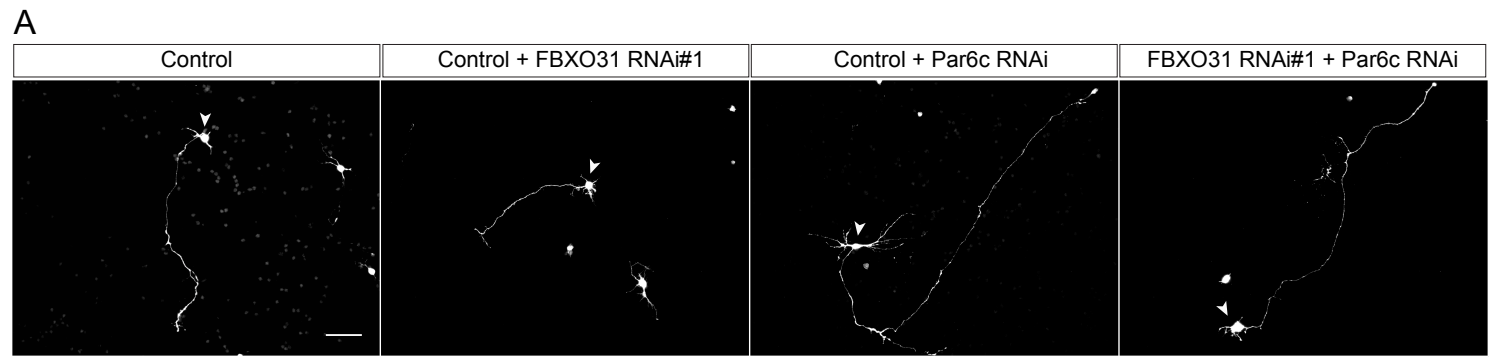

B

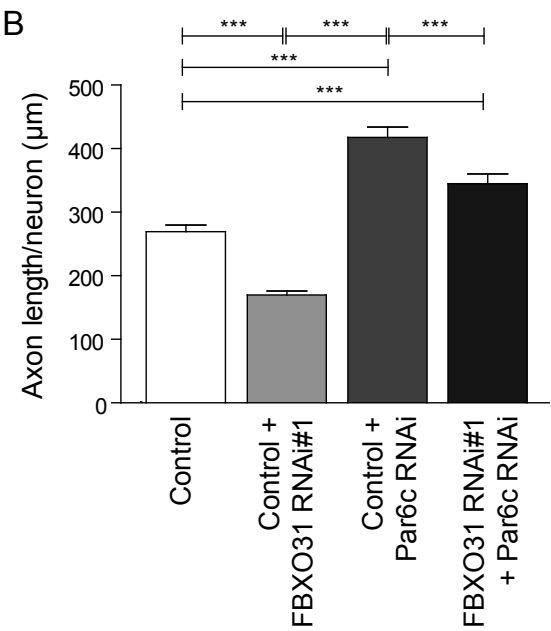

C

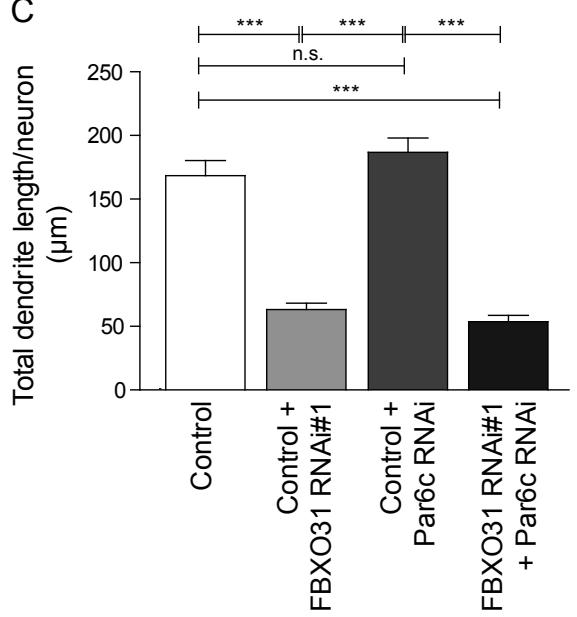

Figure 3.37: Par6c acts downstream of FBXO31-SCF in axon but not dendrite growth control. (A) Representative images of cerebellar granule neurons transfected with control vector, FBXO31 RNAi\#1, Par6c RNAi or FBXO31 RNAi\#1 and Par6c RNAi plasmids together with GFP and Bcl- $x_{L}$ expression plasmids at DIV 0 and analyzed at DIV 4. Arrowheads indicate cell bodies. Scale bar equals $50 \mu \mathrm{m}$. (B) Quantification of axon length of granule as neurons shown in (A). A total of 439 neurons were analyzed in 3 independent sets of experiments. Control: $269.5 \pm 10.19 \mu \mathrm{m}$, FBXO31 RNAi\#1: $169.9 \pm 6.37 \mu \mathrm{m}$, Par6c RNAi: $417.5 \pm 16.52 \mu \mathrm{m}$ and FBXO31 RNAi\#1+Par6c RNAi: 344.8 $\pm 15.39 \mu \mathrm{m}$. Values indicate mean \pm SEM (one-way ANOVA, $\left.{ }^{* * *} \mathrm{p}<0.001\right)$. (C) Quantification of total dendrite lengths of granule neurons as shown in (A). A total of 318 neurons were analyzed in 3 independent sets of experiments. Control: $168.4 \pm 11.93 \mu \mathrm{m}$, FBXO31 RNAi\#1: $63.28 \pm 5.03 \mu \mathrm{m}$, Par6c RNAi: $186.8 \pm 11.26 \mu \mathrm{m}$ and FBXO31 RNAi\#1+Par6c RNAi: $53.73 \pm 4.86 \mu \mathrm{m}$. Values indicate mean \pm SEM (one-way ANOVA, ${ }^{* * *} \mathrm{p}<0.001$ ).

while Cdc20-APC has been shown to promote dendrite growth and branching by targeting Id1 for degradation (Kim et al., 2009).

In the analysis of FBXO31 sequence, I identified eight D-box domains (Figure 3.38), which could be potential recognition sites of Cdh1. Moreover, FBXO31 levels 
oscillate during the cell cycle and show a maximum expression from late G2 to early G1 (Kumar et al., 2005). The timing of FBXO31 destruction is also consistent with the Cdh1-APC activation during cell cycle (Peters, 2006). Therefore I asked if FBXO31 could be a substrate targeted for degradation by Cdh1-APC.

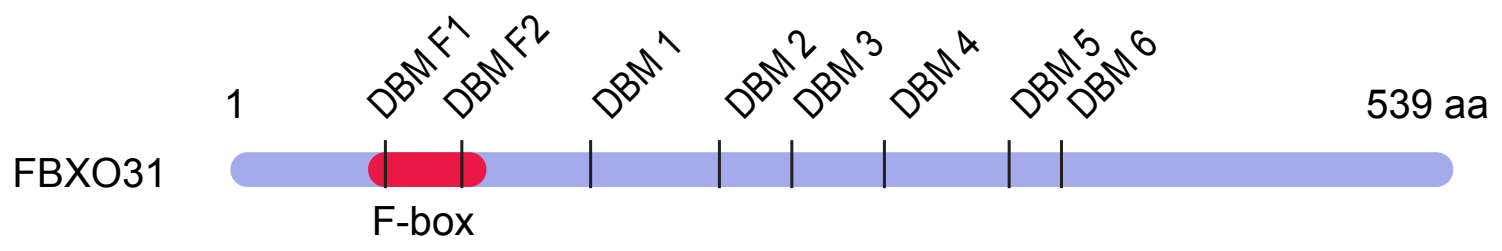

Figure 3.38: D-box motifs and their mutants in FBX031. Schematic showing eight D-box motifs, putative recognition site of Cdh1-APC, on FBXO31. Individual D-box motifs with consensus sequence RxxL were mutated to AxxA by site-directed mutagenesis. (DBM = D-box mutant)

\subsubsection{FBXO31 interacts with Cdh1}

To investigate FBXO31 as a potential interactor and target of Cdh1, I first addressed if FBXO31 and Cdh1 interact. For this, I transfected HEK 293T cells with mycFBXO31 and Flag-Cdh1 plasmids together or with respective control vectors. The cell lysates were subjected to immunoprecipitation with anti-Flag antibody and immunoblotted for FBXO31. I found a specific interaction of FBXO31 with Cdh1 (Figure 3.39).

In order to identify the D-box domain responsible for interaction of FBXO31 with Cdh1, I transfected HEK 293T cells with Flag-Cdh1 together with mycFBXO31 WT or with individual D-box mutants (mycFBXO31 DBM F1, F2, 1-6). The cell lysates were immunoprecipitated with anti-Flag antibody and immunoblotted for FBXO31. Although I found FBXO31 WT to interact with Cdh1, I also observed all DBM's to interact with Cdh1 (Figure 3.40), suggesting that neither of the D-box motifs is a bona fide one or a combination of various D-box motifs is required, which needs further investigation. 


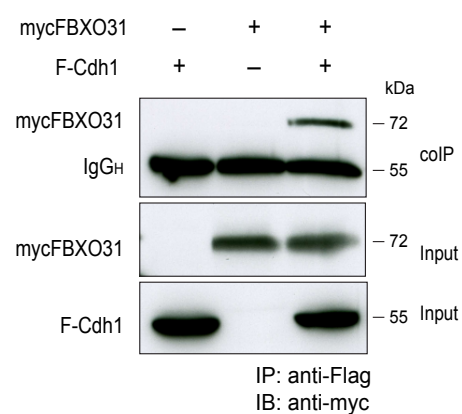

Figure 3.39: FBXO31 interacts with Cdh1. HEK 293T cells were transfected with mycFBXO31 and Flag-Cdh1 and respective control vectors. The cell lysates were subjected to immunoprecipitation with anti-Flag antibody and immunoblotted for FBXO31. Inputs represent $5 \%$ of total cell lysate prior to immunoprecipitation.
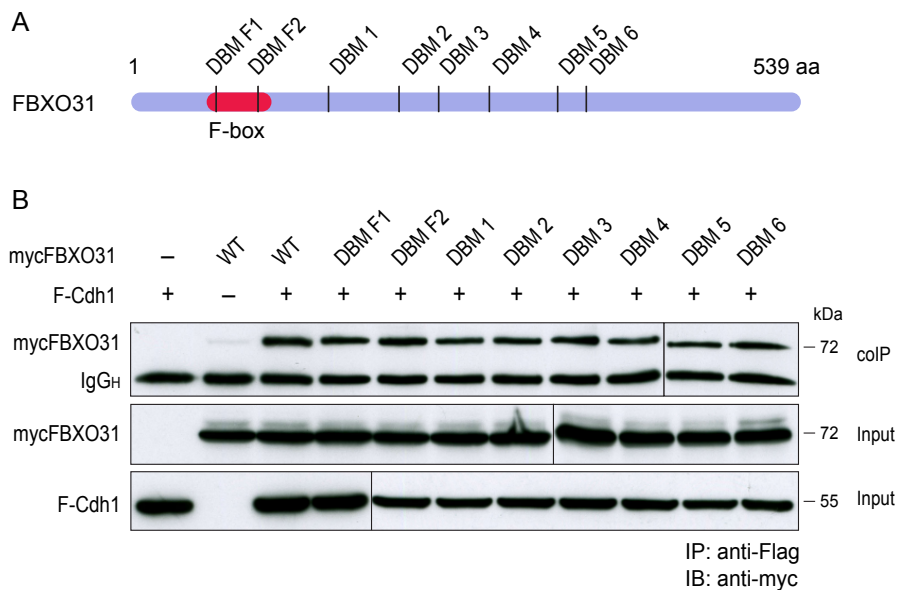

Figure 3.40: Cdh1 interacts with FBXO31 D-box mutants. (A) Schematic of FBXO31 DBM as shown in Figure 3.38. (B) HEK 293T cells were transfected with Flag-Cdh1 and mycFBXO31 WT or different D-box mutants as indicated. The cell lysates were subjected to immunoprecipitation with anti-Flag antibody and immunoblotted for FBXO31. Inputs represent $5 \%$ of total protein lysate prior to immunoprecipitation.

\subsubsection{Cdh1 regulates the stability of FBXO31}

To further characterize the interaction of FBXO31 and Cdh1, I examined if Cdh1 regulates the stability of FBXO31. To test this, Dr. Judith Stegmüller transfected HEK 293T cells with FBXO31 and Cdh1 expression plasmids together with 
Cdh1 RNAi or control plasmid. The cell lystes were immunoblotted for FBXO31. There was a significant increase in levels of FBXO31 upon knockdown of Cdh1 as compared to control lysates (Figure 3.41).

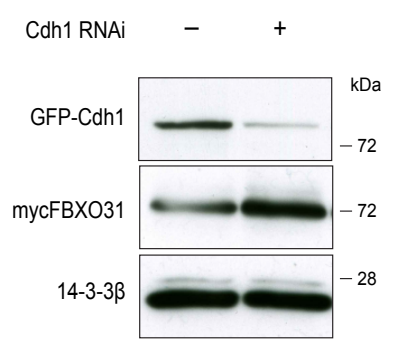

Figure 3.41: Cdh1 regulates stability of FBXO31. HEK 293T cells were transfected with GFPCdh1 and mycFBXO31 together with control or Cdh1 RNAi. The cell lysates were immunoblotted for FBXO31 and Cdh1.

\subsubsection{FBXO31 acts downstream of Cdh1 in control of axon growth}

Cdh1-APC has been previously reported as a suppressor of axon growth in neurons by targeting SnoN, Id2 and Smurf1 for degradation (Lasorella et al., 2006; Stegmüller et al., 2006; Kannan et al., 2012). Since I identified FBXO31 as an axon growth promoter in my study, I investigated if FBXO31 acts downstream of Cdh1APC in axon growth regulation. To address this, I performed epistasis analysis and transfected granule neurons with FBXO31 RNAi or Cdh1 RNAi or both plasmids and respective control vectors at DIV 0 and analyzed these neurons for their axon lengths at DIV 4. I found a significant reduction in axon length upon FBXO31 knockdown and as reported earlier by Konishi and colleagues (Konishi et al., 2004), a significant increase in axon length upon knockdown of Cdh1. In FBXO31/Cdh1 double knockdown conditions, I observed short axon phenotype suggesting that FBXO31 phenotype prevails (Figure 3.42A,B). This data suggests that FBXO31 acts downstream of Cdh1 in control of axon growth in CGNs. 

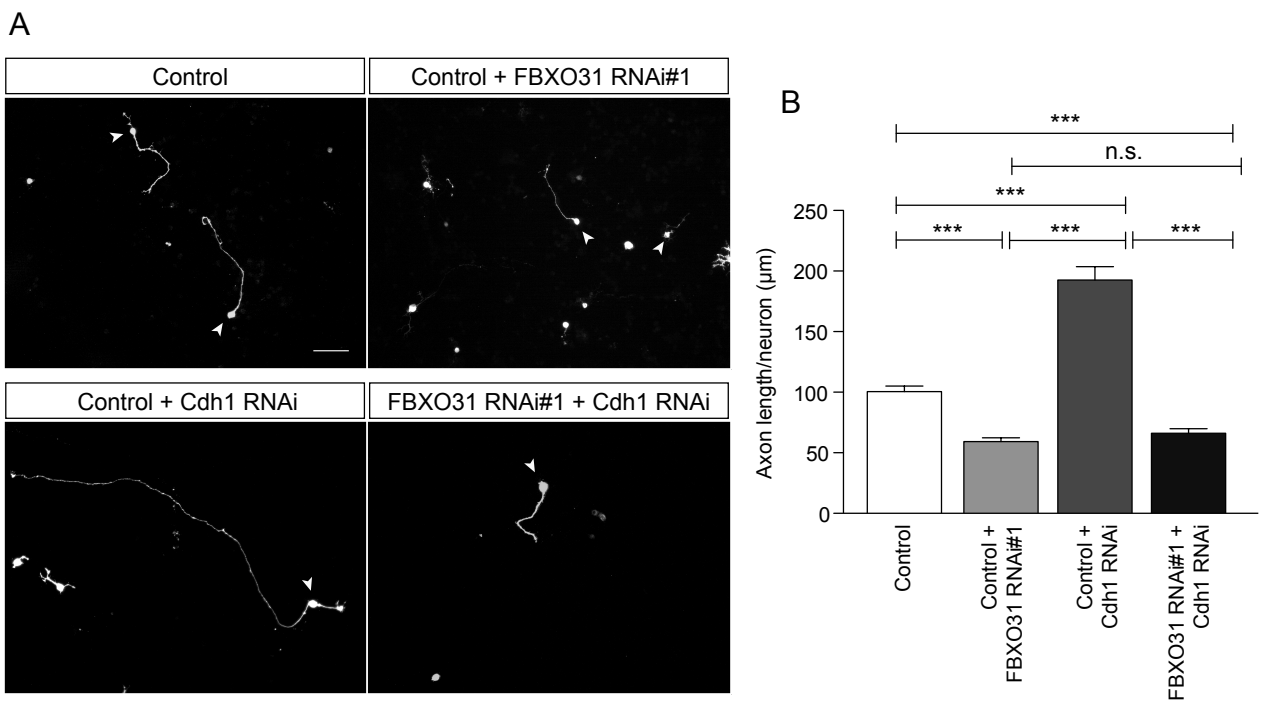

Figure 3.42: FBXO31 acts downstream of $\mathrm{Cdh} 1$ in control of axon growth in cerebellar granule neurons. (A) Representative images of cerebellar granule neurons transfected with control vector, FBXO31 RNAi\#1, Cdh1 RNAi or FBXO31 RNAi\#1 with Cdh1 RNAi together with GFP and Bcl- $x_{L}$ expression plasmids. Arrowheads indicate cell bodies. Scale bar equals $50 \mu \mathrm{m}$. (B) Quantification of axon length of granule neurons as shown in (A). A total of 560 neurons were analyzed in 3 independent sets of experiments. Control: $100.5 \pm 4.62 \mu \mathrm{m}$, FBXO31 RNAi\#1: $59.35 \pm 3.13 \mu \mathrm{m}$, Cdh1 RNAi: $192.5 \pm 11.13 \mu \mathrm{m}$, FBXO31 RNAi\#1+Cdh1 RNAi: $66.11 \pm 3.78 \mu \mathrm{m}$. Values indicate mean \pm SEM (ANOVA, ${ }^{* \star *} p<0.001$ ). 
Science never solves a problem without creating ten more.

George Bernard Shaw

\section{Discussion}

Neuronal development is a tightly regulated process that involves an interplay between extrinsic and intrinsic cues. Extrinsic factors signal via the intracellular regulators to control cytoskeletal dynamics, which are critical during neuronal development. Spatial and temporal regulation of protein turnover by the ubiquitin proteasome system has emerged as an important cell-intrinsic pathway for the control of neuronal morphogenesis.

The SCF E3 ubiquitin ligase, in particular the substrate recruiting interchangeable subunits F-box proteins, have surfaced as modulators of neuronal morphogenesis. A quest for the role of F-box proteins in post-mitotic neurons has recently gained momentum. Although some of the F-box proteins have been implicated in various stages of neuronal development including progenitor proliferation, neural differentiation, neuronal migration, axon/dendrite growth and synapotogenesis, the function of a large number of brain-enriched F-box proteins remains elusive. My study provides the first evidence for the role of a novel brain-enriched F-box protein FBXO31 in neurons.

\subsection{Role of FBXO31 in neuronal morphogenesis}

\subsubsection{FBXO31 is a centrosomal E3 ubiquitin ligase}

The F-box protein FBXO31 was initially characterized as a breast tumor suppressor and a cell cycle regulator (Kumar et al., 2005; Santra et al., 2009). Kumar and colleagues identified FBXO31 expression ubiquitous with a pronounced enrich- 
ment in the human brain (Kumar et al., 2005). This supports my data showing relative enrichment of FBXO31 in various regions of the rat brain. Although in the study, Kumar and colleagues describe FBXO31 as a potential regulator of cell cycle, they do not ascribe any function of FBXO31 in the nervous system. Given an enrichment of FBXO31 in brain, I sought to elucidate its function during neuronal development.

I found FBXO31 to be localized at the centrosome in mitotic cells (HEK 293T cells) and in post-mitotic hippocampal and cerebellar granule neurons. Centrosome is the seat for microtubule nucleation and thus co-ordinates the cytoskeletal machinery in the cells (Higginbotham and Gleeson, 2007). Being a signaling hub, regulated protein turnover at the centrosome seems essential. In this context, E3 ubiquitin ligases such as Cdh1-APC and Cdc20-APC associate with the centrosome in the cycling cell to co-ordinate various events during cell cycle transitions (Kallio et al., 2002; Zhou et al., 2003). In addition to this, essential scaffold proteins of the SCF complex, Skp1 and Cul1, are also localized at the centrosome during the mitotic cycle (Freed et al., 1999).

Interestingly, the E3 ubiquitin ligase Cdc20-APC is localized at the centrosome in neurons and is required for dendrite growth (Kim et al., 2009; Puram et al., 2011). Another E3 ubiquitin ligase Parkin, associated with Parkinson's disease, is also recruited to the centrosome in response to proteasome inhibition (Zhao et al., 2003; Jiang et al., 2008), suggesting an essential role of centrosomal E3 ubiquitin ligases in both neuronal development and disease. FBXO31 localization at the centrosome also suggests its possible function in neuronal morphogenesis that could have potential implication in neurological disorder. This is discussed further in the subsequent sections. 


\subsubsection{FBXO31-SCF promotes of axon and dendrite growth in neurons}

In neurons, axon and dendrite growth depends on microtubule nucleation at the centrosome. This is further facilitated by the transport of specific cargos to either axon or dendrite by molecular motors including dyneins and kinesins (Hirokawa et al., 2010). FBXO31-SCF localization at the centrosome prompted me to investigate its role in axon and dendrite growth. I found that while FBXO31 knockdown using an RNAi-approach, reduced axon and dendrite growth, overexpression of FBXO31 accelerated growth in neurons. In addition, I found that FBXO31-SCF ligase activity is essential for its effect on both axon and dendrite growth as the mutant (FBXO31 $\Delta \mathrm{F})$ that does not interact with Skp1 and Cul1, does not show growth promoting phenotype. Thus, centrosomal FBXO31 acts as a promoter of axon and dendrite growth in neurons.

FBXO31-SCF joins the league of centrosomal E3 ligase Cdc20-APC in the control of neuronal morphogenesis. Cdc20-APC promotes dendrite growth by ubiquitination of centrosomal protein Id1 (Kim et al., 2009). Cdc20-APC activity at the centrosome is regulated by phosphorylation at $\operatorname{Ser} 51$ by $\operatorname{CamKII} \beta$, which leads to dispersion of $\mathrm{Cdc} 20$ from the centrosome and retraction of the dendrites $(\mathrm{Pu}-$ ram et al., 2011). While Cdc20-APC regulates dendrite elaboration, it does not affect axon growth (Kim et al., 2009). This suggests a defined role of Cdc20-APC on neuronal morphogenesis. Unlike Cdc20-APC, FBXO31 regulates both axon and dendrite growth suggesting a role of FBXO31 in remodeling the overall morphology of neurons.

Similar to FBXO31, another E3 ubiquitin ligase NEDD4 regulates both axon and dendrite morphogenesis by targeting different substrates. NEDD4 is a HECT E3 ubiquitin ligase that is predominantly expressed in retinal ganglion cell growth cone and inhibits its terminal branching by regulating PTEN levels (Drinjakovic et al., 2010). PTEN is a negative regulator of the PI3K signaling pathway that is essential for axon branching. Moreover, decrease in PTEN rescues the axon 
branching defect caused by NEDD4 inhibition. In addition to this, NEDD4-1 also plays a critical role in dendrite morphogenesis (Kawabe et al., 2010). NEDD4-1 knockout mice show an impaired dendrite development. The complex consisting of NEDD4-1, the serine/threonine kinase TNIK and Rap2A mediates ubiquitination of Rap2A. This renders Rap2A effector kinases TNIK and MINK inactive and results in dendrite growth stimulation. Similar to NEDD4, FBXO31-SCF might target different proteins to regulate axon and dendrite growth. This question is addressed in detail later in the discussion.

In support of the observation that FBXO31 regulates neuronal morphogenesis in vitro, my in vivo electroporation results underscore a crucial role of FBXO31 in dendrite development. I observe that RNAi-mediated knockdown of FBXO31 in vivo results in shorter dendrite length, suggesting an important function of FBXO31-SCF in the developing brain. The in vivo analysis is limited to dendrite growth as the assessment of axon growth was not feasible since the axons fasciculate together in the molecular layer as untraceable fibers.

\subsubsection{FBXO31-SCF regulates neuronal polarity}

Extracellular cues trigger intracellular signaling cascades that break the initial neuronal symmetry leading to axon-dendrite polarization. Centrosome-mediated microtubule reorganization has been implicated in early polarization events in neurons (Higginbotham and Gleeson, 2007). In fact, localization of the centrosome in the neuron determines the axonal fate of a neurite (Zmuda and Rivas, 1998; de Anda et al., 2005, 2010). It has also been suggested that the centrosome is coupled with the Golgi complex at the base of the future axon (de Anda et al., 2005) and thus promote directed-trafficking of Golgi-derived vesicle (Sütterlin and Colanzi, 2010).

FBXO31-SCF localization at the centrosome prompted me to investigate its role in neuronal polarization. During my analysis of cerebellar granule neurons for axon and dendrite growth, I observed a large proportion of non-polarized neurons. This analysis was largely based on neurite measurements that established a ratio of 
the longest and the second longest process length. As axons undergo rapid elongation as compared to dendrites, I considered a neuron to be polarized when the length of longest process was at least twice as long as the second longest process.

Furthermore, molecular examination of hippocampal neurons with the axon initial segment protein ankyrinG revealed that FBXO31-SCF is required for axonal specification. While RNAi-mediated knockdown of FBXO31 results in a significant increase of neurons with no axon, overexpression of FBXO31 promotes a multipleaxon phenotype. This was again dependent on ligase activity of the FBXO31SCF complex, as FBXO31 $\Delta \mathrm{F}$ did not show a multiple-axon phenotype. These results provide the first evidence of centrosomal F-box protein regulating neuronal polarization.

\subsubsection{FBXO31-SCF is essential for neuronal migration in the developing cerebellum}

During neuronal migration in the developing cortex or cerebellum, the centrosome is typically located in front of the nucleus in the direction of migration (Hatten, 1999). Elegant time-lapse imaging by Solecki and colleagues revealed that glialguided neuronal migration is a two-step process (Solecki et al., 2004). In the first step, the leading process extends forward along the substrate, followed by centrosomal movement into the leading process. In the second step, the nucleus translocates towards the centrosome. In this context, the centrosome links the microtubule-based pulling forces generated within the extending process and the network of microtubules that surrounds the nucleus. A disruption of the microtubule network could uncouple the centrosomal and nuclear movement resulting in impaired migration of the neuron. This is observed in neurons from the doublecortin (DCX)-deficient mice (Koizumi et al., 2006). DCX is a microtubule-stabilizing protein that is localized at the centrosome and the perinuclear cage (Tanaka et al., 2004). In DCX-deficient neurons, the forces generated in the leading process by the microtubules that move the centrosome are uncoupled from the perinuclear cage microtubule-array. This re- 
sults in failed nuclear transport and compromised migration (Koizumi et al., 2006). Thus, the centrosome and the microtubule network are essential for neuronal migration.

In my in vivo analysis of the developing cerebellum (performed in collaboration with Dr. Judith Stegmüller) I found reduced dendritic growth upon FBXO31 knockdown. I also observed an impaired migration of neurons into the internal granule layer. The majority of the neurons were stalled in the molecular layer, suggesting an important role of centrosomal FBXO31-SCF in neuronal migration. Whether FBXO31 regulates migration by coupling the centrosome and the microtubule network needs further investigation.

\subsection{Insights into FBXO31-SCF-regulated neuronal morphogenesis}

\subsubsection{Par6 is a novel substrate of FBXO31-SCF in control of axon growth}

The localization of FBXO31 at the centrosome led me to discover members of the par complex as interaction partners. As discussed in the introduction, the par polarity complex primarily consists of Par6 (Par6 $\alpha$, Par6 $\beta$ and Par6 $\gamma$ ), aPKC $(\mathrm{PKC} \iota$ or $\mathrm{PKC} \zeta)$ and Par3. In my screen for novel interaction partners, I identified Par6c (also called Par6 $\alpha$ ), Par6 $\beta, \mathrm{PKC} \iota$ and $\mathrm{PKC} \zeta$ to interact with $\mathrm{FBXO} 31$. As Par6c represents the major Par6 protein in the nervous tissue (Assémat et al., 2008), I restricted my investigation to Par6c. Moreover, I did not observe an interaction of FBXO31 with Par3b, suggesting that FBXO31 interacts with the core members of the polarity complex proteins. I, together with my colleague Nicola Schwedhelm-Domeyer, also identified Par6c as a novel substrate of FBXO31-SCF. I found FBXO31-SCF to polyubiquitinate Par6c and target it for degradation via the $26 \mathrm{~S}$ proteasome. 
Although Par6, Par3 and aPKC act together in the control of epithelial and neuronal cell polarity that is highly conserved amongst various species (Wodarz, 2002), recent evidence supports the notion that these players can act independently of each other. For example, Par6 interacts with aPKC in the control of spine morphogenesis by regulating the activity of p190 RhoGAP (Zhang and Macara, 2008). This function of Par6/aPKC is independent of Par3. Similarly, the same group reported the requirement of Par3 for spine morphogenesis in hippocampal neurons by spatially restricting Rac GEF Tiam1 to dendritic spines. This action of Par3 is independent of aPKC (Zhang and Macara, 2006). Thus, the interaction of FBXO31 with Par6/aPKC and not Par3 could have a more defined function that would need further investigation.

Par6c and aPKC are predominant centrosomal proteins. They relay the information from the extrinsic cues to cytoskeletal proteins. Par6 directly binds to Rho GTPases Cdc42 and Rac1 in their active GTP-bound form (Munro, 2006). Binding of GTP-bound Cdc42 or Rac1 activates the Par/aPKC complex, whoch promotes the phosphorylation of aPKC target proteins such as Par3 to promote cellular polarization (Assémat et al., 2008). Moreover, Par6 also recruits Smurf1 which, in turn, mediates the degradation of small GTPase and growth inhibitor RhoA. This facilitates growth by formation of actin-rich filopodia-like protrusions (Wang et al., 2003). Given that Par6c is degraded by FBXO31-SCF, it remains to be tested whether the function of FBXO31 is dependent on small GTPases such as Cdc42, Rac1 or RhoA.

The Par/aPKC complex has been well established for its function in neuronal polarization (Shi et al., 2003; Nishimura et al., 2004, 2005; Schwamborn et al., 2007; Yi et al., 2010; Cheng et al., 2011). As the neurons polarize, Par6, Par3 and aPKC are enriched at the tip of the nascent axon (Shi et al., 2003). During the establishment of neuronal polarity and axonal identity, an extrinsic signal leads to local activation of PI3K at the tip of the nascent axon that results in recruitment of Par3, Par6 and aPKC. Simultaneously, PI3K activates aPKC, which facilitates 
differential regulation of the cytoskeletal dynamics at the growth cone and promotes axon growth (Shi et al., 2003). Whether FBXO31, Par6c and PI3K act in a pathway to regulate axon growth needs further investigation.

Other extrinsic cues also converge on the Par/aPKC complex to induce neuronal polarization. For example, BDNF promotes axon growth by recruiting Smurf1 to the growth cone (Cheng et al., 2011). Non-phosphorylated Smurf1 at the tip of the neurites promotes Par6 ubiquitination. Upon stimulation with BDNF, Smurf1 is phosphorylated by PKA, which induces a switch to promote RhoA degradation and stabilizes Par6. This stimulates the extension of the nascent axon.

A similar study identified TGF $\beta$ as an extrinsic cue that regulates axon specification and extension by mediating site-specific phosphorylation of Par6c (Yi et al., 2010). The authors found that TGF $\beta$ RI and Par6c to colocalize at the growth cones in undifferentiated neurites, particularly in filopodial protrusions. In presence of TGF $\beta$, Par6c phosphorylation by TGF $\beta$ RII recruits Smurf1 to the growth cones, which in turn promotes the proteasomal degradation of RhoA (Ozdamar et al., 2005). In this manner, the local inactivation of RhoA promotes axon growth.

While Par6 activity at the growth cone is essential for axon growth, its localization at the centrosome is necessary during neuronal migration and axon extension. At the centrosome, Par6 is involved in the assembly of the peri-nuclear cage along with an array of microtubules. Overexpression of Par6c results in disruption of the peri-nuclear cage that causes the centrosomal proteins $\gamma$-tubulin and $\mathrm{PKC} \zeta$ to disperse away from the centrosome (Solecki et al., 2004). This inhibits the migration of granule neurons into the internal granule layer and also results in retardation of axon growth. Par6 also functions to recruit PCM1 (pericentriolar material 1) and dynactin subunit p150Glued to the centrosome (Kodani et al., 2010). Depletion of Par6 results in mislocalization of p150Glued and PCM1 that are critical for microtubule anchoring at the centrosome.

The question that arises is how does localization of Par6c regulate axon growth? While Smurf1 phosphorylation at the growth cone induces a shift in substrate pref- 
erence from Par6 to RhoA, a stoichiometry of Par6/RhoA level would eventually determine the extent of axon growth. At the centrosome, Par6 acts as a scaffold protein and recruits other centrosomal proteins. Here, a restricted activity of Par6c would be necessary to switch between different developmental stages of neurons from polarization to migration to axon growth. In my study, I identify that centrosomal E3 ubiquitin ligase FBXO31-SCF degrades Par6c. Thus, FBXO31-SCF could provide a necessary check to maintain a balance of the Par6c activity at the centrosome.

In this context, I performed functional studies with Par6c gain-of-function and loss-of-function, and identified Par6c as a suppressor of axon growth. While Par6c overexpression resulted in shorter axons, knockdown of Par6 promoted axon growth. As expected, Par6c overexpression also resulted in altered polarity. While Par6c seems to be essential for axon growth, I did not find defects in dendrite growth with altered levels of Par6c. In addition, my epistasis experiments identify Par6c to act downstream of FBXO31 in control of axon growth (Figure 4.1). Two inferences can be made from this observation. First, since Par6c does not regulate dendrite length, FBXO31 might target other unidentified substrates to regulate dendrite length. Second, Par6c is not essential for initial morphogenesis of dendrites but is rather required in the later stages of spine morphogenesis as shown by Zhang and Macara (Zhang and Macara, 2008). It remains elusive how Par6c is translocated to the dendrites and what features other than spine development, Par6c is involved in.

Elucidation of the centrosomal FBXO31-SCF/Par6 signaling implies that both FBXO31-SCF and Par6 may exert functions beyond the control of axonal morphogenesis. FBXO31, localized at the centrosome in mitotic cells, oscillates during cell cycle (Kumar et al., 2005). In response to DNA damage, FBXO31 mediates degradation of cyclin D1 and induces G1 arrest (Santra et al., 2009). Moreover, FBXO31 acts as a breast tumor suppressor, whereas Par6 levels are elevated in breast cancer (Kumar et al., 2005; Nolan et al., 2008). This scenario points to a conserved pathway between the cell biology of cancer cells and the axon development in neurons. 


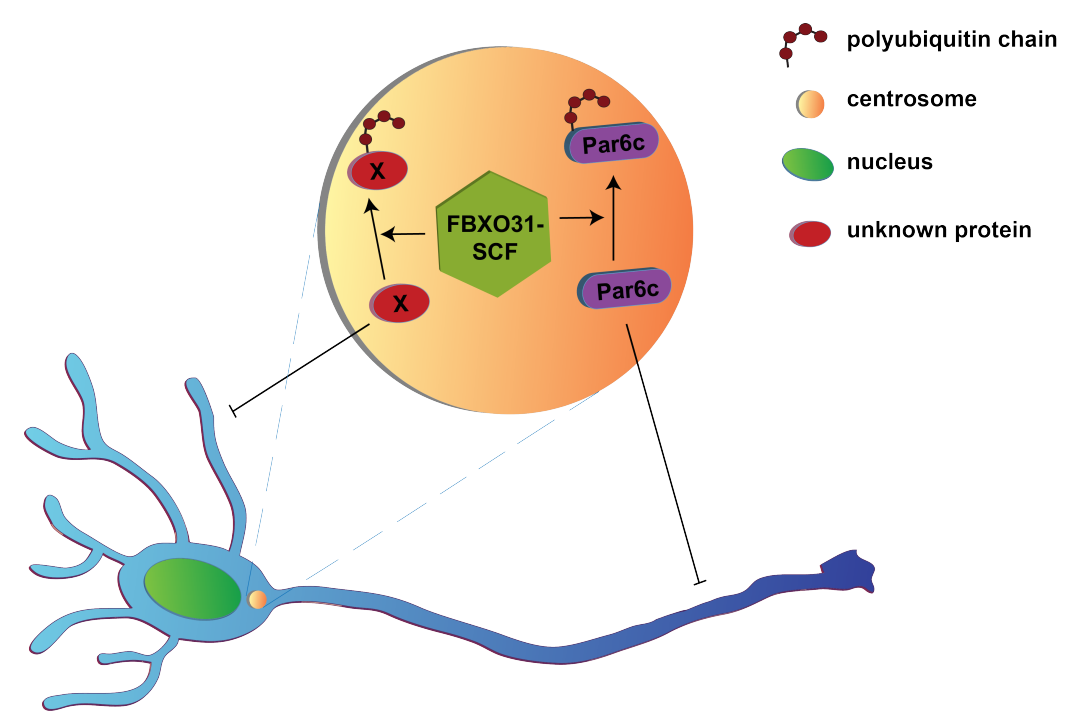

Figure 4.1: FBXO31-SCF promotes axon and dendrite growth in neurons. The E3 ubiquitin ligase FBXO31-SCF complex is localized at the centrosome in neurons. It promotes polyubiquitination and degradation of Par6c to promote the axon growth. FBXO31-SCF also mediates the ubiquitination of yet unidentified substrate to regulate dendrite morphogenesis.

\subsubsection{FBXO31 is a novel interaction partner of E3 ubiquitin ligase Cdh1-APC}

Cdh1-APC has been previously identified as a suppressor of axon growth. RNAi-mediated knockdown of Cdh1 in neurons triggers axon growth and disrupts parallel fiber fasciculation in the developing cerebellum (Konishi et al., 2004). In the context of axon growth, Cdh1 targets SnoN, Id2 and Smurf1 for degradation (Lasorella et al., 2006; Stegmüller et al., 2006; Kannan et al., 2012). While recent evidences provide a mechanistic insight into Cdh1-mediated axon growth regulation, it is conceivable that Cdh1-APC may act via additional pathways to regulate axon growth.

In my study, I identified FBXO31 as a novel interaction partner of Cdh1. I postulated an interaction of FBXO31 with Cdh1, owing to multiple putative D-boxes on FBXO31. D-boxes are recognition motifs that are recognized by Cdh1 on target substrates, which are subsequently recruited to the APC complex for ubiquitination 
(Peters, 2006). As FBXO31 harbors eight D-box domains, I hypothesized that Cdh1 interacts with FBXO31 via these D-box domains and targets it for degradation. Unlike what I expected, the D-box mutants of FBXO31 interact with Cdh1, suggesting that either the interaction is D-box-independent as none of the putative D-boxes is a bona fide one, or Cdh1 recognizes FBXO31 via multiple D-boxes. A recent report provides evidence that Cdh1 recognizes multiple D-boxes in Smurf1 to promote its degradation (Kannan et al., 2012).

In my epistasis analysis, I also observe FBXO31 to act downstream of Cdh1 in control of axon growth. Although, it needs to be further verified whether FBXO31 is a substrate of Cdh1, it seems likely that Cdh1 might degrade FBXO31 in control of axon growth. This is supported by evidence from the cell cycle as Cdh1 and FBXO31 levels oscillate alternately during cell cycle. FBXO31 levels peak from late G2 to early G1 with maximum activity during M-phase, whereas Cdh1 is activated at late anaphase and remains active until G1/S phase transition of the cell cycle (Kumar et al., 2005; Peters, 2006).

The Cdh1-APC/FBXO31-SCF signaling pathway may provide another checkpoint for the control of axon growth and elaboration during neuronal development. Furthermore, it remains to be tested whether Cdh1-APC/FBXO31-SCF/Par6c act in a linear pathway of axon growth control and whether this pathway would act in the parallel system in the biology of cell cycle.

Taken together, my study provides the first insight into the FBXO31-regulated events during neuronal development and uncovers FBXO31-SCF as a key regulator of neuronal morphogenesis. Furthermore, my study sets the stage for future research for the identification of novel substrates and other interaction partners to establish an FBXO31-SCF controlled signaling network in neurons and cell-cycle regulation. Interestingly, a recent report by Narayan and colleagues show that FBXO31 levels are downregulated in schizophrenic patients with short-term illness (Narayan et al., 2008), implicating a role of FBXO31 in schizophrenia. Therefore, genetic studies 
with systemic or conditional loss of FBXO31 would provide valuable information in context of neuronal developmental disorders such as schizophrenia. 
The important thing in science is not so much to obtain new facts as to discover new ways of thinking about them.

William Lawrence Bragg

\section{Summary and Outlook}

The formation of an intricate neuronal network requires processes that coordinate proliferation, migration and differentiation of neuronal cells. The incredible morphological changes observed in neurons as they migrate, extend processes or form synapses, imply a strictly regulated process of structural organization mediated by intracellular signaling cascades and cytoskeletal remodeling. Recently, the ubiquitin proteasome system particularly the E3 ubiquitin ligases have surfaced as essential regulators of neuronal morphogenesis.

My thesis describes the centrosomal E3 ubiquitin ligase FBXO31-SCF as a key regulator of several aspects of neuronal development. In this study, I used loss-offunction and gain-of-function approaches to establish a role of FBXO31-SCF in axon and dendrite growth. I found FBXO31-SCF stimulates both axonal and dendritic growth. In addition, I demonstrated that FBXO31-SCF regulates axonal identity of neurons and thus plays a critical role in neuronal polarization. Moreover, my in vivo results elucidated an indispensible role of FBXO31 in neuronal migration.

In order to gain a mechanistic insight into FBXO31-SCF-regulated events during neuronal development, I identified the polarity complex protein Par6c as a novel interaction partner and a bona fide substrate of FBXO31-SCF. In the functional assays, I identified Par6c as a suppressor of axon growth whereas it had no effect on dendrite length. In order to characterize the interaction between FBXO31 and Par6c, I performed epistasis analysis and identified that FBXO31 acts upstream of Par6c in the regulation of axon growth. This effect of FBXO31/Par6 pathway was specific to axon growth and did not affect dendrite length. Although, it needs to be 
tested whether FBXO31-SCF/Par6c pathway would regulate neuronal polarization and migration, it would be interesting future research to identify novel substrates of FBXO31-SCF to explain its effect on dendrite development.

In my study, I also found Cdh1-APC, an E3 ubiqutin ligase, as a novel interaction partner and regulator of FXBO31-SCF stability. Cdh1-APC exerts an inhibitory effect on axon growth by targeting SnoN, Id2 and Smurf1 for degradation. Epistasis experiments revealed that FBXO31 acts downstream of Cdh1 in controlling axon growth. While it remains to be verified whether Cdh1-APC targets FBXO31 for degradation, it would be an exciting area to explore whether Cdh1APC/FBXO31-SCF/Par6c act in a linear pathway in regulating axon growth.

Recently FBXO31 has been implicated in schizophrenia where FBXO31 expression was found to be downregulated in patients with short-term illness. In future, the use of systemic and conditional knockout animals would provide valuable insights into neurodevelopmental disorders such as schizophrenia and may open up avenues for translational research. 


\section{Bibliography}

Almeida, A., Bolaños, J. P. and Moreno, S. (2005). Cdh1/Hct1-APC is essential for the survival of postmitotic neurons. The Journal of Neuroscience 25, 8115-21.

Anthony, T. E., Klein, C., Fishell, G. and Heintz, N. (2004). Radial glia serve as neuronal progenitors in all regions of the central nervous system. Neuron 41, 881-90.

Anton, E. S., Kreidberg, J. a. and Rakic, P. (1999). Distinct functions of alpha3 and alpha(v) integrin receptors in neuronal migration and laminar organization of the cerebral cortex. Neuron 22, 277-89.

Anton, E. S., Marchionni, M. a., Lee, K. F. and Rakic, P. (1997). Role of GGF/neuregulin signaling in interactions between migrating neurons and radial glia in the developing cerebral cortex. Development 124, 3501-10.

Araújo, S. J. and Tear, G. (2003). Axon guidance mechanisms and molecules: lessons from invertebrates. Nature Reviews Neuroscience 4, 910-22.

Arimura, N. and Kaibuchi, K. (2007). Neuronal polarity: from extracellular signals to intracellular mechanisms. Nature Reviews Neuroscience 8, 194-205.

Assémat, E., Bazellières, E., Pallesi-Pocachard, E., Le Bivic, A. and Massey-Harroche, D. (2008). Polarity complex proteins. Biochimica et Biophysica Acta 1778, 614-30.

Ballas, N. and Mandel, G. (2005). The many faces of REST oversee epigenetic programming of neuronal genes. Current Opinion in Neurobiology 15, 500-6.

Barnes, A. P. and Polleux, F. (2009). Establishment of axon-dendrite polarity in developing neurons. Annual Review of Neuroscience 32, 347-81.

Biben, C., Stanley, E., Fabri, L., Kotecha, S., Rhinn, M., Drinkwater, C., Lah, M., Wang, C. C., Nash, A., Hilton, D., Ang, S. L., Mohun, T. and Harvey, R. P. (1998). Murine cerberus homologue mCer-1: a candidate anterior patterning molecule. Developmental Biology 194, 135-51.

Bixby, J. L. and Harris, W. A. (1991). Molecular mechanisms of axon growth and guidance. Annual Review of Cell Biology 7, 117-59. 
Branzei, D. and Foiani, M. (2008). Regulation of DNA repair throughout the cell cycle. Nature Reviews Molecular Cell Biology 9, 297-308.

Butler, M. G., Dagenais, S. L., Garcia-Perez, J. L., Brouillard, P., Vikkula, M., Strouse, P., Innis, J. W. and Glover, T. W. (2012). Microcephaly, intellectual impairment, bilateral vesicoureteral reflux, distichiasis, and glomuvenous malformations associated with a 16q24.3 contiguous gene deletion and a Glomulin mutation. American Journal of Medical Genetics Part A 158A, 839-49.

Cameron, R. S. and Rakic, P. (1991). Glial cell lineage in the cerebral cortex: a review and synthesis. Glia 4, 124-37.

Casazza, A., Fazzari, P. and Tamagnone, L. (2007). Semaphorin Signals in Cell Adhesion and Cell Migration: Functional Role and Molecular Mechanisms. Advances in Experimental Medicine and Biology 600, 90-108.

Chédotal, A. and Rijli, F. M. (2009). Transcriptional regulation of tangential neuronal migration in the developing forebrain. Current Opinion in Neurobiology 19, 139-45.

Chen, X., Wang, X., Sun, C., Chen, Q., O'Neill, F. A., Walsh, D., Fanous, A. and Kendler, K. S. (2008). FBXL21 association with schizophrenia in Irish family and case-control samples. American Journal of Medical Genetics Part B 147B, 1231-7.

Cheng, P.-1., Lu, H., Shelly, M., Gao, H. and Poo, M.-m. (2011). Phosphorylation of E3 ligase Smurf1 switches its substrate preference in support of axon development. Neuron 69, 231-43.

Cohen, P. and Tcherpakov, M. (2010). Will the ubiquitin system furnish as many drug targets as protein kinases? Cell 143, 686-93.

Costa, M. R., Wen, G., Lepier, A., Schroeder, T. and Götz, M. (2008). Par-complex proteins promote proliferative progenitor divisions in the developing mouse cerebral cortex. Development 135, 11-22.

Craig, a. M. and Banker, G. (1994). Neuronal polarity. Annual Review of Neuroscience $17,267-310$.

de Anda, F. C., Meletis, K., Ge, X., Rei, D. and Tsai, L.-H. (2010). Centrosome motility is essential for initial axon formation in the neocortex. The Journal of Neuroscience 30 , 10391-406.

de Anda, F. C., Pollarolo, G., Da Silva, J. S., Camoletto, P. G., Feiguin, F. and Dotti, C. G. (2005). Centrosome localization determines neuronal polarity. Nature 436, 704-8. 
de la Torre-Ubieta, L. and Bonni, A. (2011). Transcriptional regulation of neuronal polarity and morphogenesis in the mammalian brain. Neuron 72, 22-40.

Dent, E. W. and Gertler, F. B. (2003). Cytoskeletal dynamics and transport in growth cone motility and axon guidance. Neuron 40, 209-27.

Di Fonzo, a., Dekker, M. C. J., Montagna, P., Baruzzi, a., Yonova, E. H., Correia Guedes, L., Szczerbinska, a., Zhao, T., Dubbel-Hulsman, L. O. M., Wouters, C. H., de Graaff, E., Oyen, W. J. G., Simons, E. J., Breedveld, G. J., Oostra, B. a., Horstink, M. W. and Bonifati, V. (2009). FBXO7 mutations cause autosomal recessive, early-onset parkinsonian-pyramidal syndrome. Neurology 72, 240-5.

Dijkhuizen, P. A. and Ghosh, A. (2005). Regulation of dendritic growth by calcium and neurotrophin signaling. Progress in Brain Research 147, 17-27.

Dindot, S. V., Antalffy, B. A., Bhattacharjee, M. B. and Beaudet, A. L. (2008). The Angelman syndrome ubiquitin ligase localizes to the synapse and nucleus, and maternal deficiency results in abnormal dendritic spine morphology. Human Molecular Genetics $17,111-8$.

Dotti, C. G., Sullivan, C. A. and Banker, G. A. (1988). The establishment of polarity by hippocampal neurons in culture. The Journal of Neuroscience 8, 1454-68.

Dreier, L., Burbea, M. and Kaplan, J. M. (2005). LIN-23-mediated degradation of betacatenin regulates the abundance of GLR-1 glutamate receptors in the ventral nerve cord of C. elegans. Neuron 46, 51-64.

Drinjakovic, J., Jung, H., Campbell, D. S., Strochlic, L., Dwivedy, A. and Holt, C. E. (2010). E3 ligase Nedd4 promotes axon branching by downregulating PTEN. Neuron $65,341-57$.

Etemad-Moghadam, B., Guo, S. and Kemphues, K. J. (1995). Asymmetrically distributed PAR-3 protein contributes to cell polarity and spindle alignment in early C. elegans embryos. Cell 83, 743-52.

Evans, T. a. and Bashaw, G. J. (2010). Axon guidance at the midline: of mice and flies. Current Opinion in Neurobiology 20, 79-85.

Famulski, J. K., Trivedi, N., Howell, D., Yang, Y., Tong, Y., Gilbertson, R. and Solecki, D. J. (2010). Siah regulation of Pard3A controls neuronal cell adhesion during germinal zone exit. Science 330, 1834-8.

Fishell, G. and Hatten, M. E. (1991). Astrotactin provides a receptor system for CNS neuronal migration. Development 113, 755-65. 
Fishell, G. and Kriegstein, A. R. (2003). Neurons from radial glia: the consequences of asymmetric inheritance. Current Opinion in Neurobiology 13, 34-41.

Frank, C. L. and Tsai, L.-H. (2009). Alternative functions of core cell cycle regulators in neuronal migration, neuronal maturation, and synaptic plasticity. Neuron 62, 312-26.

Freed, E., Lacey, K. R., Huie, P., Lyapina, S. a., Deshaies, R. J., Stearns, T. and Jackson, P. K. (1999). Components of an SCF ubiquitin ligase localize to the centrosome and regulate the centrosome duplication cycle. Genes \& Development 13, 2242-57.

Frescas, D. and Pagano, M. (2008). Deregulated proteolysis by the F-box proteins SKP2 and beta-TrCP: tipping the scales of cancer. Nature Reviews Cancer 8, 438-49.

Fu, A. K. Y., Hung, K.-w., Fu, W.-y., Shen, C., Chen, Y., Xia, J., Lai, K.-o. and Ip, N. Y. (2011). APC(Cdh1) mediates EphA4-dependent downregulation of AMPA receptors in homeostatic plasticity. Nature Neuroscience 14, 181-9.

García-Higuera, I., Manchado, E., Dubus, P., Cañamero, M., Méndez, J., Moreno, S. and Malumbres, M. (2008). Genomic stability and tumour suppression by the APC/C cofactor Cdh1. Nature Cell Biology 10, 802-11.

Gieffers, C., Peters, B. H., Kramer, E. R., Dotti, C. G. and Peters, J. M. (1999). Expression of the CDH1-associated form of the anaphase-promoting complex in postmitotic neurons. PNAS 96, 11317-22.

Goldberg, J. L., Espinosa, J. S., Xu, Y., Davidson, N., Kovacs, G. T. A. and Barres, B. A. (2002). Retinal ganglion cells do not extend axons by default: promotion by neurotrophic signaling and electrical activity. Neuron 33, 689-702.

Gong, B., Chen, F., Pan, Y., Arrieta-Cruz, I., Yoshida, Y., Haroutunian, V. and Pasinetti, G. M. (2010). SCFFbx2-E3-ligase-mediated degradation of BACE1 attenuates Alzheimer's disease amyloidosis and improves synaptic function. Aging Cell 9, $1018-31$.

Goto, E., Yamanaka, Y., Ishikawa, A., Aoki-Kawasumi, M., Mito-Yoshida, M., OhmuraHoshino, M., Matsuki, Y., Kajikawa, M., Hirano, H. and Ishido, S. (2010). Contribution of lysine 11-linked ubiquitination to MIR2-mediated major histocompatibility complex class I internalization. The Journal of Biological Chemistry 285, 35311-9.

Götz, M. and Huttner, W. B. (2005). The cell biology of neurogenesis. Nature Reviews Molecular Cell Biology 6, 777-88.

Greer, P. L., Hanayama, R., Bloodgood, B. L., Mardinly, A. R., Lipton, D. M., Flavell, S. W., Kim, T.-K., Griffith, E. C., Waldon, Z., Maehr, R., Ploegh, H. L., Chowdhury, 
S., Worley, P. F., Steen, J. and Greenberg, M. E. (2010). The Angelman Syndrome protein Ube3A regulates synapse development by ubiquitinating arc. Cell 140, 704-16.

Guardavaccaro, D., Kudo, Y., Boulaire, J., Barchi, M., Busino, L., Donzelli, M., Margottin-Goguet, F., Jackson, P. K., Yamasaki, L. and Pagano, M. (2003). Control of meiotic and mitotic progression by the $\mathrm{F}$ box protein beta-Trcp1 in vivo. Developmental Cell 4, 799-812.

Guo, S. and Kemphues, K. J. (1995). par-1, a gene required for establishing polarity in C. elegans embryos, encodes a putative Ser/Thr kinase that is asymmetrically distributed. Cell 81, 611-20.

Hara, T., Nakamura, K., Matsui, M., Yamamoto, A., Nakahara, Y., Suzuki-Migishima, R., Yokoyama, M., Mishima, K., Saito, I., Okano, H. and Mizushima, N. (2006). Suppression of basal autophagy in neural cells causes neurodegenerative disease in mice. Nature 441, 885-9.

Harmey, D., Smith, A., Simanski, S., Moussa, C. Z. and Ayad, N. G. (2009). The anaphase promoting complex induces substrate degradation during neuronal differentiation. The Journal of biological chemistry 284, 4317-23.

Harper, J. W., Burton, J. L. and Solomon, M. J. (2002). The anaphase-promoting complex: it's not just for mitosis any more. Genes \& Development 16, 2179-206.

Hart, M., Concordet, J. P., Lassot, I., Albert, I., del los Santos, R., Durand, H., Perret, C., Rubinfeld, B., Margottin, F., Benarous, R. and Polakis, P. (1999). The F-box protein beta-TrCP associates with phosphorylated beta-catenin and regulates its activity in the cell. Current Biology 9, 207-10.

Hatten, M. E. (1999). Central nervous system neuronal migration. Annual Review of Neuroscience 22, 511-39.

Hemmati-Brivanlou, a., Kelly, O. G. and Melton, D. a. (1994). Follistatin, an antagonist of activin, is expressed in the Spemann organizer and displays direct neuralizing activity. Cell 77, 283-95.

Herrero-Mendez, A., Almeida, A., Fernández, E., Maestre, C., Moncada, S. and Bolaños, J. P. (2009). The bioenergetic and antioxidant status of neurons is controlled by continuous degradation of a key glycolytic enzyme by APC/C-Cdh1. Nature Cell Biology $11,747-52$.

Hershko, A. and Ciechanover, A. (1998). The ubiquitin system. Annual Review of Biochemistry $67,425-79$. 
Hershko, A., Ciechanover, A. and Rose, I. A. (1979). Resolution of the ATP-dependent proteolytic system from reticulocytes: a component that interacts with ATP. PNAS $76,3107-10$.

Higginbotham, H. R. and Gleeson, J. G. (2007). The centrosome in neuronal development. Trends in Neurosciences 30, 276-83.

Hirokawa, N., Niwa, S. and Tanaka, Y. (2010). Molecular motors in neurons: transport mechanisms and roles in brain function, development, and disease. Neuron 68, 610-38.

Hoeck, J. D., Jandke, A., Blake, S. M., Nye, E., Spencer-Dene, B., Brandner, S. and Behrens, A. (2010). Fbw7 controls neural stem cell differentiation and progenitor apoptosis via Notch and c-Jun. Nature Neuroscience 13, 1365-72.

Hu, D., Qiao, X., Wu, G. and Wan, Y. (2011). The emerging role of APC/CCdh1 in development. Seminars in Cell \& Developmental Biology 22, 579-85.

Huang, H.-l., Zheng, W.-l., Zhao, R., Zhang, B. and Ma, W.-l. (2010). FBXO31 is downregulated and may function as a tumor suppressor in hepatocellular carcinoma. Oncology Reports 24, 715-20.

Huang, Y. and Mucke, L. (2012). Alzheimer mechanisms and therapeutic strategies. Cell $148,1204-22$.

Huber, A. B., Kolodkin, A. L., Ginty, D. D. and Cloutier, J.-F. (2003). Signaling at the growth cone: ligand-receptor complexes and the control of axon growth and guidance. Annual Review of Neuroscience 26, 509-63.

Huntwork, S. and Littleton, J. T. (2007). A complexin fusion clamp regulates spontaneous neurotransmitter release and synaptic growth. Nature Neuroscience 10, 1235-7.

Ikeda, F. and Dikic, I. (2008). Atypical ubiquitin chains: new molecular signals. 'Protein Modifications: Beyond the Usual Suspects' review series. EMBO Reports 9, 536-42.

Ikeuchi, Y., Stegmüller, J., Netherton, S., Huynh, M. A., Masu, M., Frank, D., Bonni, S. and Bonni, A. (2009). A SnoN-Ccd1 pathway promotes axonal morphogenesis in the mammalian brain. The Journal of Neuroscience 29, 4312-21.

Jandke, A., Da Costa, C., Sancho, R., Nye, E., Spencer-Dene, B. and Behrens, A. (2011). The F-box protein Fbw7 is required for cerebellar development. Developmental Biology $358,201-12$.

Jiang, Q., Ren, Y. and Feng, J. (2008). Direct binding with histone deacetylase 6 mediates the reversible recruitment of parkin to the centrosome. The Journal of Neuroscience 28, 12993-3002. 
Jin, J., Cardozo, T., Lovering, R. C., Elledge, S. J., Pagano, M. and Harper, J. W. (2004). Systematic analysis and nomenclature of mammalian F-box proteins. Genes \& Development 18, 2573-80.

Juo, P. and Kaplan, J. M. (2004). The anaphase-promoting complex regulates the abundance of GLR-1 glutamate receptors in the ventral nerve cord of C. elegans. Current Biology 14, 2057-62.

Kallio, M. J., Beardmore, V. a., Weinstein, J. and Gorbsky, G. J. (2002). Rapid microtubule-independent dynamics of Cdc20 at kinetochores and centrosomes in mammalian cells. The Journal of Cell Biology 158, 841-7.

Kanie, T., Onoyama, I., Matsumoto, A., Yamada, M., Nakatsumi, H., Tateishi, Y., Yamamura, S., Tsunematsu, R., Matsumoto, M. and Nakayama, K. I. (2012). Genetic reevaluation of the role of F-box proteins in cyclin D1 degradation. Molecular and Cellular Biology 32, 590-605.

Kannan, M., Lee, S.-J., Schwedhelm-Domeyer, N. and Stegmüller, J. (2012). The E3 ligase Cdh1-anaphase promoting complex operates upstream of the E3 ligase Smurf1 in the control of axon growth. Development 139, In press.

Kawabe, H., Neeb, A., Dimova, K., Young, S. M., Takeda, M., Katsurabayashi, S., Mitkovski, M., Malakhova, O. A., Zhang, D.-E., Umikawa, M., Kariya, K.-i., Goebbels, S., Nave, K.-A., Rosenmund, C., Jahn, O., Rhee, J. and Brose, N. (2010). Regulation of Rap2A by the ubiquitin ligase Nedd4-1 controls neurite development. Neuron 65 , $358-72$.

Kemphues, K. J., Priess, J. R., Morton, D. G. and Cheng, N. S. (1988). Identification of genes required for cytoplasmic localization in early C. elegans embryos. Cell 52, $311-20$.

Kerscher, O., Felberbaum, R. and Hochstrasser, M. (2006). Modification of proteins by ubiquitin and ubiquitin-like proteins. Annual Review of Cell and Developmental Biology 22, 159-80.

Kim, A. H., Puram, S. V., Bilimoria, P. M., Ikeuchi, Y., Keough, S., Wong, M., Rowitch, D. and Bonni, A. (2009). A centrosomal Cdc20-APC pathway controls dendrite morphogenesis in postmitotic neurons. Cell 136, 322-36.

Kim, S. and Chiba, A. (2004). Dendritic guidance. Trends in Neurosciences 27, 194-202.

Kipreos, E. T., Gohel, S. P. and Hedgecock, E. M. (2000). The C. elegans F-box/WDrepeat protein LIN-23 functions to limit cell division during development. Development 127, 5071-82. 
Kipreos, E. T. and Pagano, M. (2000). The F-box protein family. Genome Biology 1, REVIEWS3002.

Kodani, A., Tonthat, V., Wu, B. and Sütterlin, C. (2010). Par6 alpha interacts with the dynactin subunit p150 Glued and is a critical regulator of centrosomal protein recruitment. Molecular Biology of the Cell 21, 3376-85.

Koizumi, H., Higginbotham, H., Poon, T., Tanaka, T., Brinkman, B. C. and Gleeson, J. G. (2006). Doublecortin maintains bipolar shape and nuclear translocation during migration in the adult forebrain. Nature Neuroscience 9, 779-86.

Komatsu, M., Waguri, S., Chiba, T., Murata, S., Iwata, J.-i., Tanida, I., Ueno, T., Koike, M., Uchiyama, Y., Kominami, E. and Tanaka, K. (2006). Loss of autophagy in the central nervous system causes neurodegeneration in mice. Nature 441, 880-4.

Konishi, Y., Stegmüller, J., Matsuda, T., Bonni, S. and Bonni, A. (2004). Cdh1-APC controls axonal growth and patterning in the mammalian brain. Science 303, 1026-30.

Kuczera, T., Stilling, R. M., Hsia, H.-E., Bahari-Javan, S., Irniger, S., Nasmyth, K., Sananbenesi, F. and Fischer, A. (2011). The anaphase promoting complex is required for memory function in mice. Learning \& Memory 18, 49-57.

Kumar, R., Neilsen, P. M., Crawford, J., McKirdy, R., Lee, J., Powell, J. A., Saif, Z., Martin, J. M., Lombaerts, M., Cornelisse, C. J., Cleton-Jansen, A.-M. and Callen, D. F. (2005). FBXO31 is the chromosome 16q24.3 senescence gene, a candidate breast tumor suppressor, and a component of an SCF complex. Cancer Research 65, 11304-13.

Lai Wing Sun, K., Correia, J. P. and Kennedy, T. E. (2011). Netrins: versatile extracellular cues with diverse functions. Development 138, 2153-69.

Lamb, T. M., Knecht, a. K., Smith, W. C., Stachel, S. E., Economides, a. N., Stahl, N., Yancopolous, G. D. and Harland, R. M. (1993). Neural induction by the secreted polypeptide noggin. Science 262, 713-8.

Lasorella, A., Stegmüller, J., Guardavaccaro, D., Liu, G., Carro, M. S., Rothschild, G., de la Torre-Ubieta, L., Pagano, M., Bonni, A. and Iavarone, A. (2006). Degradation of Id2 by the anaphase-promoting complex couples cell cycle exit and axonal growth. Nature 442, 471-4.

Latres, E., Chiaur, D. S. and Pagano, M. (1999). The human F box protein beta-Trcp associates with the Cul1/Skp1 complex and regulates the stability of beta-catenin. Oncogene $18,849-54$. 
Lentz, S. I., Knudson, C. M., Korsmeyer, S. J. and Snider, W. D. (1999). Neurotrophins support the development of diverse sensory axon morphologies. The Journal of Neuroscience 19, 1038-48.

Levitan, D. J., Boyd, L., Mello, C. C., Kemphues, K. J. and Stinchcomb, D. T. (1994). par-2, a gene required for blastomere asymmetry in Caenorhabditis elegans, encodes zinc-finger and ATP-binding motifs. PNAS 91, 6108-6112.

Li, M., Shin, Y.-H., Hou, L., Huang, X., Wei, Z., Klann, E. and Zhang, P. (2008). The adaptor protein of the anaphase promoting complex Cdh1 is essential in maintaining replicative lifespan and in learning and memory. Nature Cell Biology 10, 1083-9.

Liao, E. H., Hung, W., Abrams, B. and Zhen, M. (2004). An SCF-like ubiquitin ligase complex that controls presynaptic differentiation. Nature 430,345-50.

Litterman, N., Ikeuchi, Y., Gallardo, G., O'Connell, B. C., Sowa, M. E., Gygi, S. P., Harper, J. W. and Bonni, A. (2011). An OBSL1-Cul7Fbxw8 ubiquitin ligase signaling mechanism regulates Golgi morphology and dendrite patterning. PLoS Biology 9, e1001060.

Lowery, L. A. and Van Vactor, D. (2009). The trip of the tip: understanding the growth cone machinery. Nature Reviews Molecular Cell Biology 10, 332-43.

Mabb, A. M., Judson, M. C., Zylka, M. J. and Philpot, B. D. (2011). Angelman syndrome: insights into genomic imprinting and neurodevelopmental phenotypes. Trends in Neurosciences 34, 293-303.

Malatesta, P., Hartfuss, E. and Götz, M. (2000). Isolation of radial glial cells by fluorescentactivated cell sorting reveals a neuronal lineage. Development 127, 5253-63.

Mao, Y., Ge, X., Frank, C. L., Madison, J. M., Koehler, A. N., Doud, M. K., Tassa, C., Berry, E. M., Soda, T., Singh, K. K., Biechele, T., Petryshen, T. L., Moon, R. T., Haggarty, S. J. and Tsai, L.-h. (2009). Disrupted in schizophrenia 1 regulates neuronal progenitor proliferation via modulation of GSK3beta/beta-catenin signaling. Cell 136, $1017-31$.

Margolis, S. S., Salogiannis, J., Lipton, D. M., Mandel-Brehm, C., Wills, Z. P., Mardinly, A. R., Hu, L., Greer, P. L., Bikoff, J. B., Ho, H.-y. H., Soskis, M. J., Sahin, M. and Greenberg, M. E. (2010). EphB-mediated degradation of the RhoA GEF Ephexin5 relieves a developmental brake on excitatory synapse formation. Cell 143, 442-55.

Marin, O. (2003). Directional guidance of interneuron migration to the cerebral cortex relies on subcortical Slit1/2-independent repulsion and cortical attraction. Development 130, 1889-1901. 
Mehta, N., Loria, P. M. and Hobert, O. (2004). A genetic screen for neurite outgrowth mutants in Caenorhabditis elegans reveals a new function for the F-box ubiquitin ligase component LIN-23. Genetics 166, 1253-67.

Miller, J. J., Summers, M. K., Hansen, D. V., Nachury, M. V., Lehman, N. L., Loktev, A. and Jackson, P. K. (2006). Emi1 stably binds and inhibits the anaphase-promoting complex/cyclosome as a pseudosubstrate inhibitor. Genes \& Development 20, 2410-20.

Moore, D. L., Apara, A. and Goldberg, J. L. (2011). Krüppel-like transcription factors in the nervous system: novel players in neurite outgrowth and axon regeneration. Molecular and Cellular Neurosciences 47, 233-43.

Mori, D., Yamada, M., Mimori-Kiyosue, Y., Shirai, Y., Suzuki, A., Ohno, S., Saya, H., Wynshaw-Boris, A. and Hirotsune, S. (2009). An essential role of the aPKC-Aurora ANDEL1 pathway in neurite elongation by modulation of microtubule dynamics. Nature Cell Biology 11, 1057-68.

Morton, D. G., Roos, J. M. and Kemphues, K. J. (1992). par-4, a gene required for cytoplasmic localization and determination of specific cell types in Caenorhabditis elegans embryogenesis. Genetics 130, 771-90.

Morton, D. G., Shakes, D. C., Nugent, S., Dichoso, D., Wang, W., Golden, A. and Kemphues, K. J. (2002). The Caenorhabditis elegans par-5 gene encodes a 14-3-3 protein required for cellular asymmetry in the early embryo. Developmental Biology 241, 47-58.

Munro, E. M. (2006). PAR proteins and the cytoskeleton: a marriage of equals. Current Opinion in Cell Biology 18, 86-94.

Narayan, S., Tang, B., Head, S. R., Gilmartin, T. J., Sutcliffe, J. G., Dean, B. and Thomas, E. a. (2008). Molecular profiles of schizophrenia in the CNS at different stages of illness. Brain Research 1239, 235-48.

Nishimura, T., Kato, K., Yamaguchi, T., Fukata, Y., Ohno, S. and Kaibuchi, K. (2004). Role of the PAR-3-KIF3 complex in the establishment of neuronal polarity. Nature Cell Biology 6, 328-34.

Nishimura, T., Yamaguchi, T., Kato, K., Yoshizawa, M., Nabeshima, Y.-i., Ohno, S., Hoshino, M. and Kaibuchi, K. (2005). PAR-6-PAR-3 mediates Cdc42-induced Rac activation through the Rac GEFs STEF/Tiam1. Nature Cell Biology 7, 270-7.

Noctor, S. C., Flint, a. C., Weissman, T. a., Dammerman, R. S. and Kriegstein, a. R. (2001). Neurons derived from radial glial cells establish radial units in neocortex. Nature $409,714-20$. 
Noctor, S. C., Flint, A. C., Weissman, T. a., Wong, W. S., Clinton, B. K. and Kriegstein, A. R. (2002). Dividing precursor cells of the embryonic cortical ventricular zone have morphological and molecular characteristics of radial glia. The Journal of Neuroscience 22, 3161-73.

Nolan, M. E., Aranda, V., Lee, S., Lakshmi, B., Basu, S., Allred, D. C. and Muthuswamy, S. K. (2008). The polarity protein Par6 induces cell proliferation and is overexpressed in breast cancer. Cancer Research 68, 8201-9.

Ooi, L. and Wood, I. C. (2007). Chromatin crosstalk in development and disease: lessons from REST. Nature Reviews Genetics 8, 544-54.

Ouzir, M., Azorin, J. M., Adida, M., Boussaoud, D. and Battas, O. (2012). Insight in schizophrenia: from conceptualization to neuroscience. Psychiatry and Clinical Neurosciences $66,167-79$.

Ozdamar, B., Bose, R., Barrios-Rodiles, M., Wang, H.-R., Zhang, Y. and Wrana, J. L. (2005). Regulation of the polarity protein Par6 by TGFbeta receptors controls epithelial cell plasticity. Science 30\%, 1603-9.

Ozdinler, P. H. and Macklis, J. D. (2006). IGF-I specifically enhances axon outgrowth of corticospinal motor neurons. Nature Neuroscience 9, 1371-81.

Paisán-Ruiz, C., Guevara, R., Federoff, M., Hanagasi, H., Sina, F., Elahi, E., Schneider, S. a., Schwingenschuh, P., Bajaj, N., Emre, M., Singleton, A. B., Hardy, J., Bhatia, K. P., Brandner, S., Lees, A. J. and Houlden, H. (2010). Early-onset L-dopa-responsive parkinsonism with pyramidal signs due to ATP13A2, PLA2G6, FBXO7 and spatacsin mutations. Movement Disorders 25, 1791-800.

Peters, J. M. (1998). SCF and APC: the Yin and Yang of cell cycle regulated proteolysis. Current Opinion in Cell Biology 10, 759-68.

Peters, J.-M. (2002). The anaphase-promoting complex: proteolysis in mitosis and beyond. Molecular Cell 9, 931-43.

Peters, J.-M. (2006). The anaphase promoting complex/cyclosome: a machine designed to destroy. Nature Reviews Molecular Cell Biology 7, 644-56.

Pickart, C. M. and Eddins, M. J. (2004). Ubiquitin: structures, functions, mechanisms. Biochimica et Biophysica Acta 1695, 55-72.

Pierfelice, T., Alberi, L. and Gaiano, N. (2011). Notch in the vertebrate nervous system: an old dog with new tricks. Neuron $69,840-55$. 
Polleux, F. and Snider, W. (2010). Initiating and growing an axon. Cold Spring Harbor Perspectives in Biology 2, a001925.

Puram, S. V., Kim, A. H., Ikeuchi, Y., Wilson-Grady, J. T., Merdes, A., Gygi, S. P. and Bonni, A. (2011). A CaMKII $\beta$ signaling pathway at the centrosome regulates dendrite patterning in the brain. Nature Neuroscience 14, 973-83.

Qing, H., Zhou, W., Christensen, M. a., Sun, X., Tong, Y. and Song, W. (2004). Degradation of BACE by the ubiquitin-proteasome pathway. FASEB Journal 18, 1571-3.

Saiga, T., Fukuda, T., Matsumoto, M., Tada, H., Okano, H. J., Okano, H. and Nakayama, K. I. (2009). Fbxo45 forms a novel ubiquitin ligase complex and is required for neuronal development. Molecular and Cellular Biology 29, 3529-43.

Santra, M. K., Wajapeyee, N. and Green, M. R. (2009). F-box protein FBXO31 mediates cyclin D1 degradation to induce G1 arrest after DNA damage. Nature 459, 722-5.

Sasai, Y., Lu, B., Steinbeisser, H. and De Robertis, E. M. (1995). Regulation of neural induction by the Chd and Bmp-4 antagonistic patterning signals in Xenopus. Nature $376,333-6$.

Schwamborn, J. C., Berezikov, E. and Knoblich, J. A. (2009). The TRIM-NHL protein TRIM32 activates microRNAs and prevents self-renewal in mouse neural progenitors. Cell 136, 913-25.

Schwamborn, J. C., Khazaei, M. R. and Püschel, A. W. (2007). The interaction of mPar3 with the ubiquitin ligase Smurf2 is required for the establishment of neuronal polarity. The Journal of Biological Chemistry 282, 35259-68.

Shi, S.-h., Cheng, T., Jan, L. Y. and Jan, Y.-n. (2004). APC and GSK-3beta are involved in mPar3 targeting to the nascent axon and establishment of neuronal polarity. Current Biology 14, 2025-32.

Shi, S.-h., Jan, L. Y. and Jan, Y.-n. (2003). Hippocampal neuronal polarity specified by spatially localized mPar3/mPar6 and PI 3-kinase activity. Cell 112, 63-75.

Shin, K., Fogg, V. C. and Margolis, B. (2006). Tight junctions and cell polarity. Annual Review of Cell and Developmental Biology 22, 207-35.

Shirogane, T., Jin, J., Ang, X. L. and Harper, J. W. (2005). SCFbeta-TRCP controls clockdependent transcription via casein kinase 1-dependent degradation of the mammalian period-1 (Per1) protein. The Journal of Biological Chemistry 280, 26863-72.

Silies, M. and Klämbt, C. (2010). APC/C(Fzr/Cdh1)-dependent regulation of cell adhesion controls glial migration in the Drosophila PNS. Nature Neuroscience 13, 1357-64. 
Solecki, D. J. (2012). Sticky situations: recent advances in control of cell adhesion during neuronal migration. Current Opinion in Neurobiology 22, 1-8.

Solecki, D. J., Model, L., Gaetz, J., Kapoor, T. M. and Hatten, M. E. (2004). Par6alpha signaling controls glial-guided neuronal migration. Nature Neuroscience 7, 1195-203.

Solecki, D. J., Trivedi, N., Govek, E.-E., Kerekes, R. a., Gleason, S. S. and Hatten, M. E. (2009). Myosin II motors and F-actin dynamics drive the coordinated movement of the centrosome and soma during CNS glial-guided neuronal migration. Neuron 63, 63-80.

Soriano, E. and Del Río, J. A. (2005). The cells of cajal-retzius: still a mystery one century after. Neuron 46, 389-94.

Spence, J., Sadis, S., Haas, A. L. and Finley, D. (1995). A ubiquitin mutant with specific defects in DNA repair and multiubiquitination. Molecular and Cellular Biology 15, $1265-73$.

Stegmüller, J., Huynh, M. A., Yuan, Z., Konishi, Y. and Bonni, A. (2008). TGFbetaSmad2 signaling regulates the Cdh1-APC/SnoN pathway of axonal morphogenesis. The Journal of Neuroscience 28, 1961-9.

Stegmüller, J., Konishi, Y., Huynh, M. A., Yuan, Z., Dibacco, S. and Bonni, A. (2006). Cell-intrinsic regulation of axonal morphogenesis by the Cdh1-APC target SnoN. Neuron $50,389-400$.

Streit, A. and Stern, C. D. (1999). Neural induction. A bird's eye view. Trends in Genetics $15,20-4$.

Sütterlin, C. and Colanzi, A. (2010). The Golgi and the centrosome: building a functional partnership. The Journal of Cell Biology 188, 621-8.

Tabuse, Y., Izumi, Y., Piano, F., Kemphues, K. J., Miwa, J. and Ohno, S. (1998). Atypical protein kinase $\mathrm{C}$ cooperates with PAR-3 to establish embryonic polarity in Caenorhabditis elegans. Development 125, 3607-14.

Tada, H., Okano, H. J., Takagi, H., Shibata, S., Yao, I., Matsumoto, M., Saiga, T., Nakayama, K. I., Kashima, H., Takahashi, T., Setou, M. and Okano, H. (2010). Fbxo45, a novel ubiquitin ligase, regulates synaptic activity. The Journal of Biological Chemistry 285, 3840-9.

Tanaka, T., Serneo, F. F., Higgins, C., Gambello, M. J., Wynshaw-Boris, A. and Gleeson, J. G. (2004). Lis1 and doublecortin function with dynein to mediate coupling of the nucleus to the centrosome in neuronal migration. The Journal of Cell Biology 165, $709-21$. 
Tang, Z., Li, B., Bharadwaj, R., Zhu, H., Ozkan, E., Hakala, K., Deisenhofer, J. and $\mathrm{Yu}, \mathrm{H}$. (2001). APC2 Cullin protein and APC11 RING protein comprise the minimal ubiquitin ligase module of the anaphase-promoting complex. Molecular Biology of the Cell 12, 3839-51.

Tessier-Lavigne, M. and Goodman, C. S. (1996). The molecular biology of axon guidance. Science 274, 1123-33.

Tsvetkov, L. M., Yeh, K. H., Lee, S. J., Sun, H. and Zhang, H. (1999). p27(Kip1) ubiquitination and degradation is regulated by the $\mathrm{SCF}(\mathrm{Skp} 2)$ complex through phosphorylated Thr187 in p27. Current Biology 9, 661-4.

van Roessel, P., Elliott, D. A., Robinson, I. M., Prokop, A. and Brand, A. H. (2004). Independent regulation of synaptic size and activity by the anaphase-promoting complex. Cell 119, 707-18.

Vohra, B. P. S., Fu, M. and Heuckeroth, R. O. (2007). Protein kinase Czeta and glycogen synthase kinase-3beta control neuronal polarity in developing rodent enteric neurons, whereas SMAD specific E3 ubiquitin protein ligase 1 promotes neurite growth but does not influence polarity. The Journal of Neuroscience 27, 9458-68.

Wang, H.-r., Zhang, Y., Ozdamar, B., Ogunjimi, A. A., Alexandrova, E., Thomsen, G. H. and Wrana, J. L. (2003). Regulation of cell polarity and protrusion formation by targeting RhoA for degradation. Science 302, 1775-9.

Wang, X., Trotman, L. C., Koppie, T., Alimonti, A., Chen, Z., Gao, Z., Wang, J., Erdjument-Bromage, H., Tempst, P., Cordon-Cardo, C., Pandolfi, P. P. and Jiang, X. (2007). NEDD4-1 is a proto-oncogenic ubiquitin ligase for PTEN. Cell 128, 129-39.

Watanabe, T., von der Kammer, H., Wang, X., Shintani, Y. and Horiguchi, T. (2012). Neuronal Expression of F-Box and Leucine-Rich-Repeat Protein 2 Decreases over Braak Stages in the Brains of Alzheimer's Disease Patients. Neurodegenerative diseases 000, $1-10$.

Watts, J. L., Etemad-Moghadam, B., Guo, S., Boyd, L., Draper, B. W., Mello, C. C., Priess, J. R. and Kemphues, K. J. (1996). par-6, a gene involved in the establishment of asymmetry in early C. elegans embryos, mediates the asymmetric localization of PAR-3. Development 122, 3133-40.

Welcker, M. and Clurman, B. E. (2008). FBW7 ubiquitin ligase: a tumour suppressor at the crossroads of cell division, growth and differentiation. Nature Reviews Cancer 8 , 83-93. 
Westbrook, T. F., Hu, G., Ang, X. L., Mulligan, P., Pavlova, N. N., Liang, A., Leng, Y., Maehr, R., Shi, Y., Harper, J. W. and Elledge, S. J. (2008). SCFbeta-TRCP controls oncogenic transformation and neural differentiation through REST degradation. Nature 452, 370-4.

Wodarz, A. (2002). Establishing cell polarity in development. Nature Cell Biology 4, E39-44.

Yang, Y., Kim, A. H. and Bonni, A. (2010). The dynamic ubiquitin ligase duo: Cdh1-APC and Cdc20-APC regulate neuronal morphogenesis and connectivity. Current Opinion in Neurobiology 20, 92-9.

Yang, Y., Kim, A. H., Yamada, T., Wu, B., Bilimoria, P. M., Ikeuchi, Y., de la Iglesia, N., Shen, J. and Bonni, A. (2009). A Cdc20-APC ubiquitin signaling pathway regulates presynaptic differentiation. Science 326, 575-8.

Yao, I., Takagi, H., Ageta, H., Kahyo, T., Sato, S., Hatanaka, K., Fukuda, Y., Chiba, T., Morone, N., Yuasa, S., Inokuchi, K., Ohtsuka, T., Macgregor, G. R., Tanaka, K. and Setou, M. (2007). SCRAPPER-dependent ubiquitination of active zone protein RIM1 regulates synaptic vesicle release. Cell 130, 943-57.

Yi, J. J., Barnes, A. P., Hand, R., Polleux, F. and Ehlers, M. D. (2010). TGF-beta signaling specifies axons during brain development. Cell 142, 144-57.

Zhang, H. and Macara, I. G. (2006). The polarity protein PAR-3 and TIAM1 cooperate in dendritic spine morphogenesis. Nature Cell Biology 8, 227-37.

Zhang, H. and Macara, I. G. (2008). The PAR-6 polarity protein regulates dendritic spine morphogenesis through p190 RhoGAP and the Rho GTPase. Developmental Cell 14, $216-26$.

Zhang, J., Li, H., Zhou, T., Zhou, J. and Herrup, K. (2012). Cdk5 Levels Oscillate during the Neuronal Cell Cycle: Cdh1 UBIQUITINATION TRIGGERS PROTEOSOMEDEPENDENT DEGRADATION DURING S-PHASE. The Journal of Biological Chemistry $28 \%, 25985-94$.

Zhang, L. I. and Poo, M. M. (2001). Electrical activity and development of neural circuits. Nature Neuroscience 4, 1207-14.

Zhang, X., Zhu, J., Yang, G.-y., Wang, Q.-J., Qian, L., Chen, Y.-M., Chen, F., Tao, Y., Hu, H.-s., Wang, T. and Luo, Z.-g. (2007). Dishevelled promotes axon differentiation by regulating atypical protein kinase C. Nature Cell Biology 9, 743-54.

Zhao, J., Ren, Y., Jiang, Q. and Feng, J. (2003). Parkin is recruited to the centrosome in response to inhibition of proteasomes. Journal of Cell Science 116, 4011-9. 
Zheng, N., Schulman, B. a., Song, L., Miller, J. J., Jeffrey, P. D., Wang, P., Chu, C., Koepp, D. M., Elledge, S. J., Pagano, M., Conaway, R. C., Conaway, J. W., Harper, J. W. and Pavletich, N. P. (2002). Structure of the Cul1-Rbx1-Skp1-F boxSkp2 SCF ubiquitin ligase complex. Nature 416, 703-9.

Zhou, F.-q. and Snider, W. D. (2006). Intracellular control of developmental and regenerative axon growth. Philosophical Transactions of the Royal Society of London. Series B, Biological Sciences 361, 1575-92.

Zhou, Y., Ching, Y.-P., Chun, A. C. S. and Jin, D.-Y. (2003). Nuclear localization of the cell cycle regulator $\mathrm{CDH} 1$ and its regulation by phosphorylation. The Journal of Biological Chemistry 278, 12530-6.

Zmuda, J. F. and Rivas, R. J. (1998). The Golgi apparatus and the centrosome are localized to the sites of newly emerging axons in cerebellar granule neurons in vitro. Cell Motility and the Cytoskeleton 41, 18-38. 


\section{A. Appendix}

Table A.1: List of plasmids used in the study

\begin{tabular}{ll}
\hline Plasmid & Reference \\
\hline pCMVmyc/FBXO31 WT and $\Delta \mathrm{F}$ & $\begin{array}{l}\text { Dr. Raman Kumar and Prof. David } \\
\text { Callen, University of Adelaide, Australia }\end{array}$ \\
C2-GFP/FBXO31 WT and $\Delta \mathrm{F}$ & \\
pCMVmyc/FBXO31 DBM1-6 & \\
\hline pHACE/PKC $\iota$ & Addgene \\
pHACE/PKC $\zeta$ & \\
pKmyc/Par6c & \\
pKmyc/Par3b & Dr. Judith Stegmüller \\
pCMVmyc/Skp1 & \\
Bcl-xL & \\
pCDNA3/Flag-Cdh1 & \\
C1-GFP/Cdh1 & \\
pBS-U6/Cdh1 RNAi & \\
\hline C2-GFP/FBXO31 $\Delta 1-\Delta 5$ & \\
pBS-U6/FBXO31 RNAi \#1, \#2, \#3 & \\
pBS-U6/FBXO31 RNAi \#1/CMVGFP & \\
pCMVmyc/FBXO31-Res & \\
pCDNA3/Flag-FBXO31 & \\
pCMVmyc/Par6c & \\
pCMVmyc/Par6b & \\
pCMVmyc/Renilla-CCND1 & \\
pCMVmyc/Renilla-Par6c & \\
pBS-U6/Par6c RNAi & \\
pCMVmyc/Par6c-Res & \\
pCMVmyc/Par6c deletion mutants & \\
pCMVmyc/FBXO31 DBM F1, F2 & \\
\hline
\end{tabular}


Table A.2: List of primers used for FBX gene expression study: $\mathrm{S}$ (sense), as (anti-sense)

\begin{tabular}{|c|c|c|c|}
\hline $\begin{array}{l}\text { AGCT } \\
\text { no. }\end{array}$ & $\begin{array}{l}\text { Sense/ } \\
\text { anti- } \\
\text { sense }\end{array}$ & Sequence (5'-3') & $\begin{array}{l}\text { PCR } \\
\text { primer } \\
\text { for }\end{array}$ \\
\hline 14352 & $\mathrm{~s}$ & GTACTCGGGTCGCACTGATG & FBXO2 \\
\hline 14353 & as & ACAATGCTAGGAGCGCGGGA & FBXO2 \\
\hline 14354 & $\mathrm{~s}$ & GCCATCCCCCGATTCCACAT & FBXO3 \\
\hline 14355 & as & GCACAGTCAGTCCTTCCTGC & FBXO3 \\
\hline 14356 & $\mathrm{~s}$ & CCATGTGGGACTGCAGCTGT & FBXO9 \\
\hline 14357 & as & CTCTCCAGTGTGCTCCCTGA & FBXO9 \\
\hline 14358 & $\mathrm{~s}$ & CCAGCTACCCCATGCATGAC & FBXO11 \\
\hline 14359 & as & CCCGAGCTCTGACATCCTGA & FBXO11 \\
\hline 14360 & $\mathrm{~s}$ & GTGTGGGGCAGTGCAACAAC & FBXO18 \\
\hline 14361 & as & GTTCCCTGGCCTGAGGCATT & FBXO18 \\
\hline 14362 & $\mathrm{~s}$ & AATCTGGATTCCCAGCGGCC & FBXO20 \\
\hline 14363 & as & ATCACATGGCGGTTGGCCGT & FBXO20 \\
\hline 14364 & $\mathrm{~s}$ & TGTGCACAGCCTGCCTCATG & FBXO21 \\
\hline 14365 & as & GCCTGACTCAGCGACCTTGT & FBXO21 \\
\hline 14366 & $\mathrm{~s}$ & ATCTTGGCTGGAGGCCAGGT & FBXO22 \\
\hline 14367 & as & AAAGGTGCTCTCAGCGAGGC & FBXO22 \\
\hline 14368 & $\mathrm{~s}$ & ACACGCTACACTTCTGCCGC & FBXO25 \\
\hline 14369 & as & CACAGCCACTGGGGTTGTGA & FBXO25 \\
\hline 14370 & $\mathrm{~s}$ & TCACGAGGTGCTGCAGGAGT & FBXO28 \\
\hline 14371 & as & GCTTCCAGGCGATCAGACAC & FBXO28 \\
\hline 14372 & $\mathrm{~s}$ & ATGGGCCACCTGCTAAGGAC & FBXO31 \\
\hline 14373 & as & AAGTGCATGCTTCCCCCACC & FBXO31 \\
\hline 14374 & $\mathrm{~s}$ & TGTATGCGGCTGCAGGTCCT & FBXO41 \\
\hline 14375 & as & GGATTCCTAGGCTCGCCTAG & FBXO41 \\
\hline 14376 & $\mathrm{~s}$ & TGCGTCCAACTCCTGTCGTC & FBXO44 \\
\hline 14377 & as & TGTCTGTCCCTGCTCTCCAG & FBXO44 \\
\hline 14378 & $\mathrm{~s}$ & TCAGCCCTGGGTCTGTACCA & FBXW2 \\
\hline 14379 & as & GGTTCATGCAGTTGGCTGCC & FBXW2 \\
\hline 14380 & $\mathrm{~s}$ & GCATGGGCCTGTCTCCTGAT & FBXW5 \\
\hline 14381 & as & GTGGAGCCTGCAGAACACGA & FBXW5 \\
\hline 14382 & $\mathrm{~s}$ & CTGGGATGTGGAGACAGGGA & FBXW7 \\
\hline 14383 & as & ACTCCCGACTGCACACACCA & FBXW7 \\
\hline 14384 & $\mathrm{~s}$ & GCTGGCAGGCCTTGACATCA & FBXL11 \\
\hline 14385 & as & GGGAATGTGTCGTTGGCCTC & FBXL11 \\
\hline 14386 & $\mathrm{~s}$ & ACTGAGAGTCGAGGCCGACT & FBXL19 \\
\hline
\end{tabular}




\begin{tabular}{llll}
14387 & as & CCTCCCAACAGGGGGTGAAA & FBXL19 \\
14388 & s & GATAGACGACACCCCAGTCG & FBXL3 \\
14389 & as & TCATCAAGAGGCCGCAACCC & FBXL3 \\
14390 & s & GACTGTAACTGGTGCAGGGC & FBXL5 \\
14391 & as & CAGCAGTGGAAGCTGGTGTC & FBXL5 \\
\hline
\end{tabular}




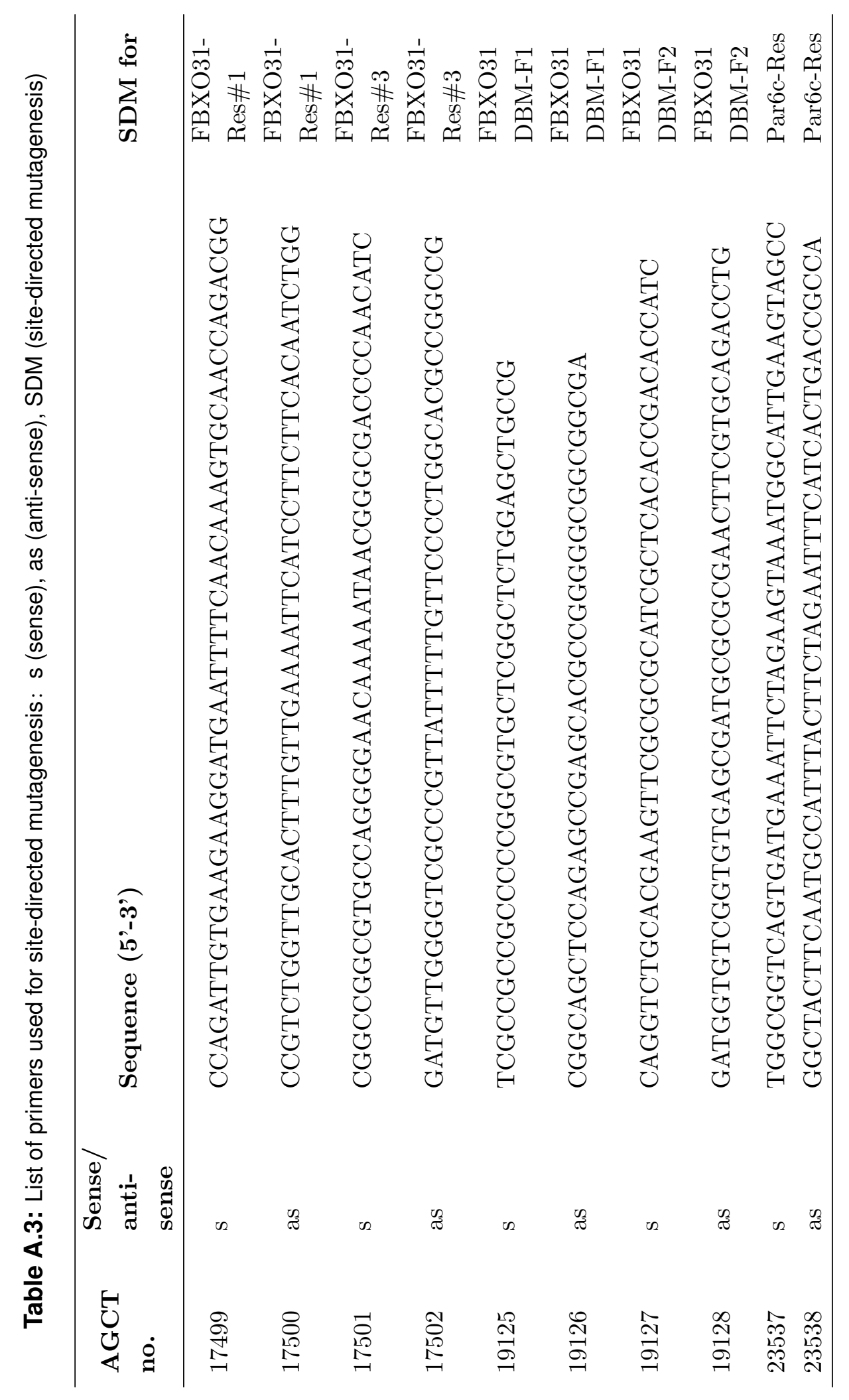




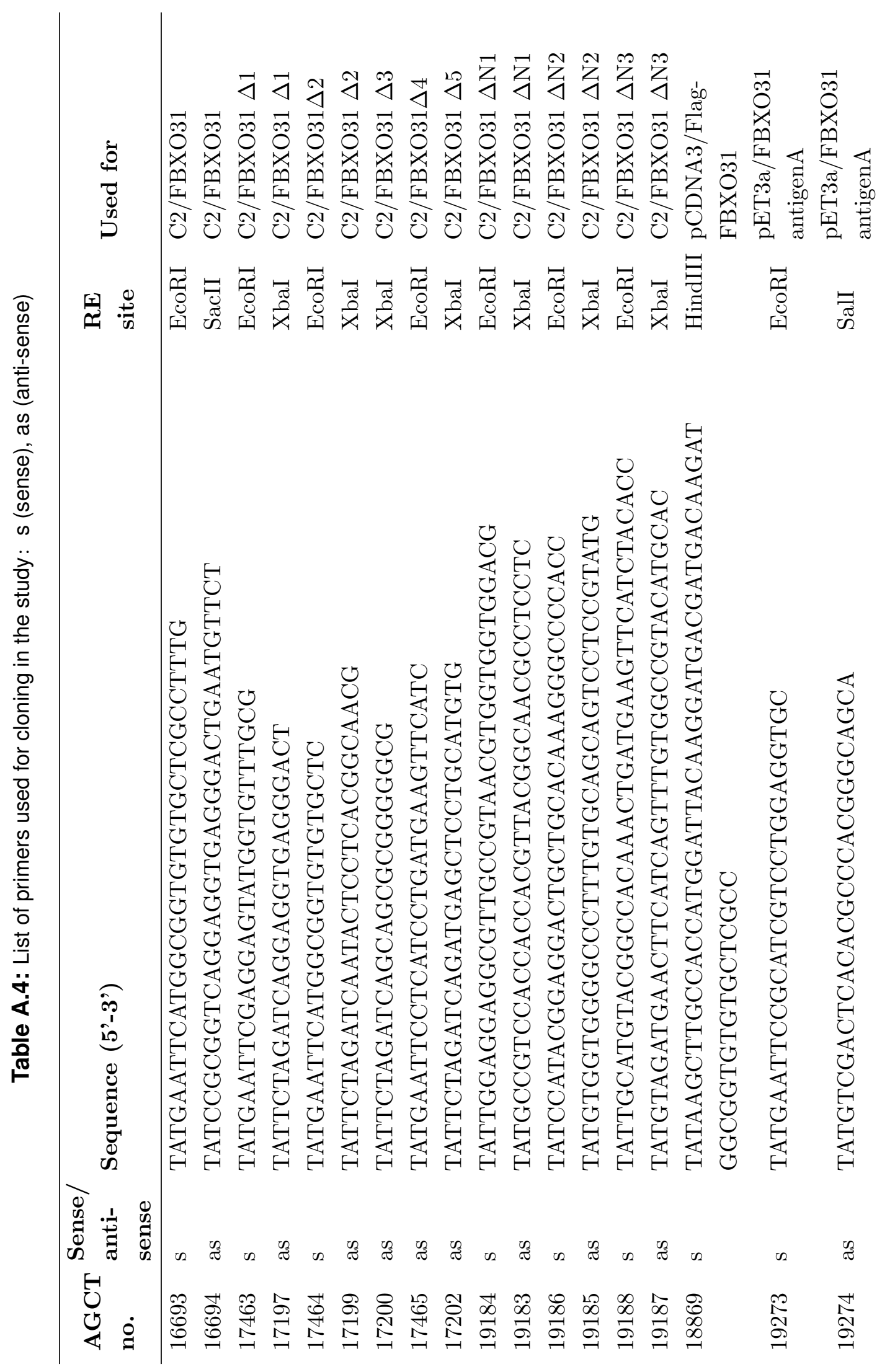




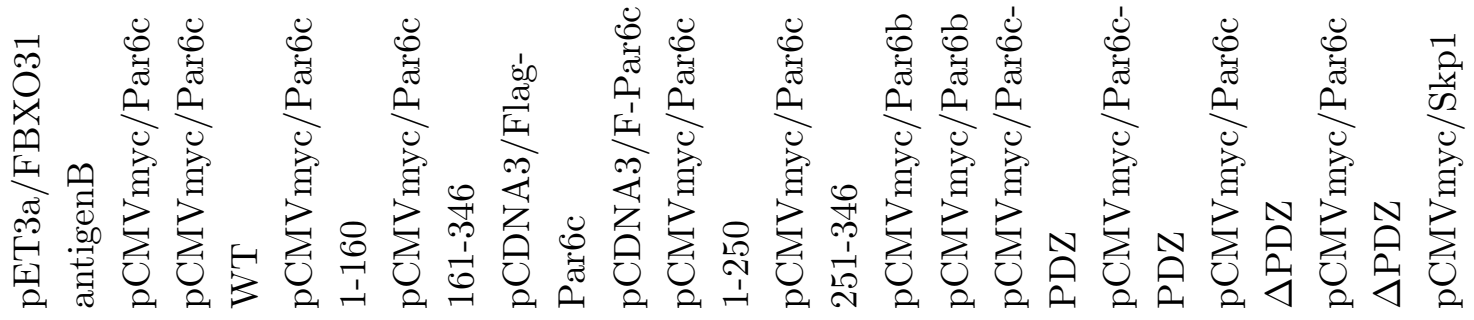

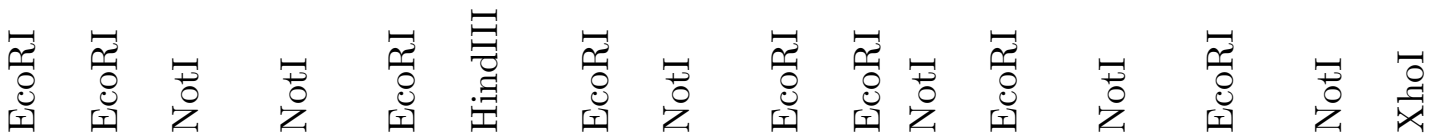

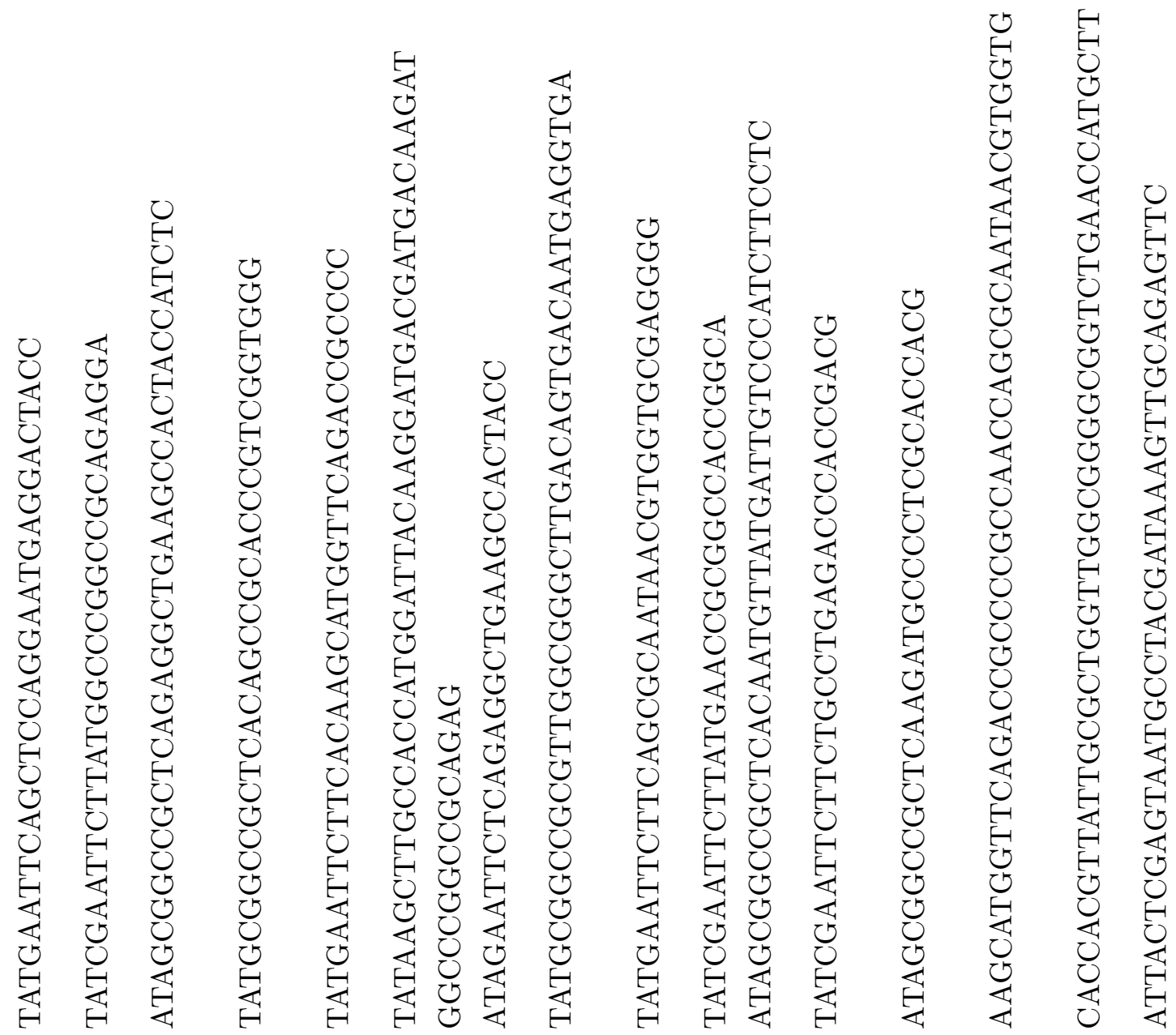

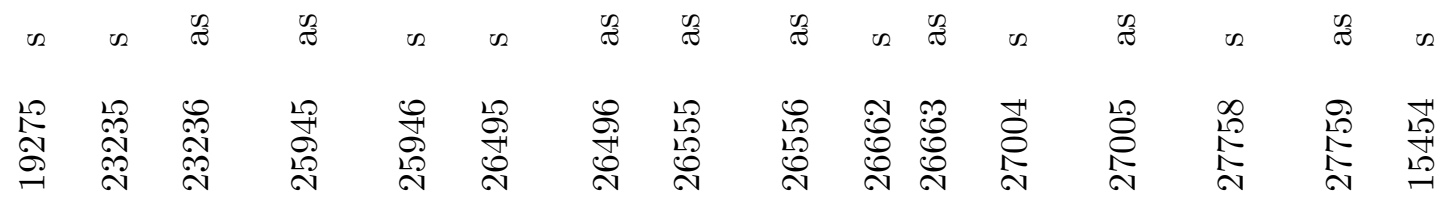




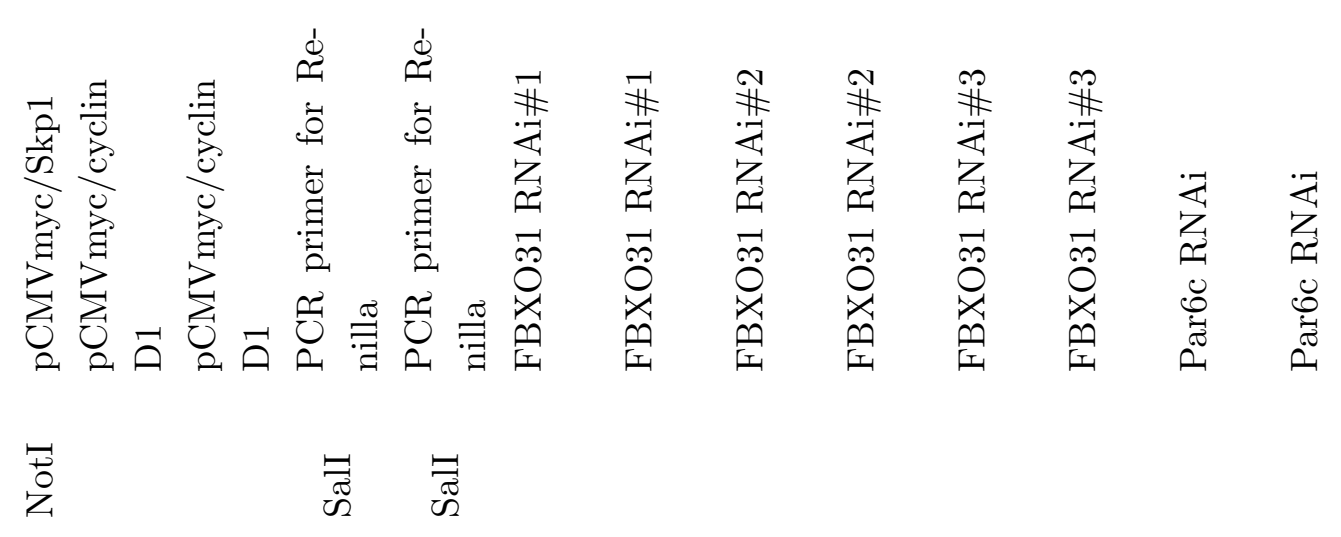

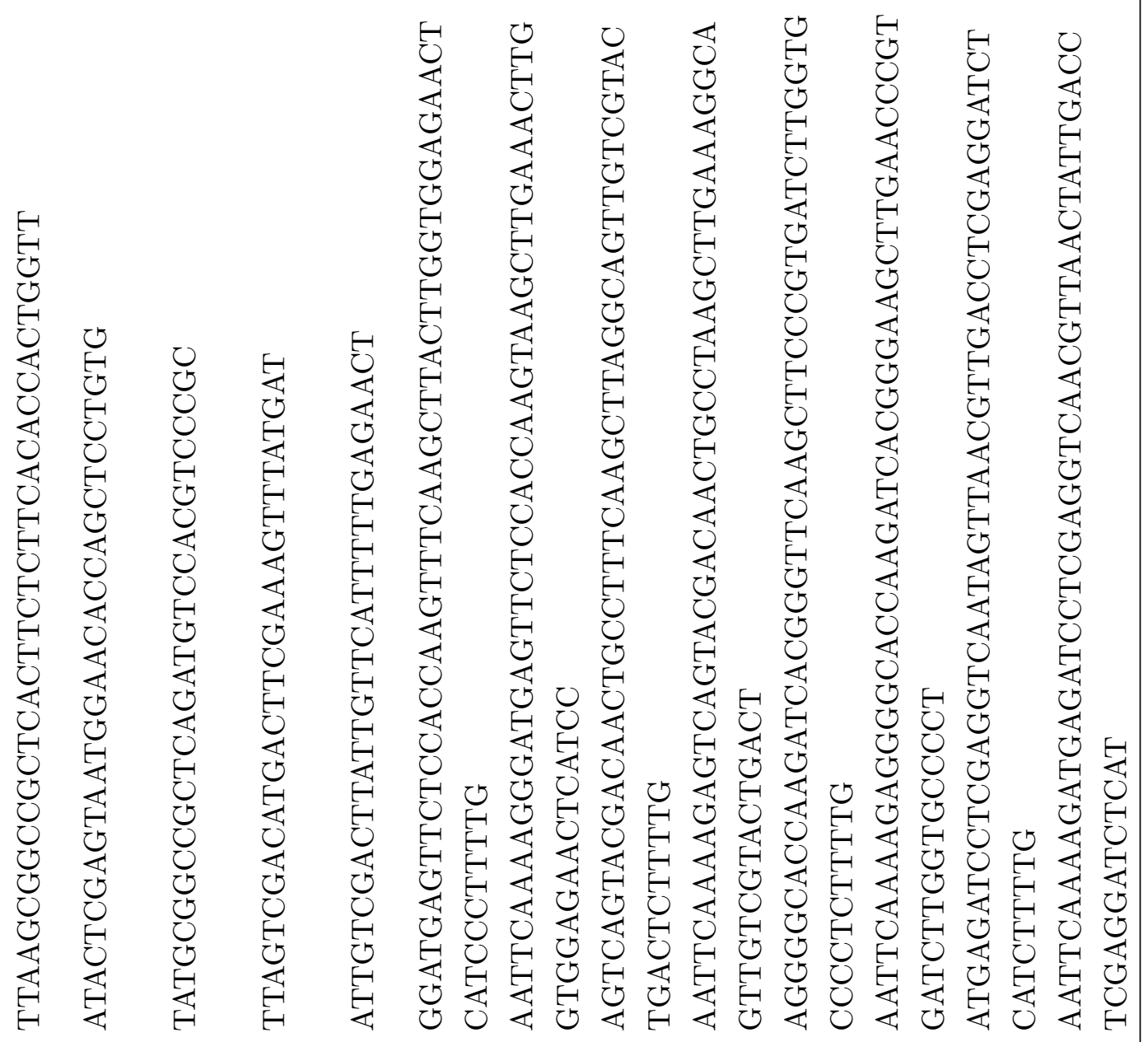

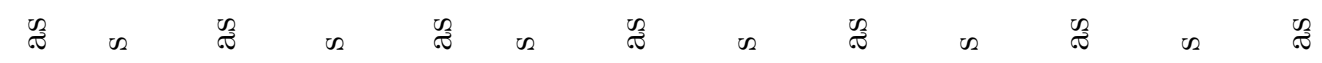

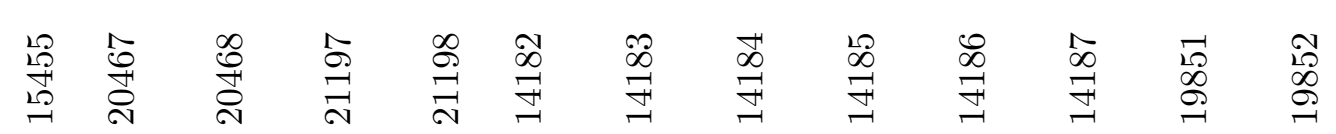




\title{
Curriculum Vitae
}

\author{
MAYUR VADHVANI
}

born on $26^{\text {th }}$ October, 1982 Katihar, India

Hermann-Rein-Str. 3, 37075, Göttingen, Germany

Phone +49 (0) 5513899564

vadhvani@em.mpg.de

\section{Education}

Oct 2007 - present

Aug 2004 - Jul 2007

July 2001 - May 2004

\section{Teaching}

Sep 2011 - Dec 2011

Feb 2010 - Sep 2010

Dec 2010

Sep 2006

\section{Scholarships \& Prizes}

Sep 2008 - Aug 2009

Oct 2007- Aug 2008

Aug 2004- July 2007
International MSc/PhD IMPRS Neuroscience program, Ph.D. thesis: The role of E3 ubiquitin ligase FBXO31-SCF in neuronal morphogenesis, MPI of Experimental Medicine, Göttingen, Germany

M.Sc. thesis: Structural plasticity in the adult mammalian brain: Molecular and cellular mechanisms, TATA Institute of Fundamental Research (TIFR), Mumbai, India

B.Sc.(Hons.) Department of Biomedical Sciences, Acharya Narendra Dev College, University of Delhi, Delhi, India

S. Krishnan for Master's thesis entitled 'The crosstalk between Cdh1-APC and FBXO31-SCF E3 ubiquitin ligases'

David Brockelt for Bachelor's thesis entitled 'Functional characterization of interaction of DISC1 and FBXO31 proteins' Tutor for method course on 'Immunocytochemistry of cultured cerebellar granule neurons'

Teaching assistant for International Brain Research Organization (IBRO) Neuroscience School held at TIFR, Mumbai, India.

Max Planck Research Stipend

Stipend of the Excellence Foundation for the Promotion of the Max Planck Society

Junior Research Fellowship funded by the Department of Biotechnology (DBT), India 


\section{Research Experience}

Sep 2008 - present

Ph.D. thesis: The role of E3 ubiquitin ligase FBXO31-SCF in neuronal morphogenesis, supervisor: Dr. Judith Stegmüller, MPI of Experimental Medicine, Göttingen, Germany.

May 2008 - Jun 2008 Lab rotation project: Characterization of FBXO31 protein in the central nervous system, supervisor: Dr. Judith Stegmüller, MPI of Experimental Medicine, Göttingen, Germany.

Mar 2008 - Apr 2008 Lab rotation project: Examining neurogenesis in the adult mammalian cortex, supervisor: Prof. G. Flügge, German Primate Center, Göttingen.

Jan 2008 - Feb 2008 Lab rotation project: Therapeutic approaches for cerebral ischemia: potential use of adult neural stem cells in mice, supervisor: Prof. Mathias Bähr at Universitätsmedizin Göttingen.

Aug 2004 - July 2007 MSc thesis: Structural plasticity in the adult mammalian brain: Molecular and cellular mechanisms, supervisor: Dr. Vidita Vaidya, TIFR, Mumbai, India.

May 2003 - July 2003 Summer research project: Expression and isolation of chimeric recombinant antibody against human chorionic gonadotropin ( $h C G$ ) in various plant species, supervisor: Prof. G.P. Talwar, Talwar Research Foundation, New Delhi, India.

\section{Conferences}

May 2011

From molecules to mind: Making sense of the brain - Neurizons 2011, Göttingen, Germany (poster)

Sept 2009

Molecular Neurobiology: Pathways in Health and Disease $-5^{\text {th }}$

Westerberger Herbsttagung, Osnabrück, Germany (poster)

May 2008 $6^{\text {th }}$ Life Science Day, Weizmann Institute, Rehovot, Israel

\section{Extra-curricular activities}

Sep 2006

Student co-organizer and assistant for International Brain Research Organization (IBRO) Neuroscience School, TIFR, Mumbai, India

Jan 2005 Student co-organizer for $30^{\text {th }}$ Mahabaleshwar Seminar 2004 on Evolution of Developmental Mechanisms, Mahabaleshwar, India

Oct 2003

Student volunteer for National symposium - Biotechnology: Expanding Horizons, Indian National Science Academy, New Delhi, India 